Supporting Information

\title{
4,5-Dioxo-imidazolinium Cation Activation of 1-Acyl-1-carbamoyl \\ Oximes: Access to Cyanoformamides Using Dichloroimidazolidinedione
}

Yu Gao, ${ }^{\dagger}$ Zhihao Zhang, ${ }^{\dagger}$ Zhenjiang Li, ${ }^{*},{ }^{+},+$Tianfo Guo, ${ }^{+}$Yuejia Zhu, ${ }^{\dagger}$ Zhiwei Yao, ${ }^{\dagger}$ Bo Liu, ${ }^{\dagger}$ Yongqiang

$$
\mathrm{Li}^{\dagger}{ }^{\dagger} \text { and Kai Guo*,+, }
$$

${ }^{\dagger}$ College of Biotechnology and Pharmaceutical Engineering, Nanjing Tech University, 30 Puzhu Road South, Nanjing 211816, P. R. China

₹State Key Laboratory of Materials-Oriented Chemical Engineering, Nanjing Tech University, 30

Puzhu Road South, Nanjing 211816, P. R. China

E-mail: zjli@njtech.edu.cn, guok@njtech.edu.cn

\section{Table of Contents}

Copies of ${ }^{1} \mathrm{H}$ and ${ }^{13} \mathrm{C}$ NMR of New Starting Materials S2-S12

Copies of ${ }^{1} \mathrm{H}$ and ${ }^{13} \mathrm{C}$ NMR of Products 3a-3af..... S13-S45

Copies of ${ }^{1} \mathrm{H}$ and ${ }^{13} \mathrm{C}$ NMR of Compounds 4-7 S46-S49

Copies of ${ }^{1} \mathrm{H}$ and ${ }^{13} \mathrm{C}$ NMR of $\boldsymbol{N}$-Phenylacetamide S50 


\section{NMR Spectra of Compounds}

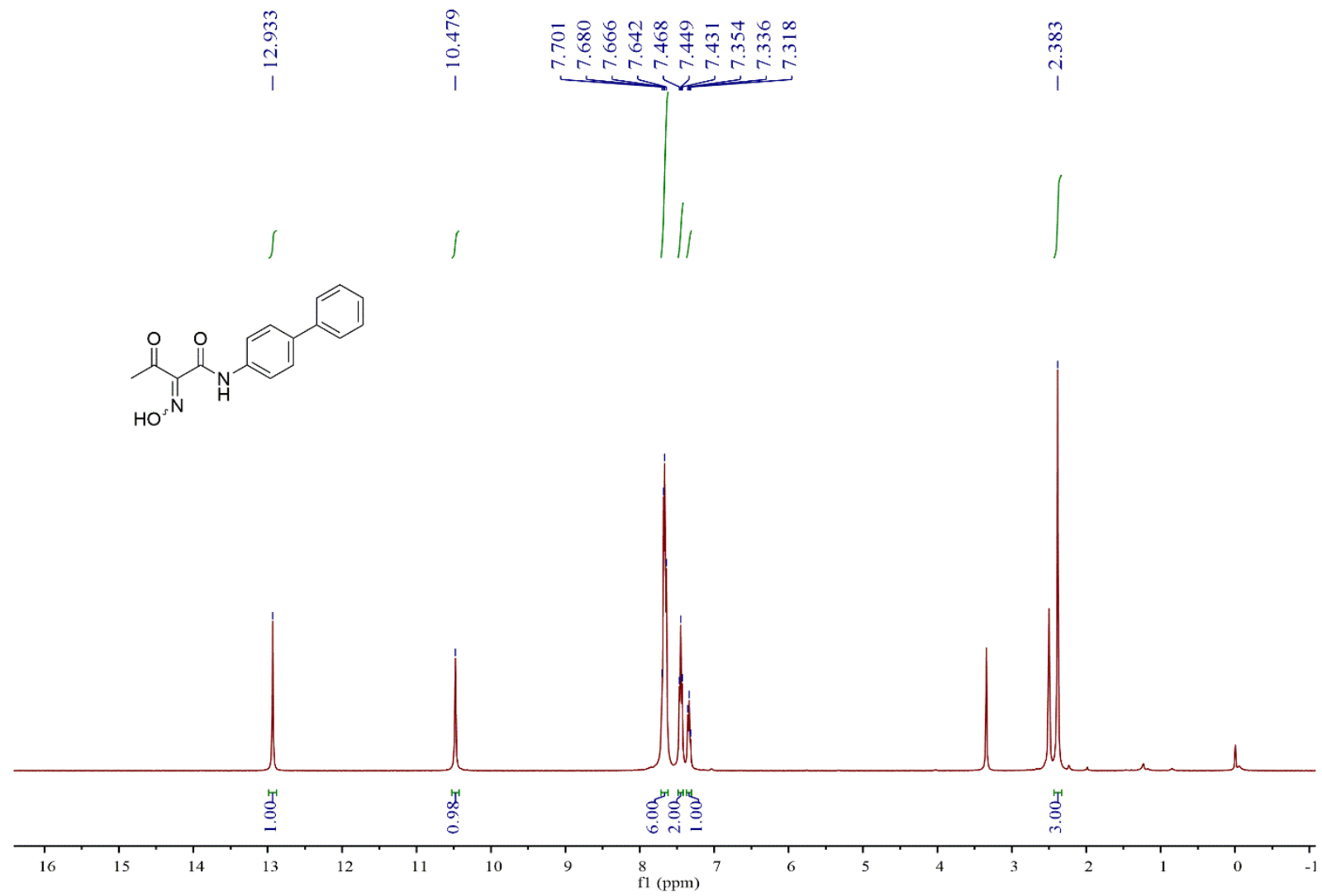

${ }^{1} \mathrm{H}$ NMR Spectrum of Compound $2 \mathbf{k}\left(400 \mathrm{MHz},\left(\mathrm{CD}_{3}\right)_{2} \mathrm{SO}\right)$
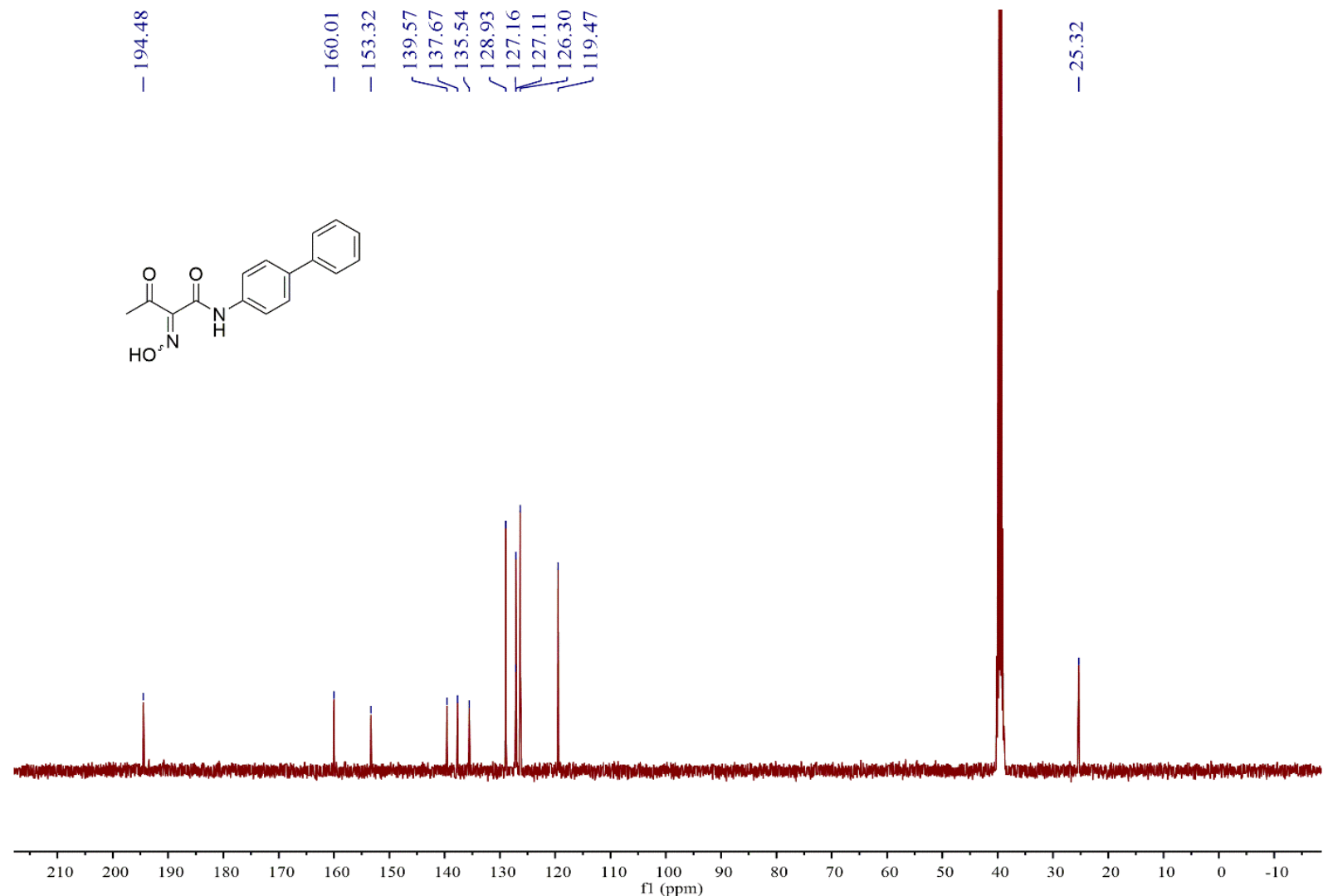

${ }^{13} \mathrm{C}$ NMR Spectrum of Compound $2 \mathbf{k}\left(100 \mathrm{MHz},\left(\mathrm{CD}_{3}\right)_{2} \mathrm{SO}\right)$ 


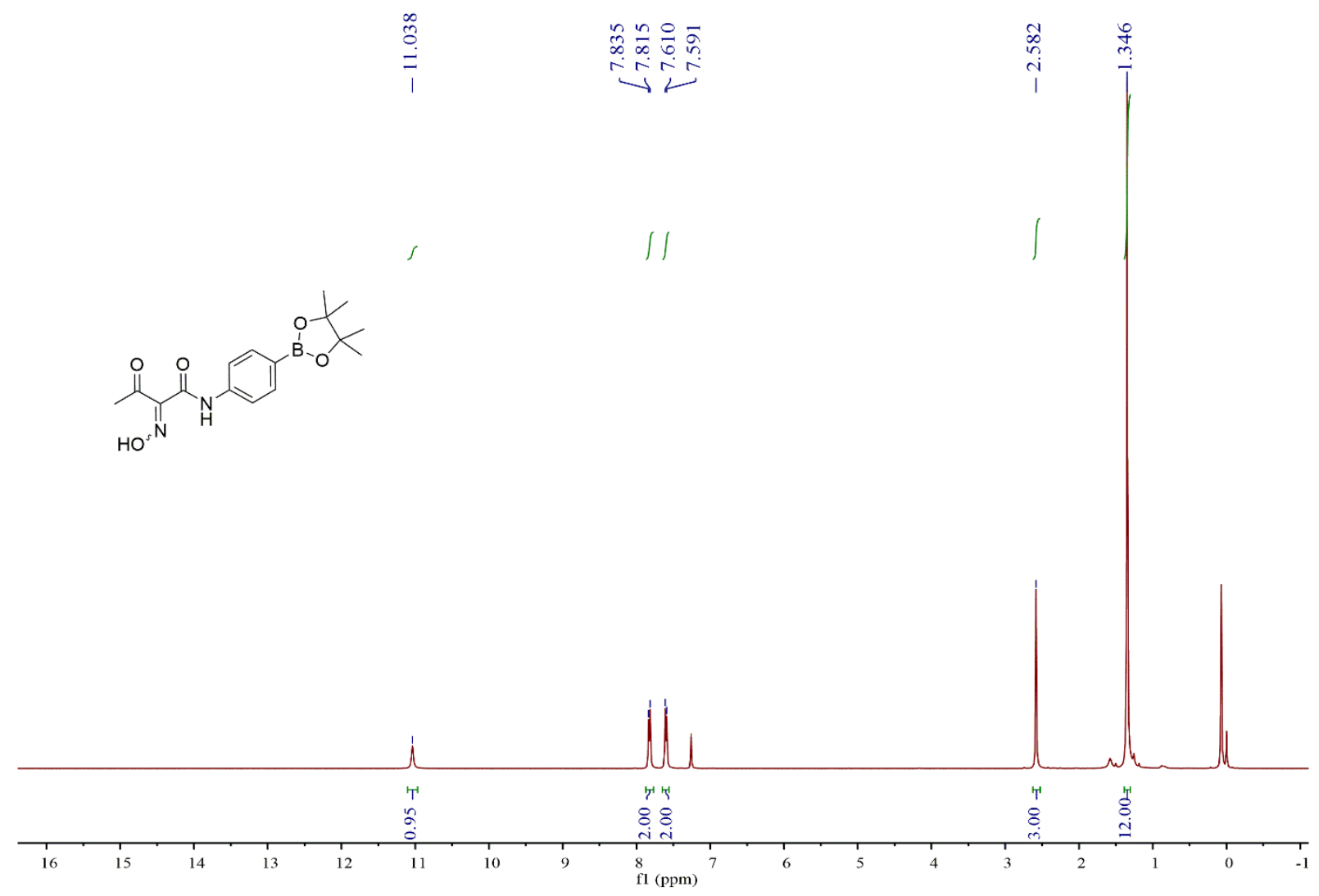

${ }^{1} \mathrm{H}$ NMR Spectrum of Compound $\mathbf{2 q}\left(400 \mathrm{MHz}, \mathrm{CDCl}_{3}\right)$

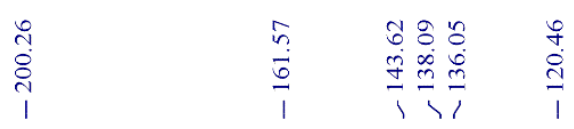

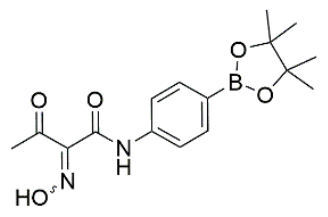

21

$\begin{array}{llllllllllll}210 & 200 & 190 & 180 & 170 & 160 & 150 & 140 & 130 & 120 & 110 & \begin{array}{c}100 \\ \mathrm{f} 1(\mathrm{ppm})\end{array}\end{array}$

${ }^{13} \mathrm{C}$ NMR Spectrum of Compound $\mathbf{2 q}\left(100 \mathrm{MHz}, \mathrm{CDCl}_{3}\right)$ 


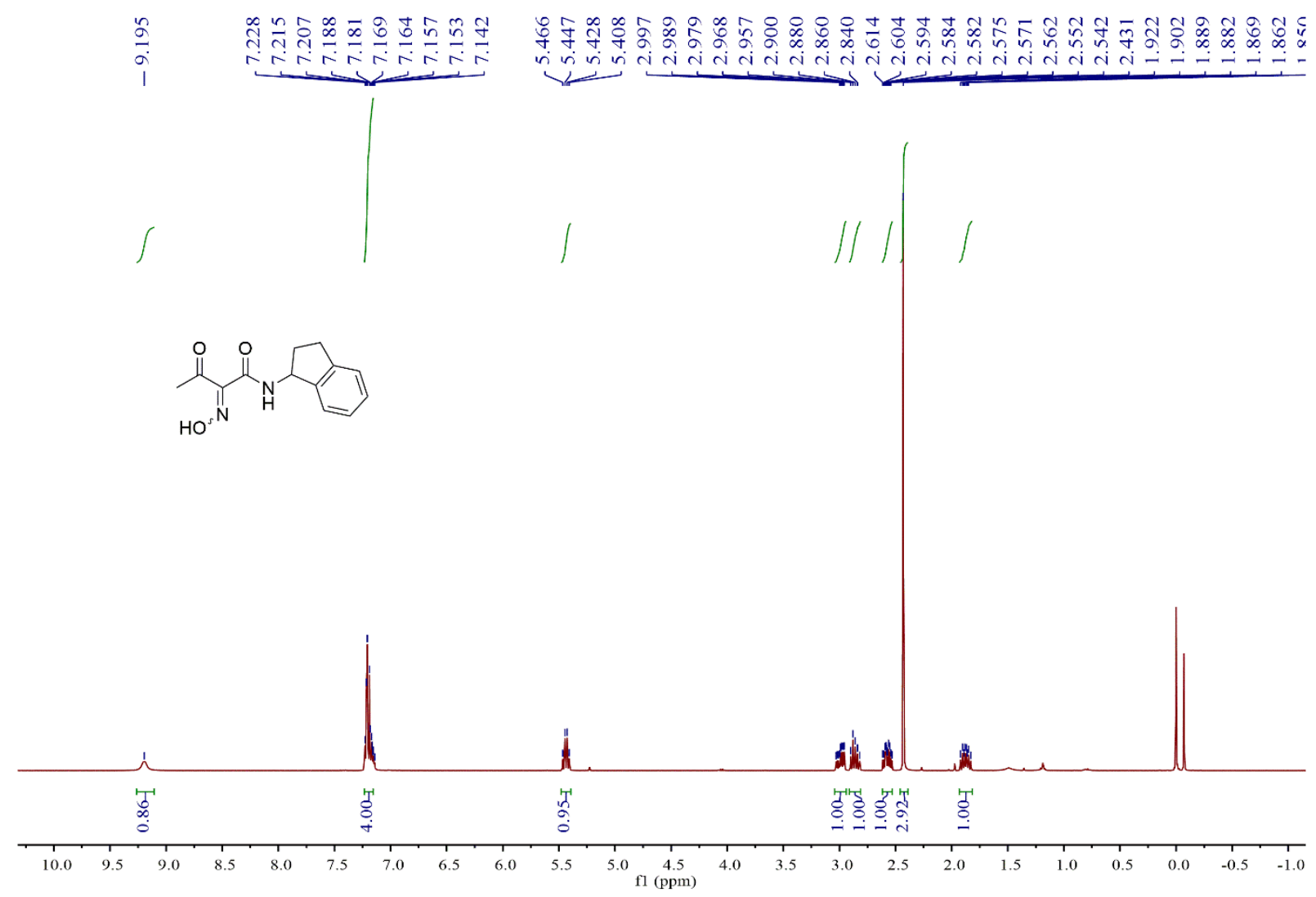

${ }^{1} \mathrm{H}$ NMR Spectrum of Compound $\mathbf{2} \mathbf{u}\left(400 \mathrm{MHz} \mathrm{CDCl}_{3}\right)$

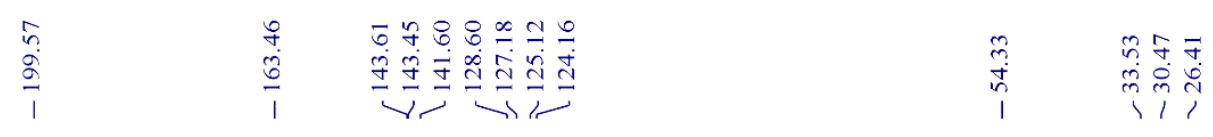
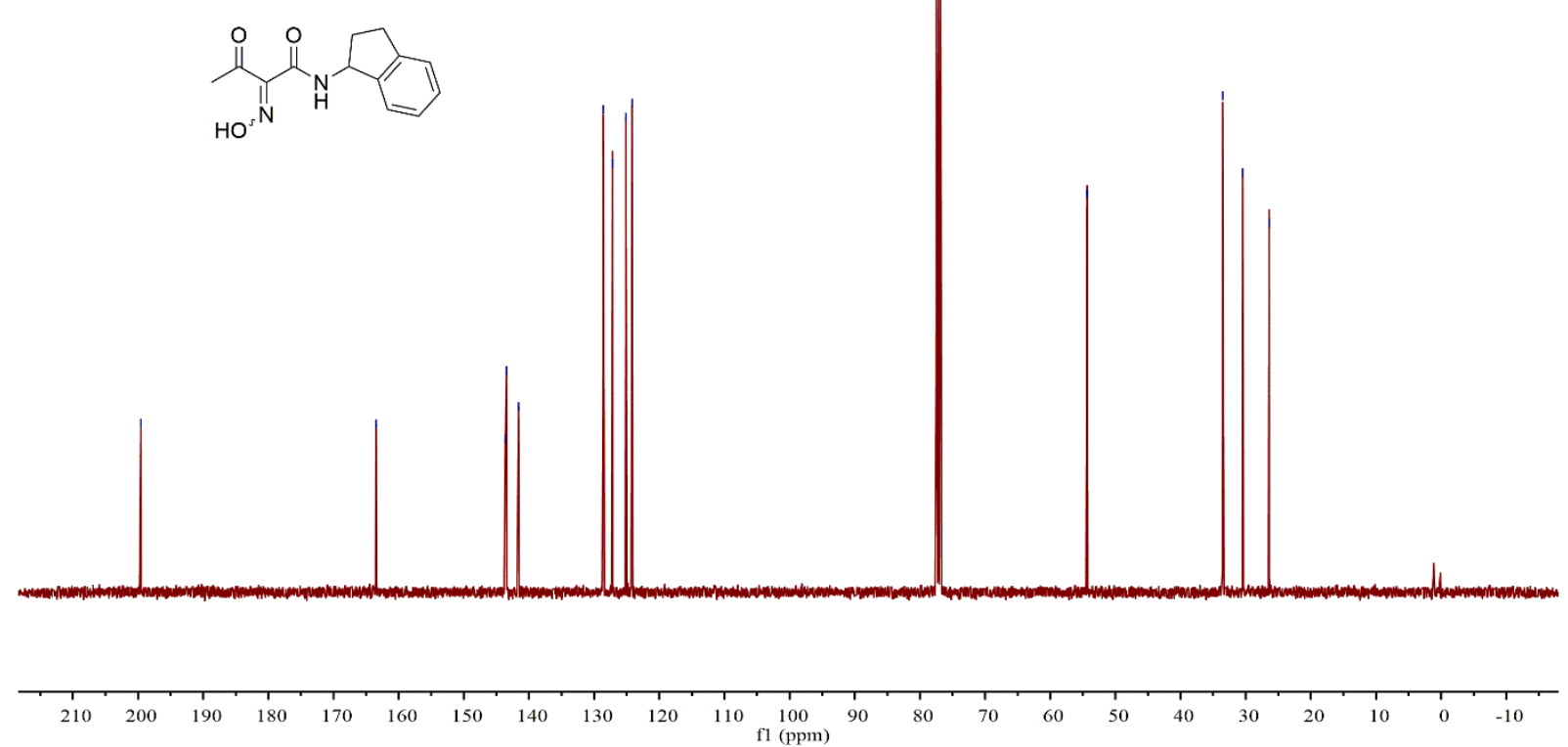

${ }^{13} \mathrm{C}$ NMR Spectrum of Compound $\mathbf{2 u}\left(100 \mathrm{MHz}, \mathrm{CDCl}_{3}\right)$ 


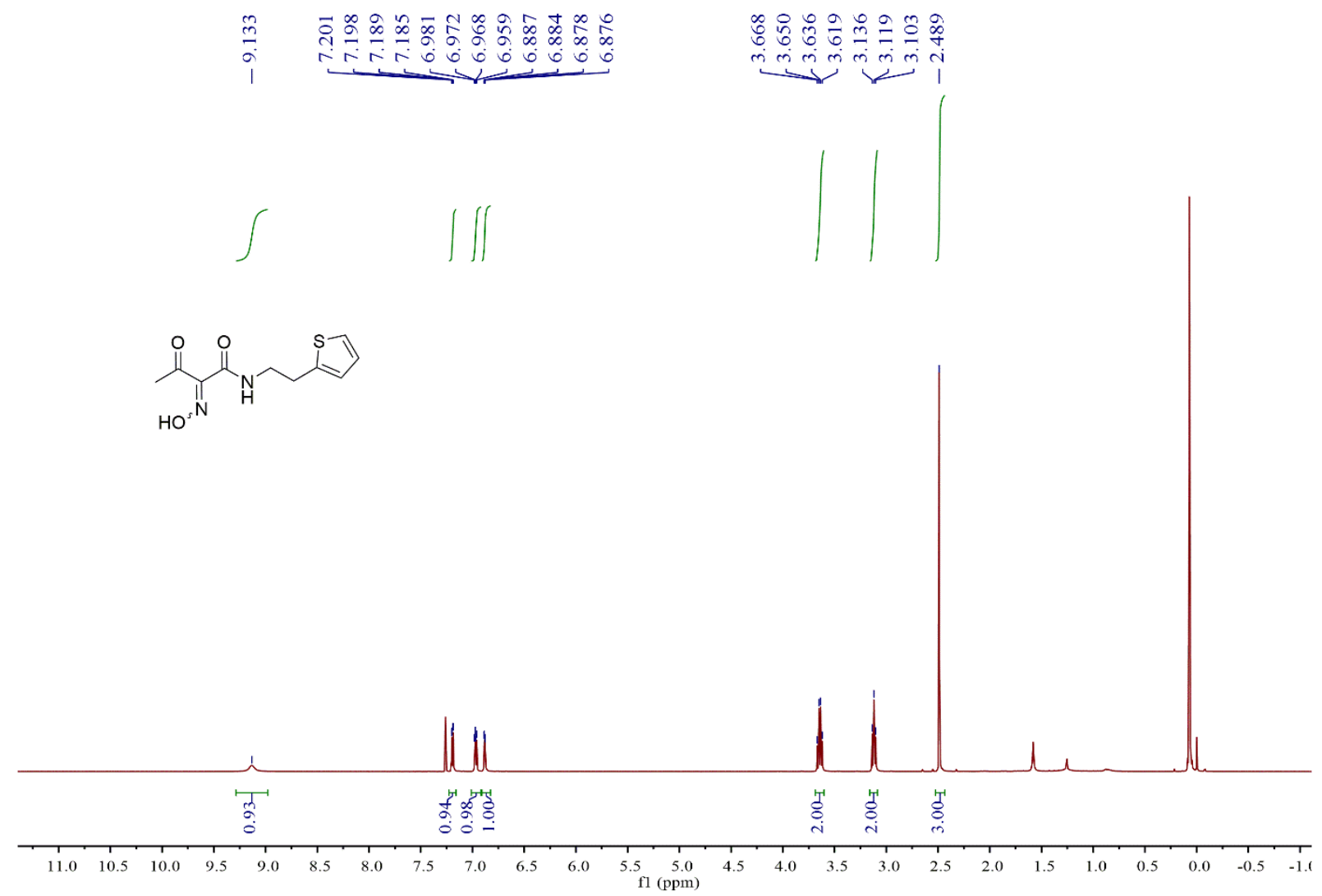

${ }^{1} \mathrm{H}$ NMR Spectrum of Compound $\mathbf{2 z}\left(400 \mathrm{MHz}, \mathrm{CDCl}_{3}\right)$

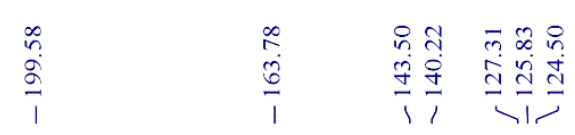

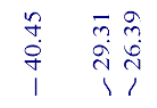
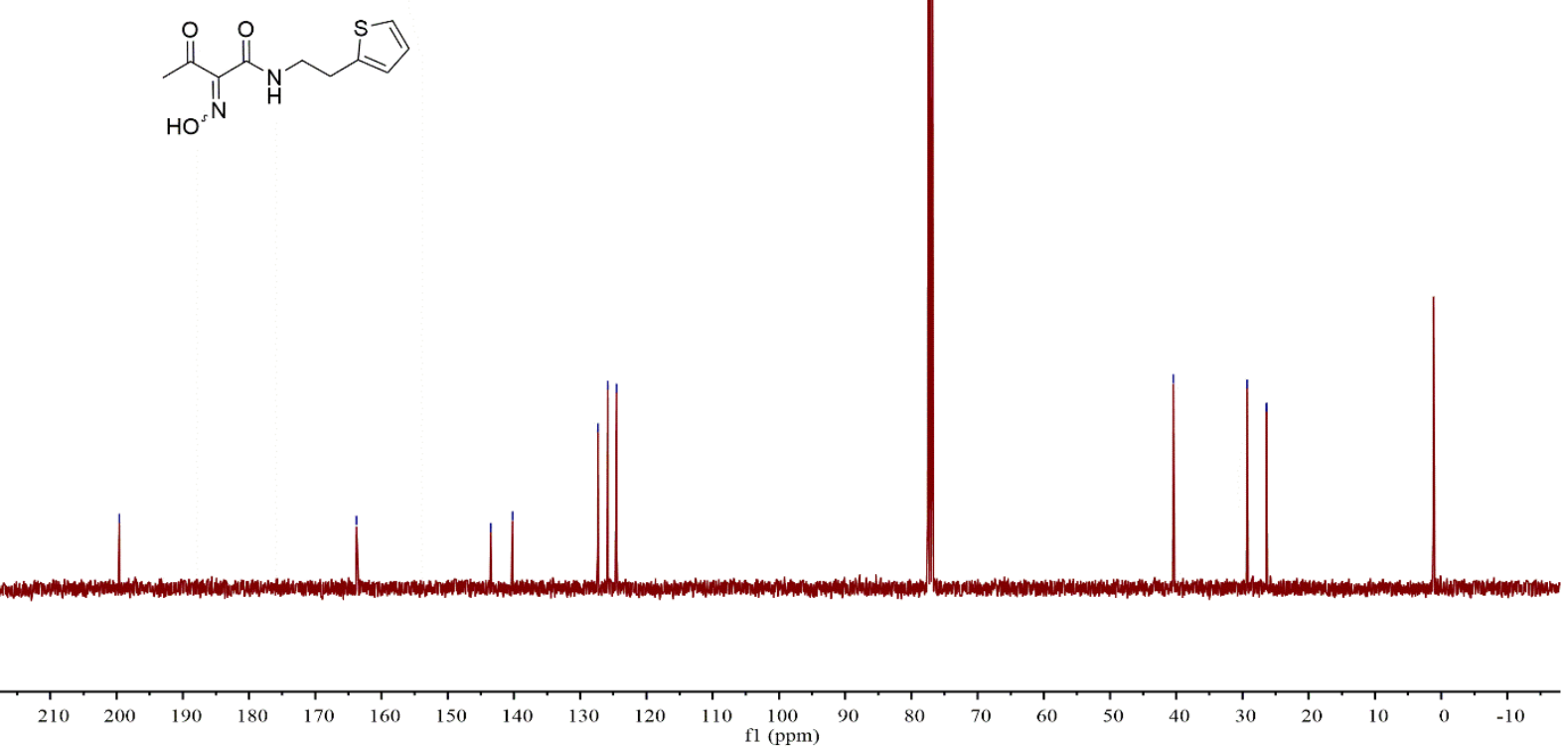

${ }^{13} \mathrm{C}$ NMR Spectrum of Compound $2 \mathbf{z}\left(100 \mathrm{MHz}, \mathrm{CDCl}_{3}\right)$ 


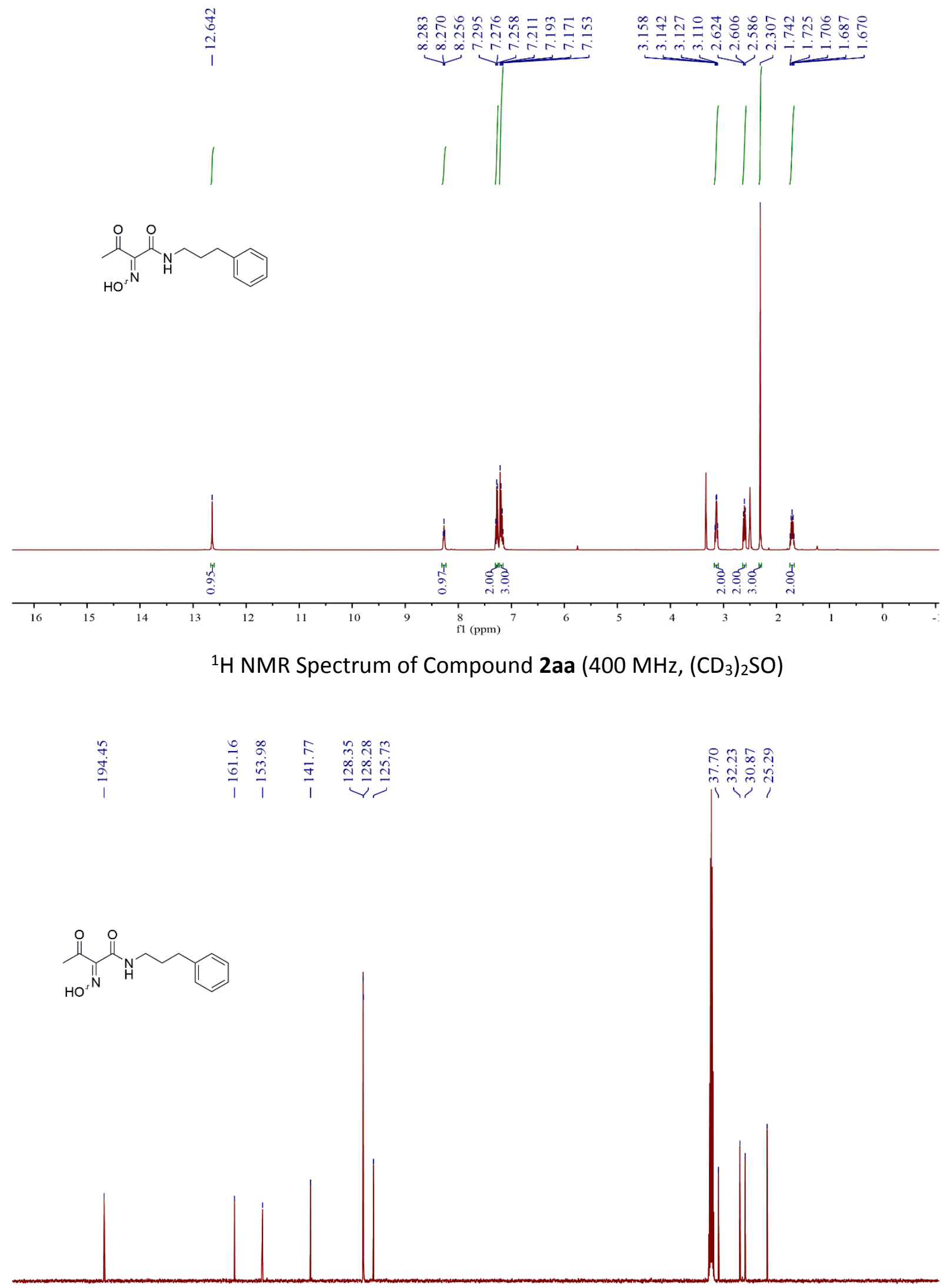

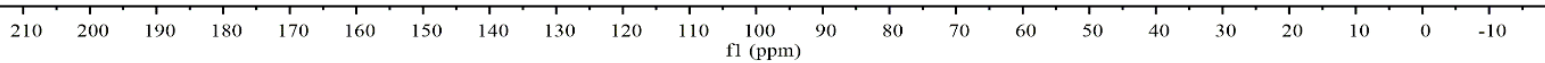

${ }^{13} \mathrm{C}$ NMR Spectrum of Compound 2aa $\left(100 \mathrm{MHz},\left(\mathrm{CD}_{3}\right)_{2} \mathrm{SO}\right)$ 


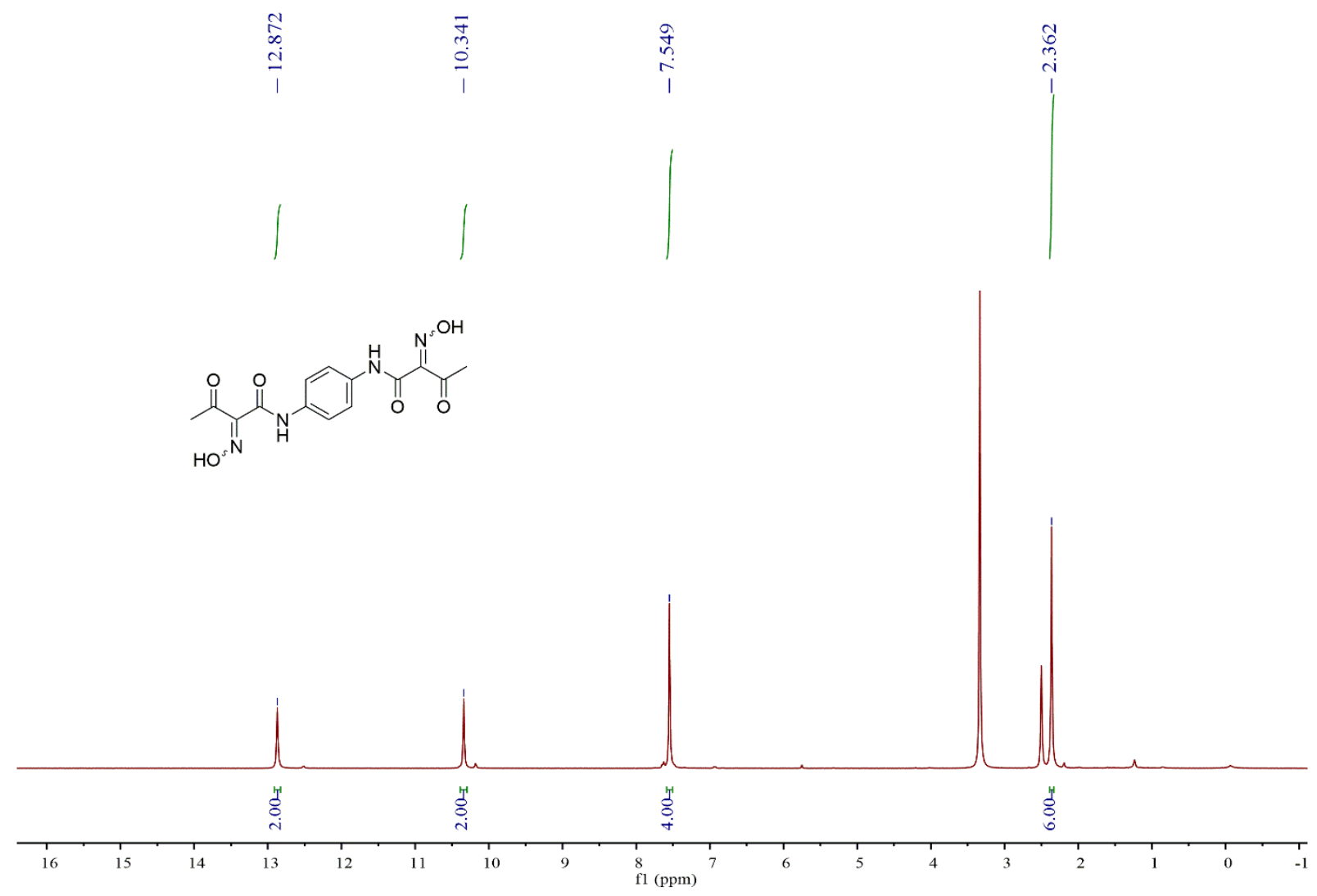

${ }^{1} \mathrm{H}$ NMR Spectrum of Compound $2 \mathrm{ab}\left(400 \mathrm{MHz},\left(\mathrm{CD}_{3}\right)_{2} \mathrm{SO}\right)$

$\begin{array}{lllll}1 & \frac{1}{2} & 1 & 1 & 1\end{array}$
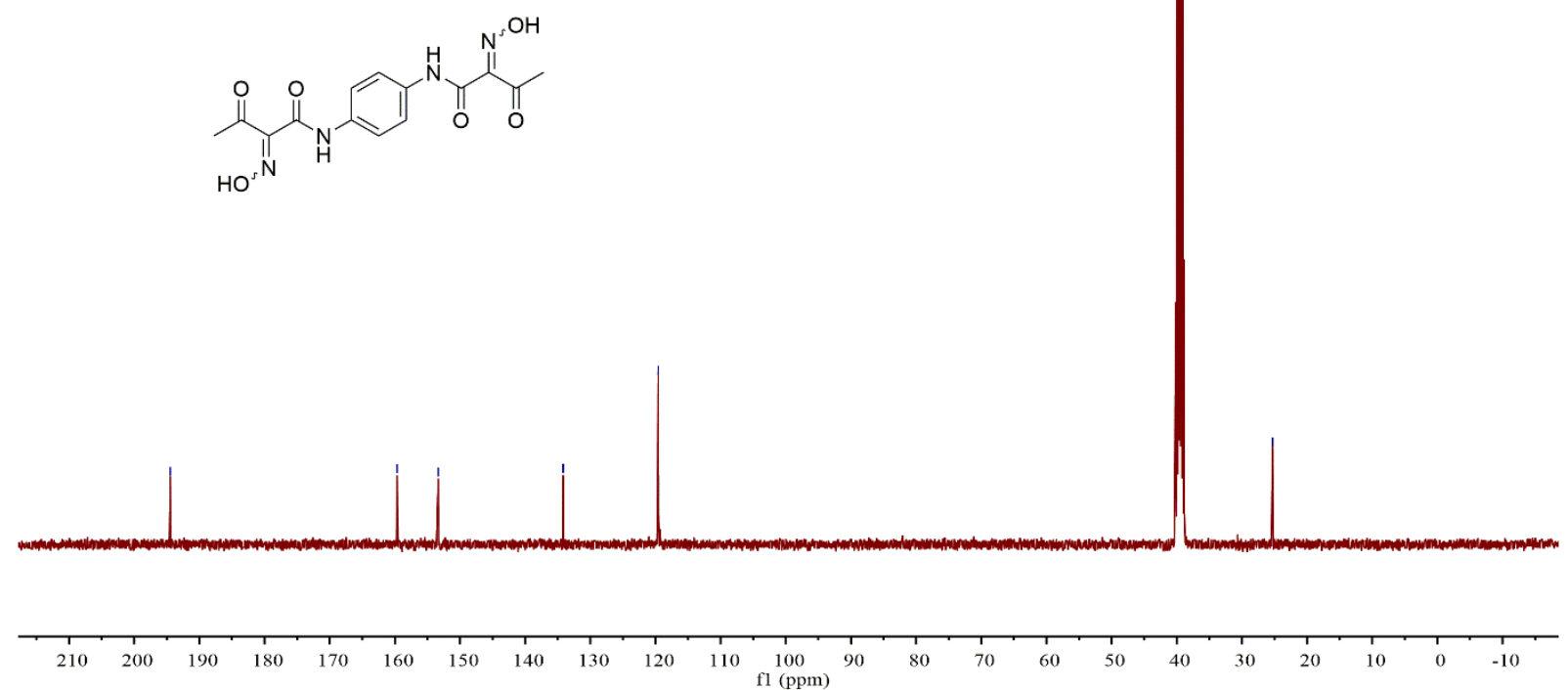

${ }^{13} \mathrm{C}$ NMR Spectrum of Compound $2 \mathrm{ab}\left(100 \mathrm{MHz},\left(\mathrm{CD}_{3}\right)_{2} \mathrm{SO}\right)$ 


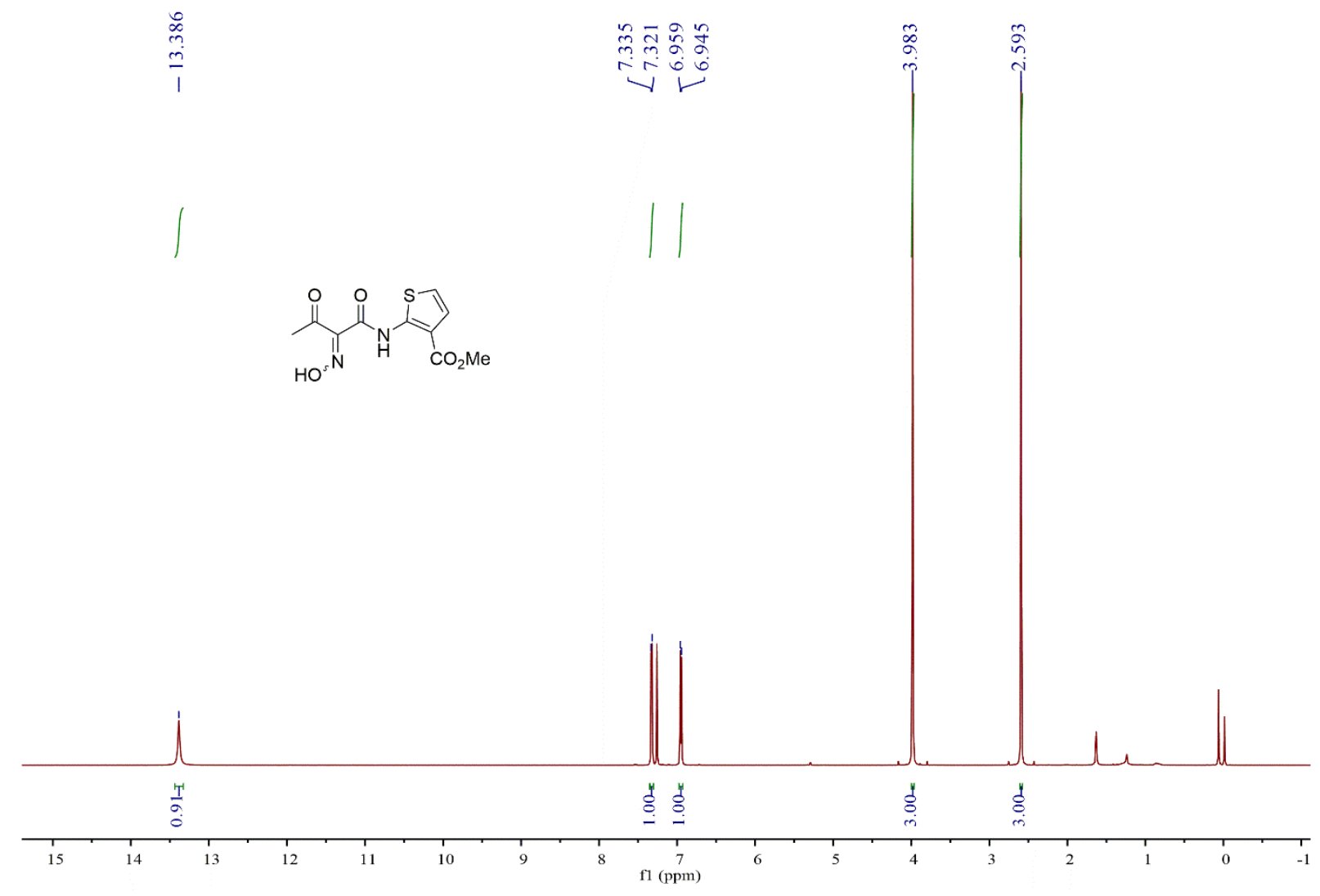

${ }^{1} \mathrm{H}$ NMR Spectrum of Compound 2 af $\left(400 \mathrm{MHz} \mathrm{CDCl}_{3}\right)$

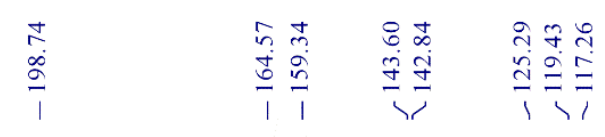
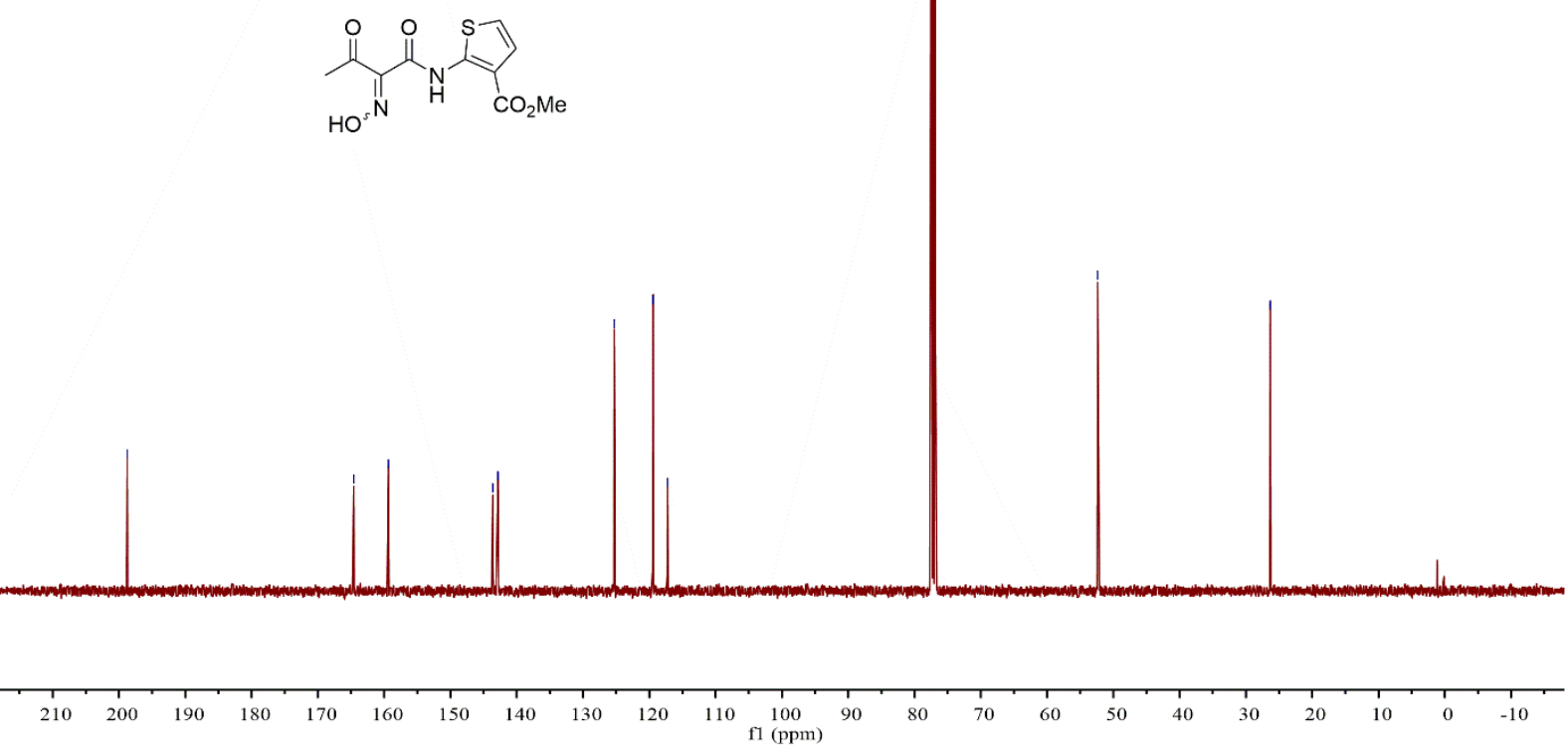

${ }^{13} \mathrm{C}$ NMR Spectrum of Compound 2af $\left(100 \mathrm{MHz}, \mathrm{CDCl}_{3}\right)$ 


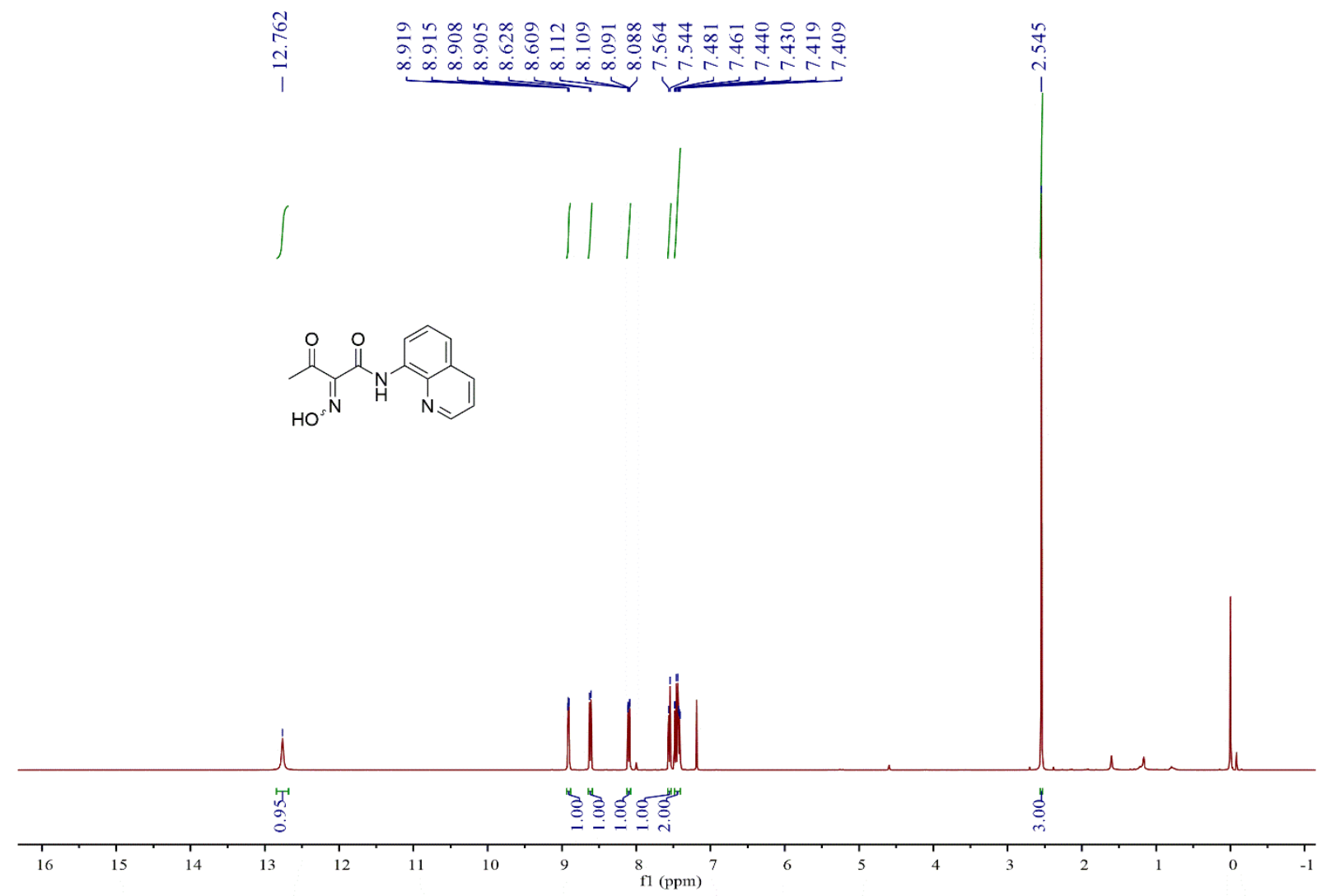

${ }^{1} \mathrm{H}$ NMR Spectrum of Compound $2 \mathrm{ag}\left(400 \mathrm{MHz}, \mathrm{CDCl}_{3}\right)$
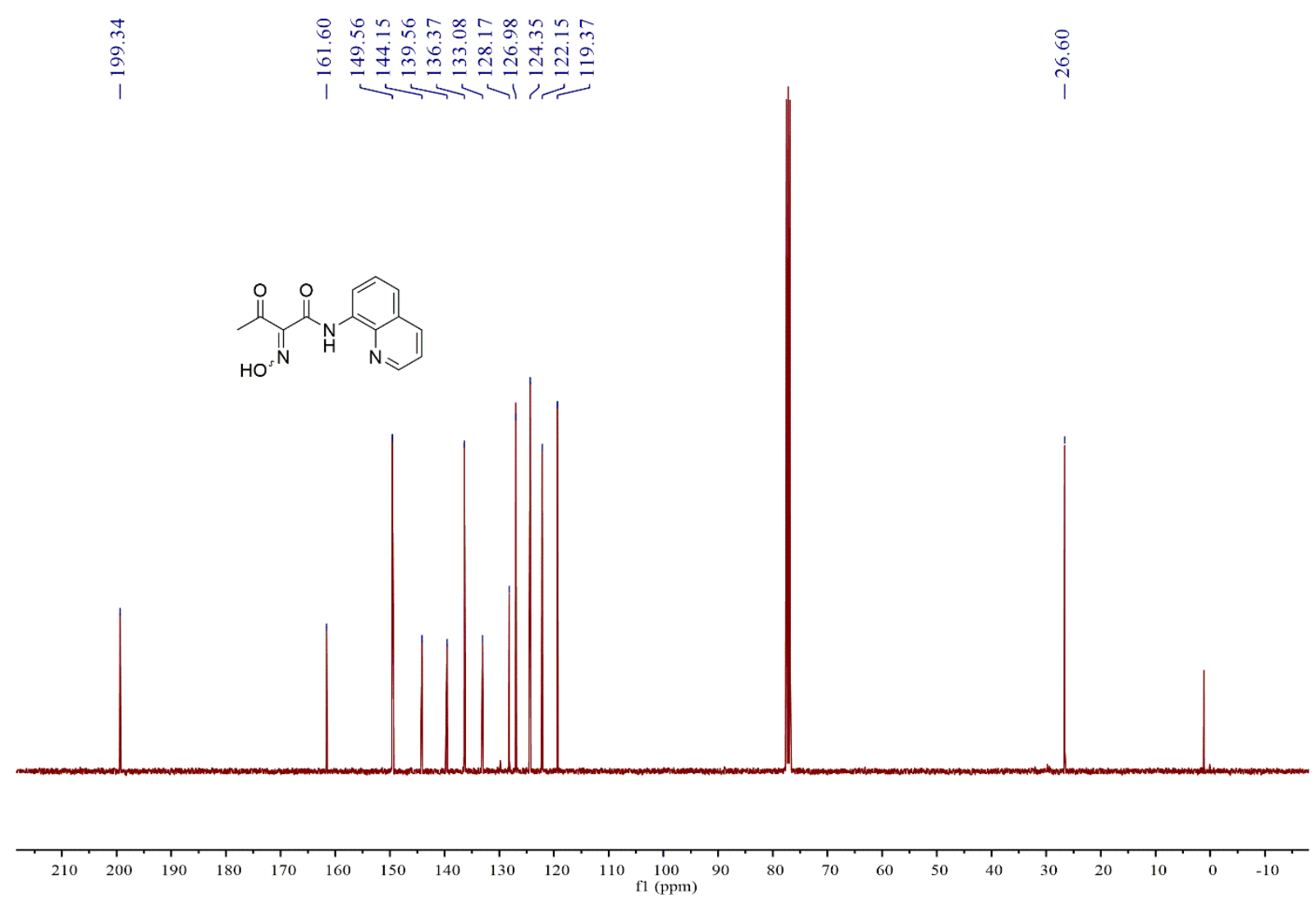

${ }^{13} \mathrm{C}$ NMR Spectrum of Compound $2 \mathrm{ag}\left(100 \mathrm{MHz}, \mathrm{CDCl}_{3}\right)$ 


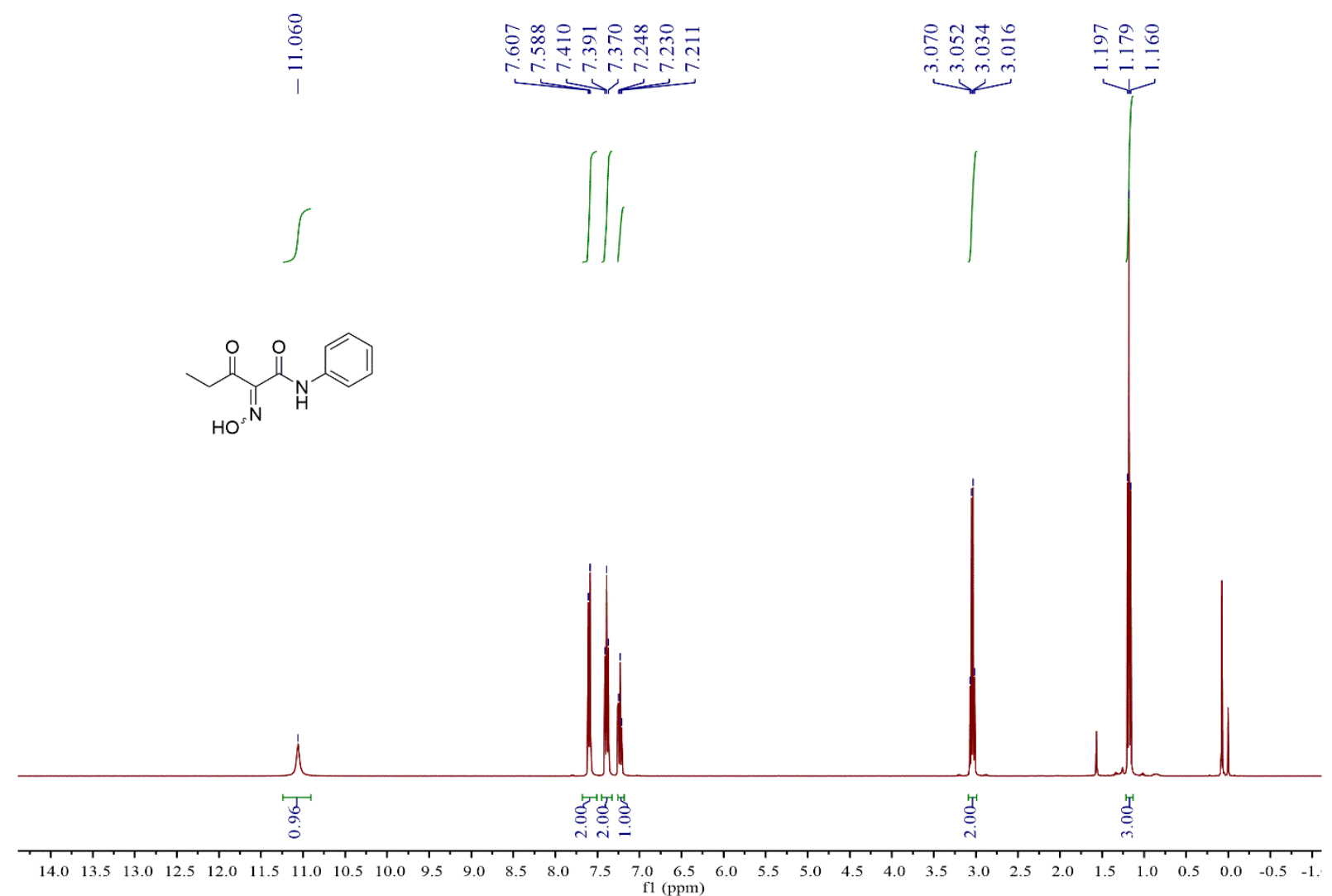

${ }^{1} \mathrm{H}$ NMR Spectrum of Compound $2 \mathrm{ah}\left(400 \mathrm{MHz}, \mathrm{CDCl}_{3}\right)$

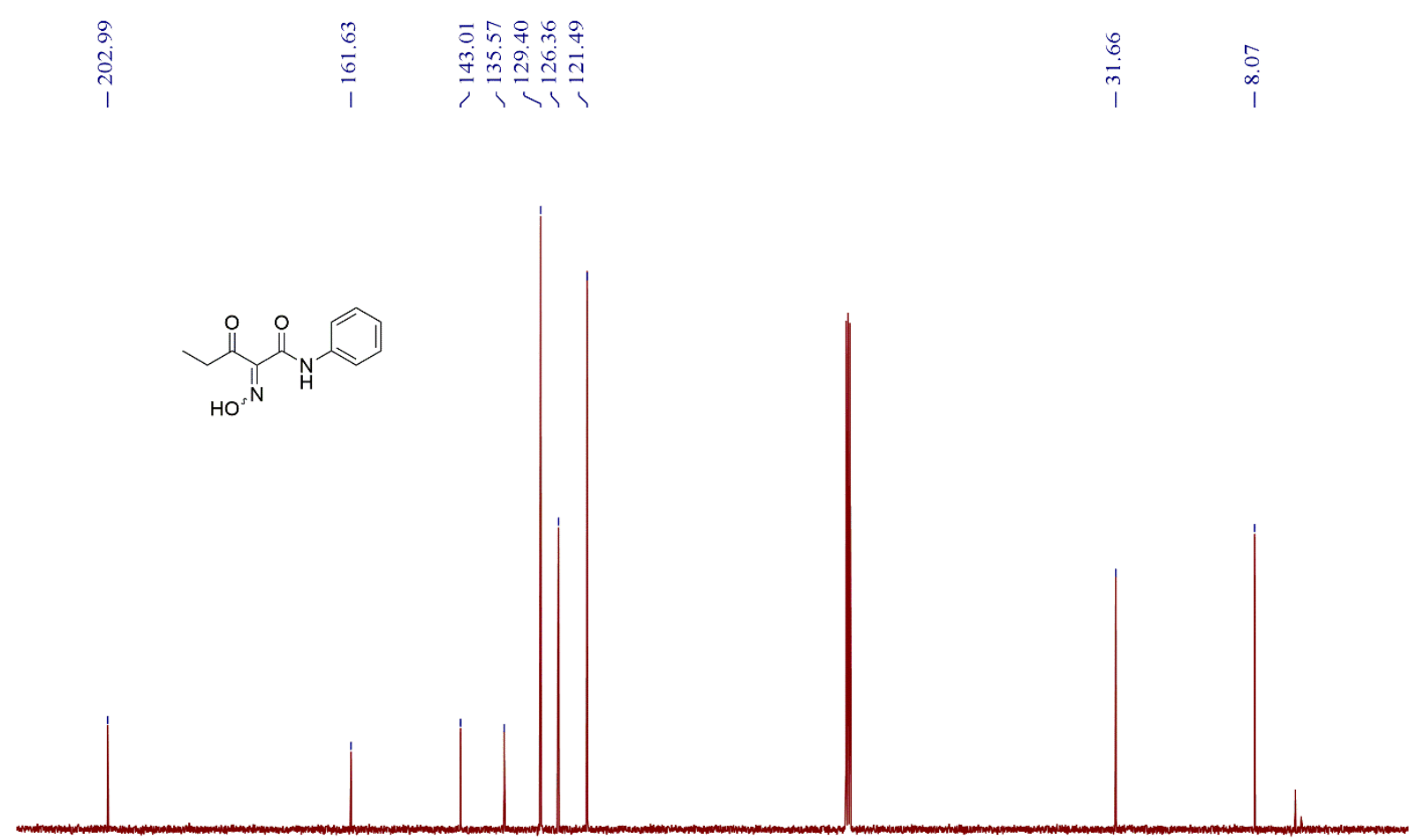

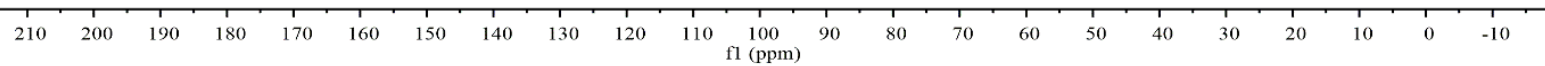

${ }^{13} \mathrm{C}$ NMR Spectrum of Compound $2 \mathrm{ah}\left(100 \mathrm{MHz}^{\mathrm{CDCl}}\right)_{3}$ 

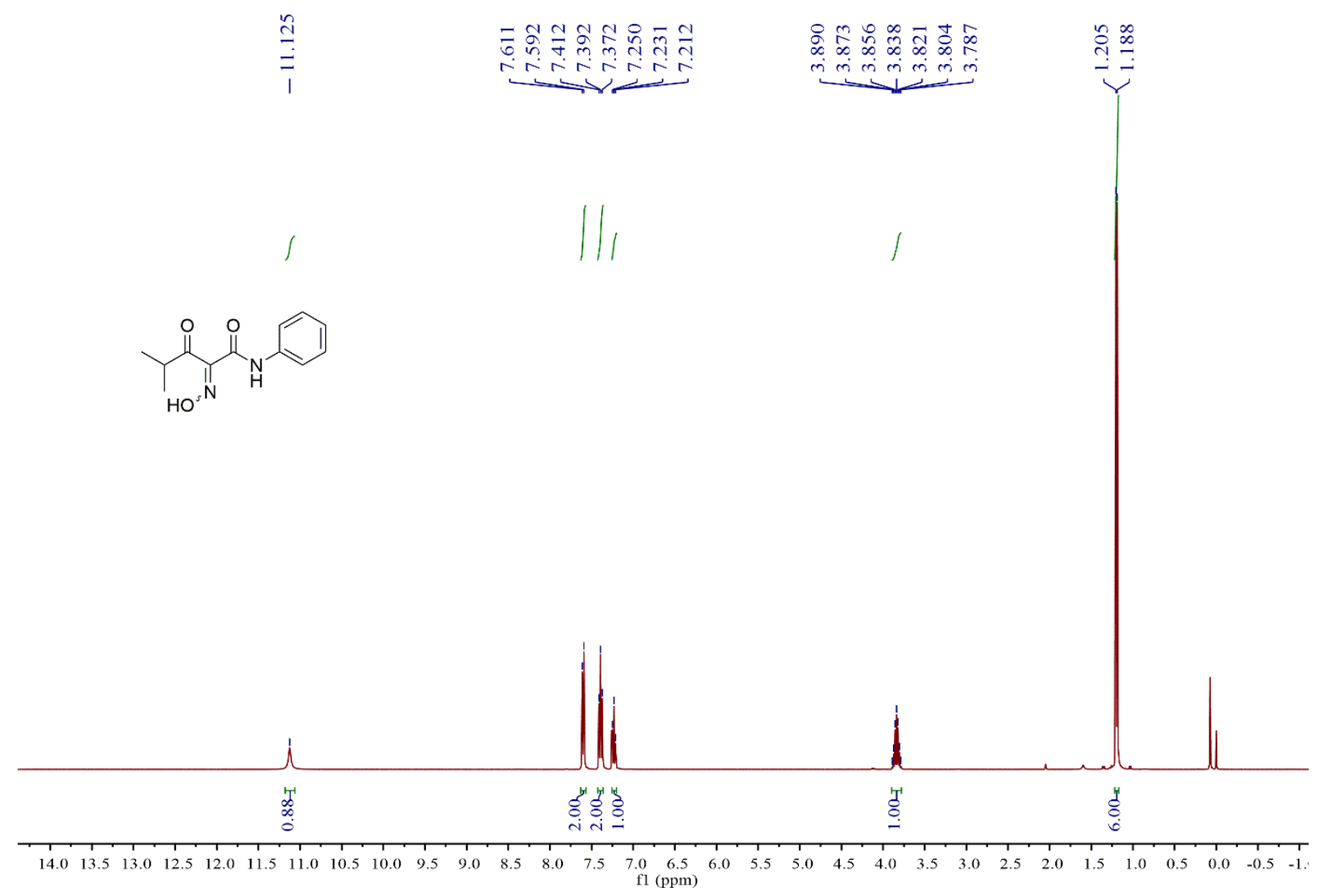

${ }^{1} \mathrm{H}$ NMR Spectrum of Compound 2ai (400 MHz, $\mathrm{CDCl}_{3}$ )
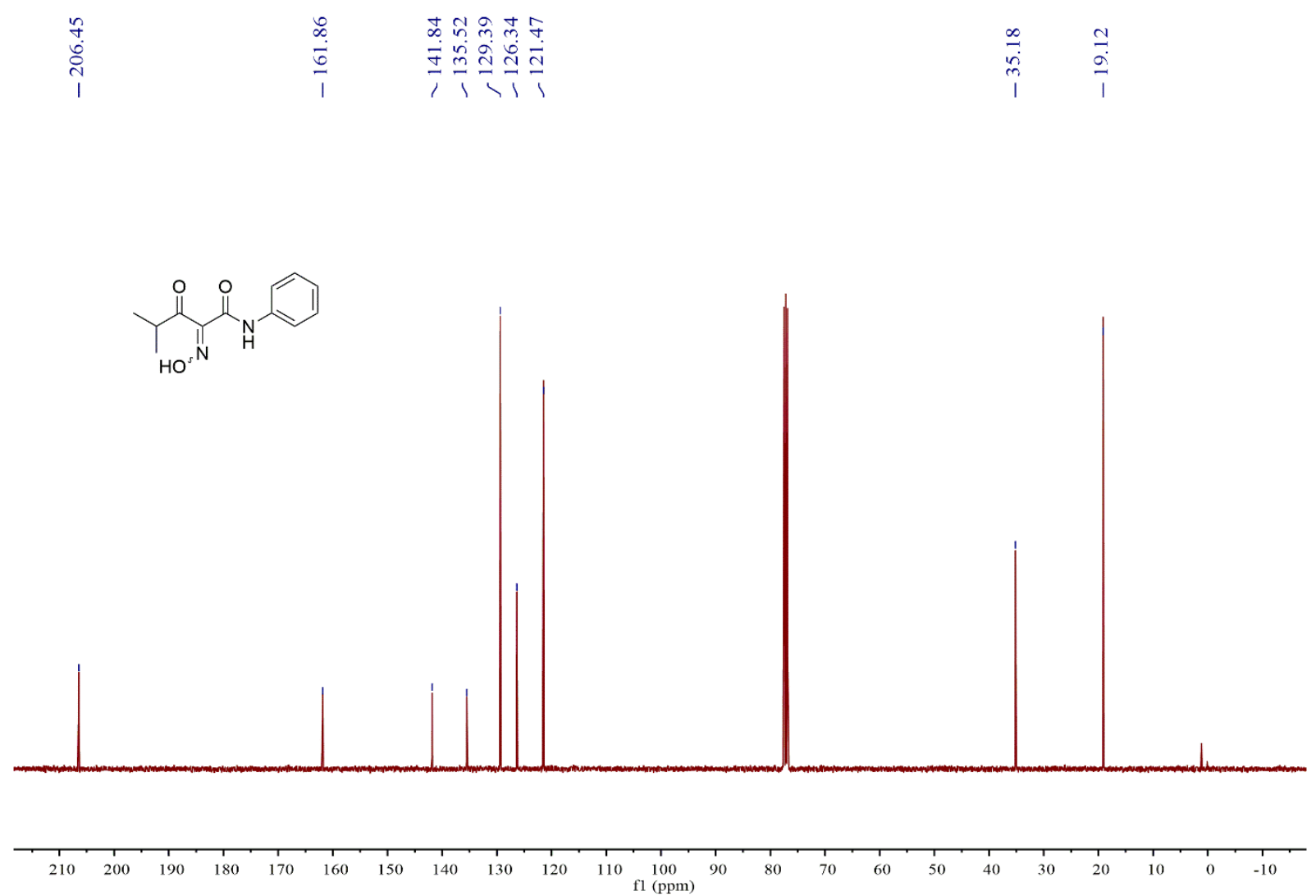

${ }^{13} \mathrm{C}$ NMR Spectrum of Compound 2ai (100 MHz, $\mathrm{CDCl}_{3}$ ) 

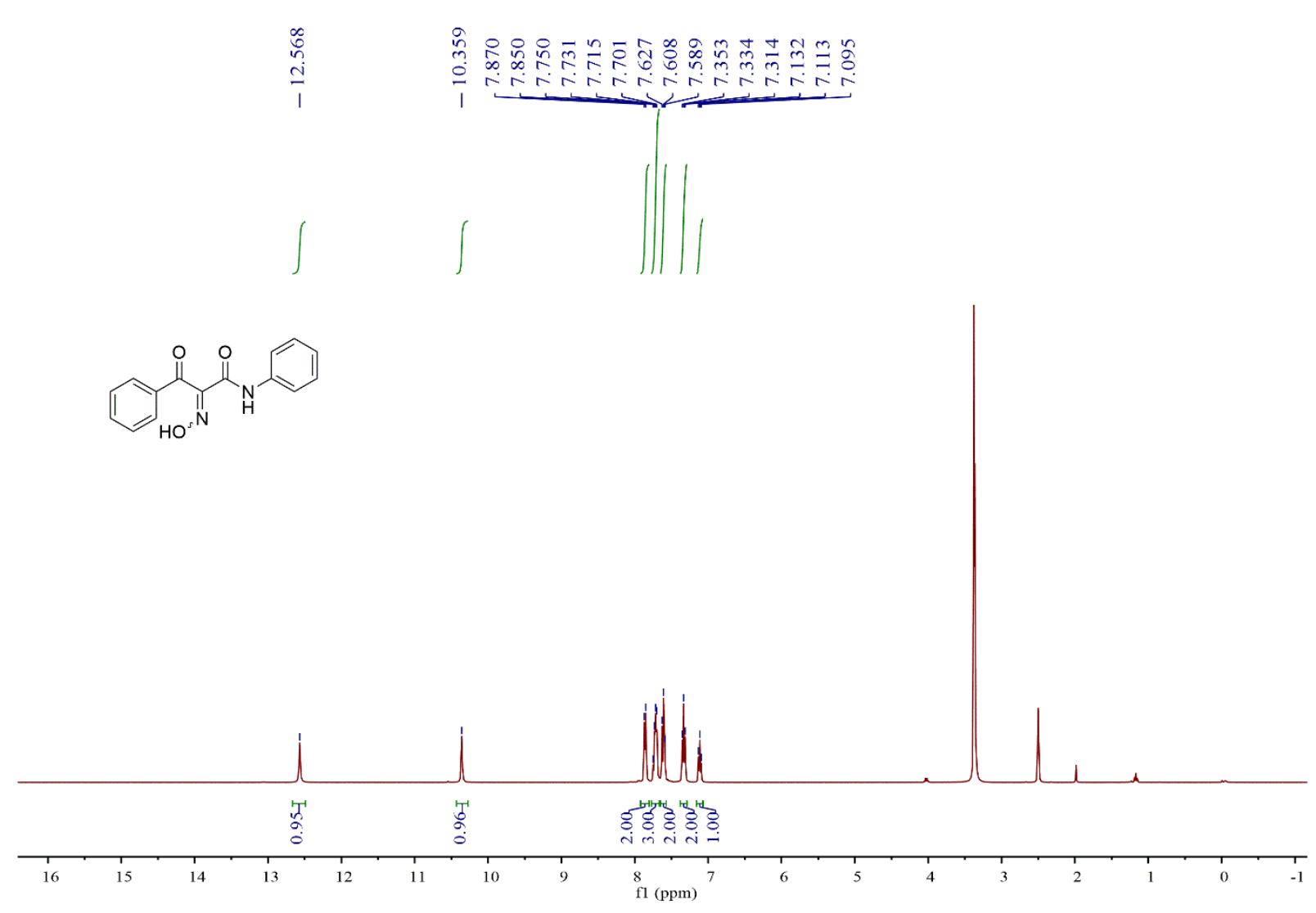

${ }^{1} \mathrm{H}$ NMR Spectrum of Compound 2aj (400 MHz, $\left.\left(\mathrm{CD}_{3}\right)_{2} \mathrm{SO}\right)$
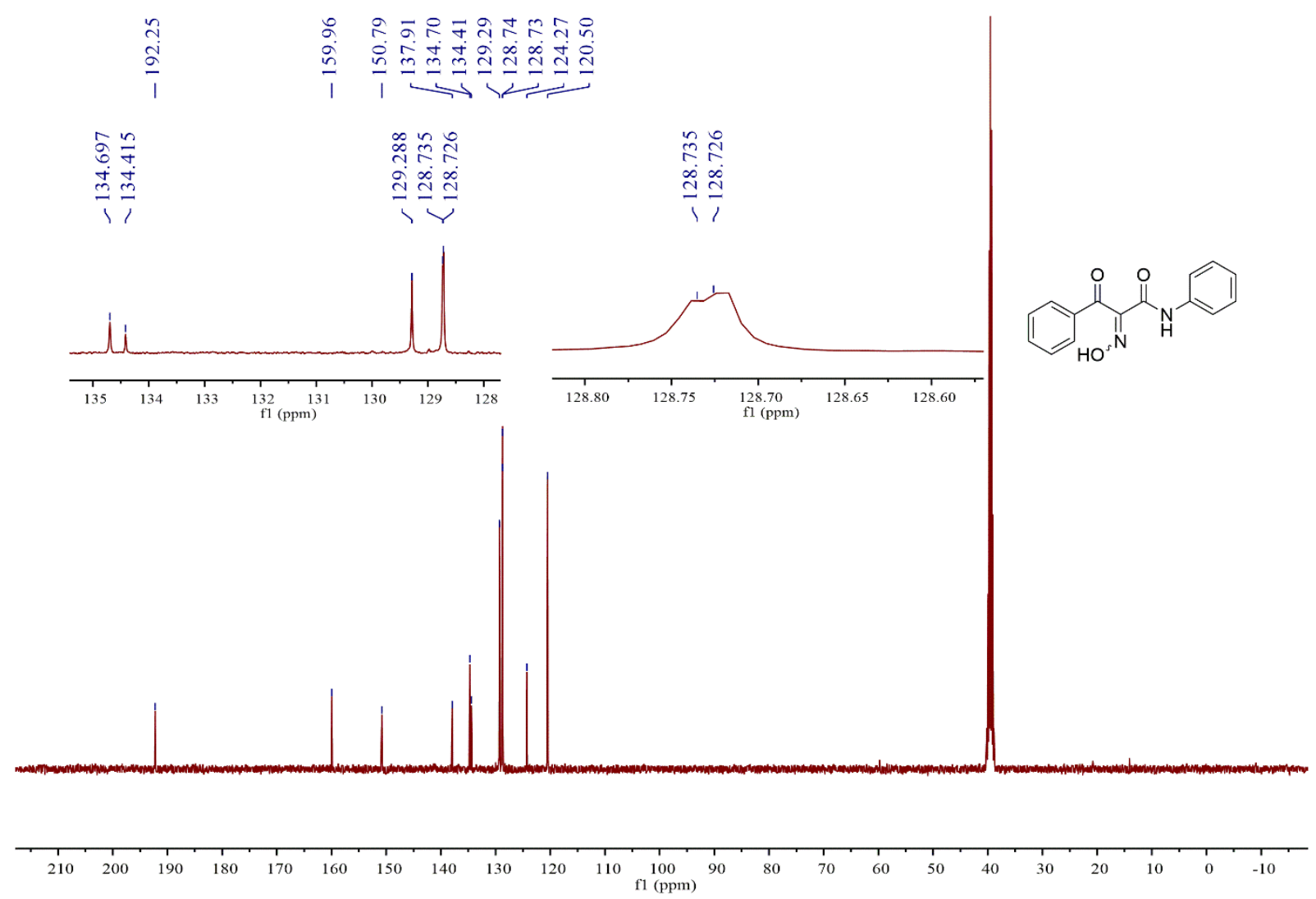

${ }^{13} \mathrm{C}$ NMR Spectrum of Compound 2aj (100 MHz, $\left.\left(\mathrm{CD}_{3}\right)_{2} \mathrm{SO}\right)$ 


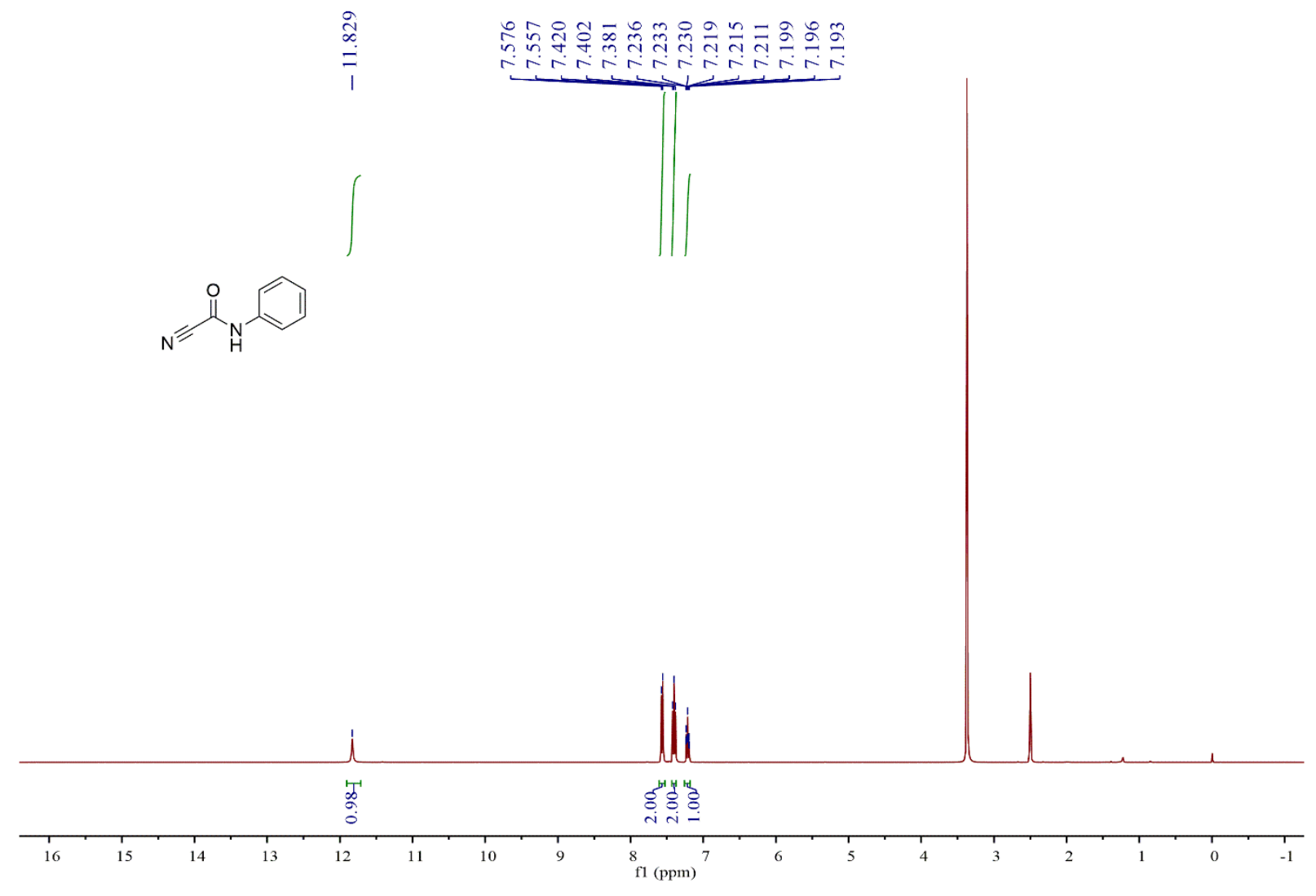

${ }^{1} \mathrm{H}$ NMR Spectrum of Compound $3 \mathrm{a}\left(400 \mathrm{MHz},\left(\mathrm{CD}_{3}\right)_{2} \mathrm{SO}\right)$
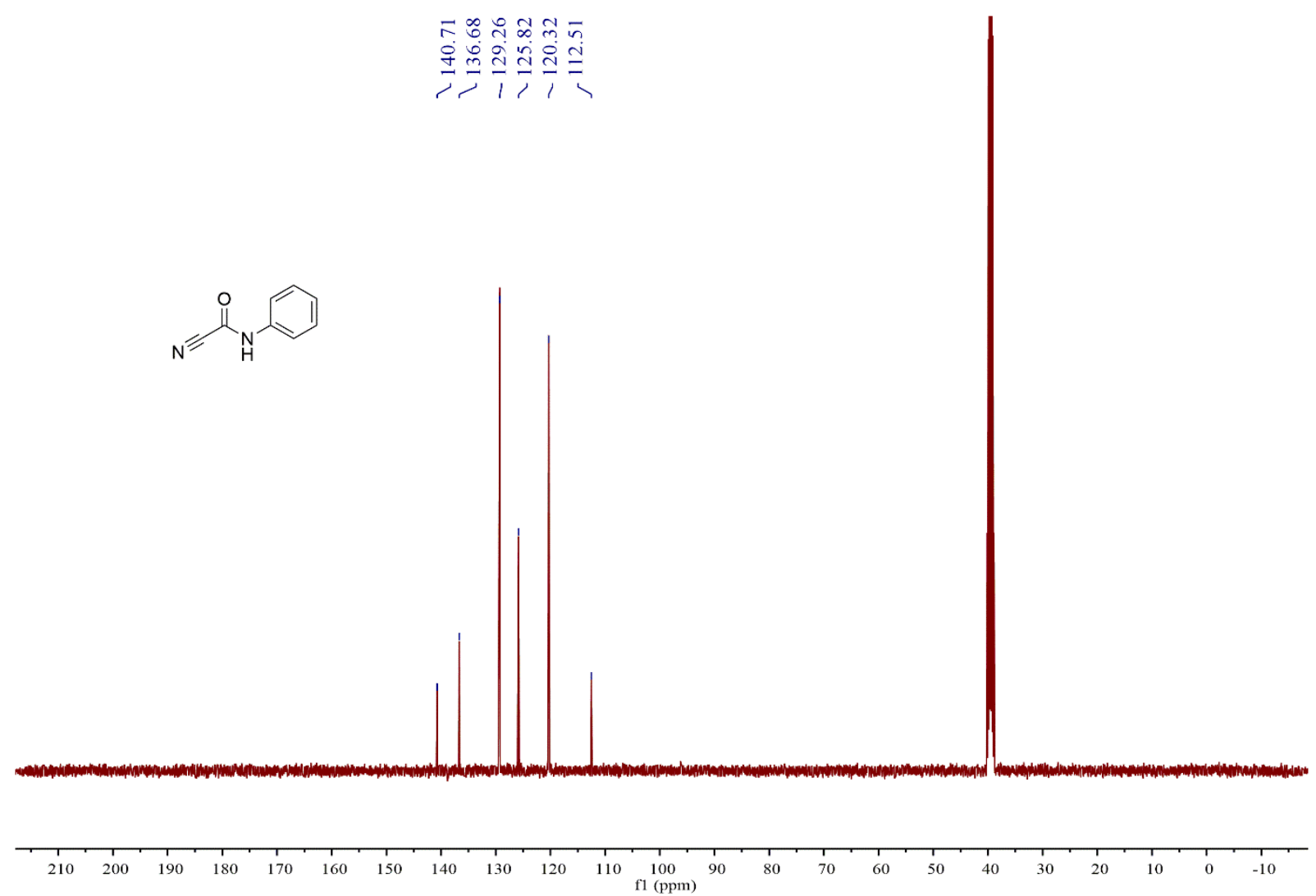

${ }^{13} \mathrm{C}$ NMR Spectrum of Compound $3 \mathrm{a}\left(100 \mathrm{MHz},\left(\mathrm{CD}_{3}\right)_{2} \mathrm{SO}\right)$ 

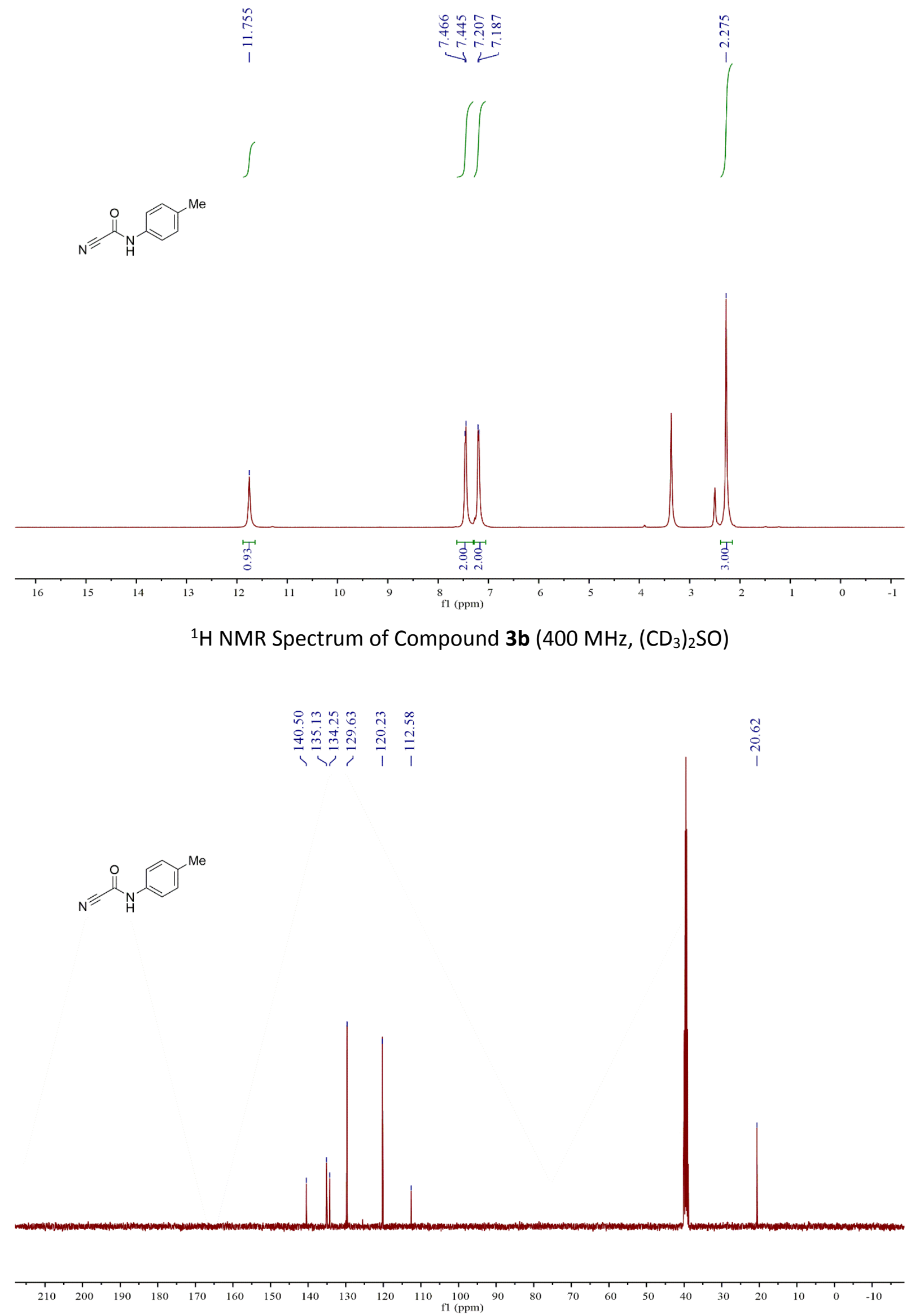

${ }^{13} \mathrm{C}$ NMR Spectrum of Compound $3 \mathbf{b}\left(100 \mathrm{MHz},\left(\mathrm{CD}_{3}\right)_{2} \mathrm{SO}\right)$ 


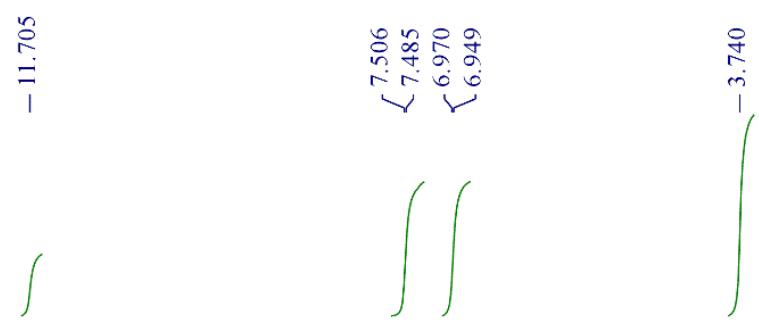

OMe

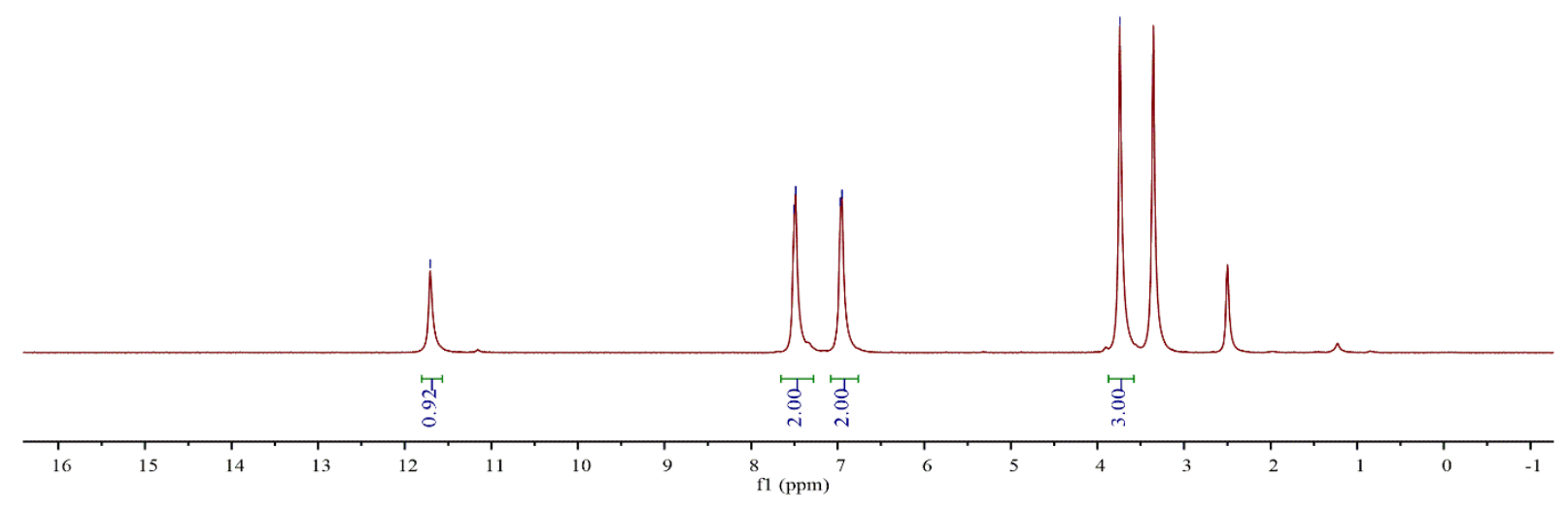

${ }^{1} \mathrm{H}$ NMR Spectrum of Compound $3 \mathrm{c}\left(400 \mathrm{MHz},\left(\mathrm{CD}_{3}\right)_{2} \mathrm{SO}\right)$
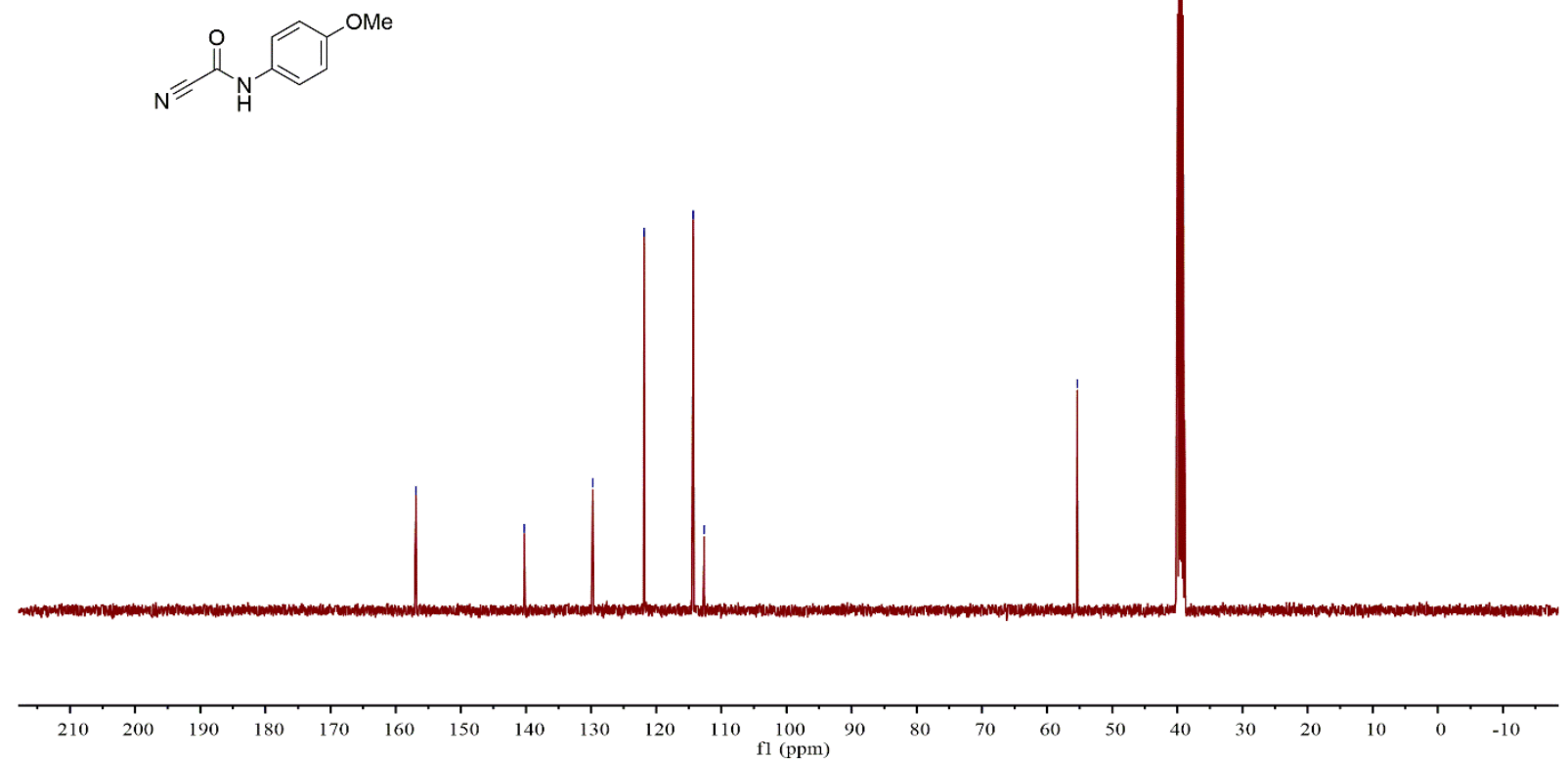

${ }^{13} \mathrm{C}$ NMR Spectrum of Compound $3 \mathrm{c}\left(100 \mathrm{MHz},\left(\mathrm{CD}_{3}\right)_{2} \mathrm{SO}\right)$ 
考导毒学总

$45 \pi 55$

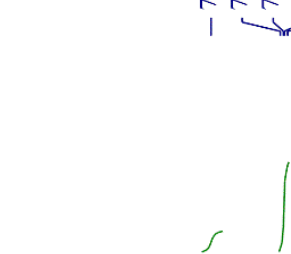

$N=\overbrace{}^{B}$
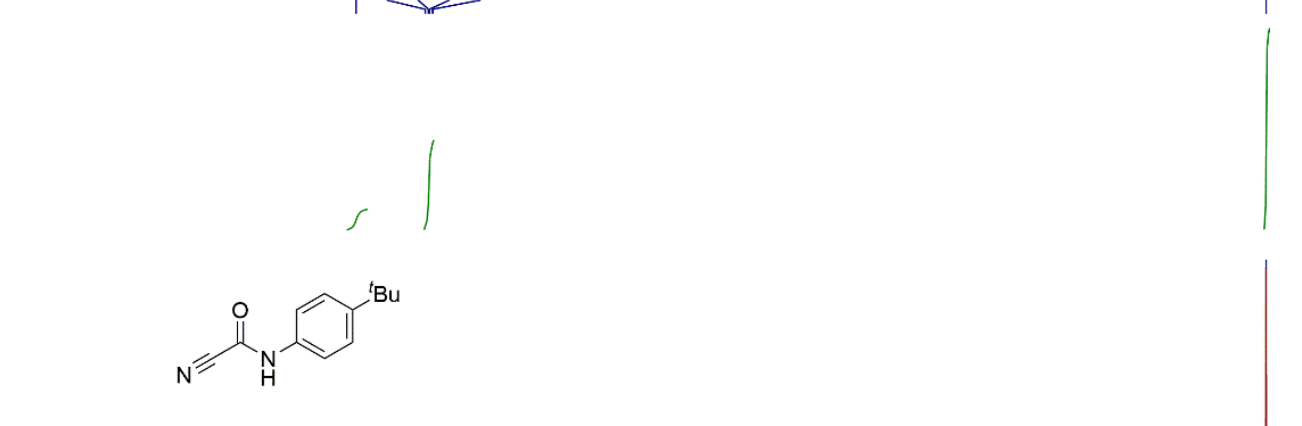

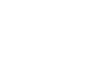

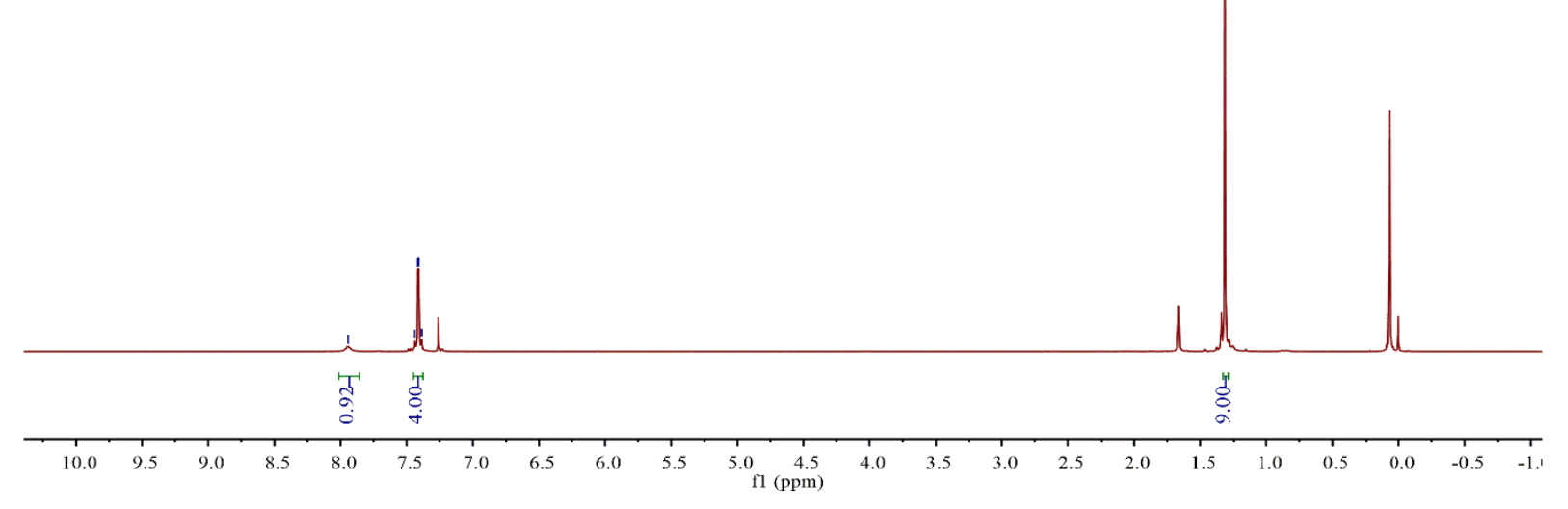

${ }^{1} \mathrm{H}$ NMR Spectrum of Compound $\mathbf{3 d}\left(400 \mathrm{MHz}, \mathrm{CDCl}_{3}\right)$
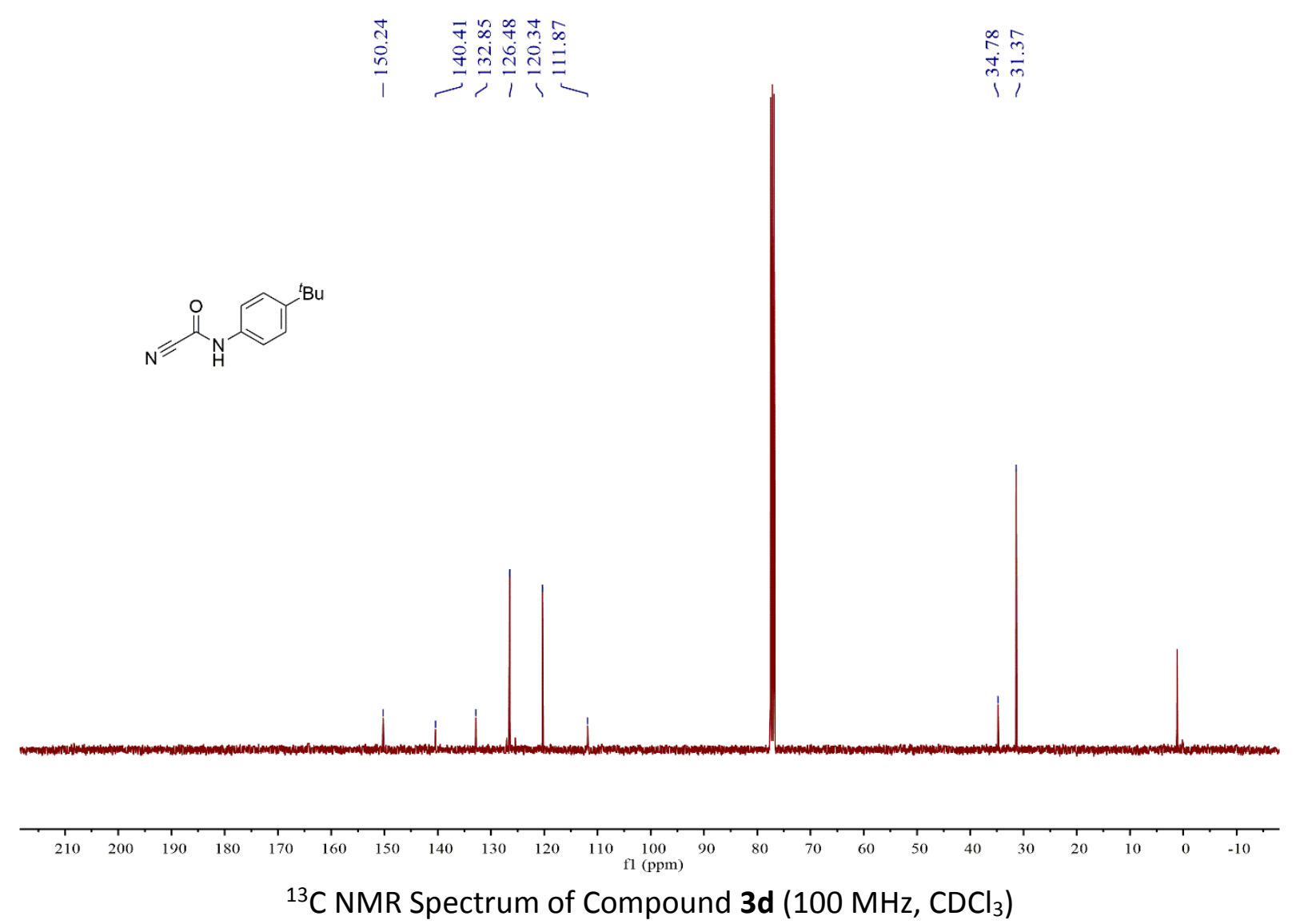


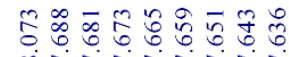

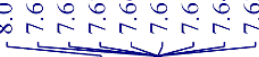<smiles>N#CC(=O)Nc1ccc(C(F)(F)F)cc1</smiles>

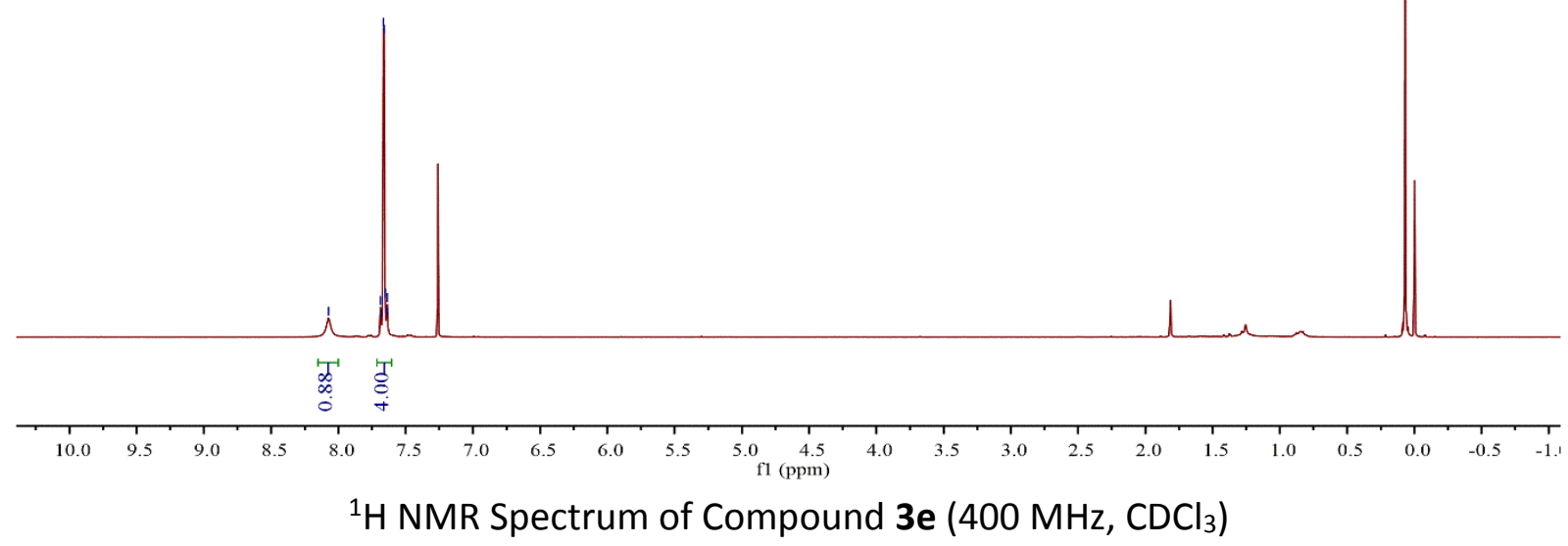

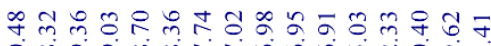

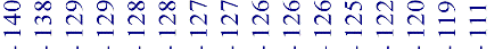
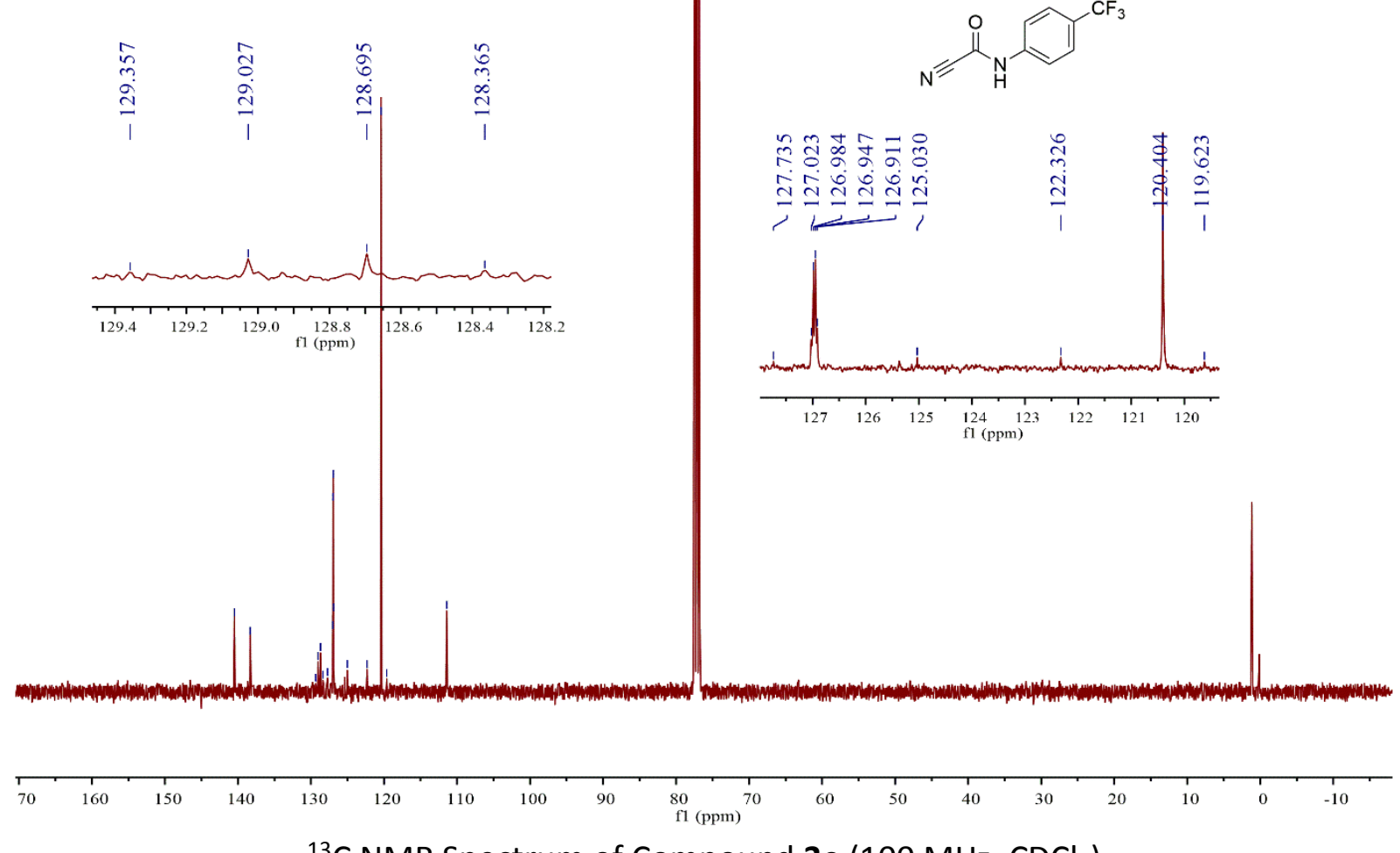

${ }^{13} \mathrm{C}$ NMR Spectrum of Compound $3 \mathrm{e}\left(100 \mathrm{MHz}, \mathrm{CDCl}_{3}\right)$ 


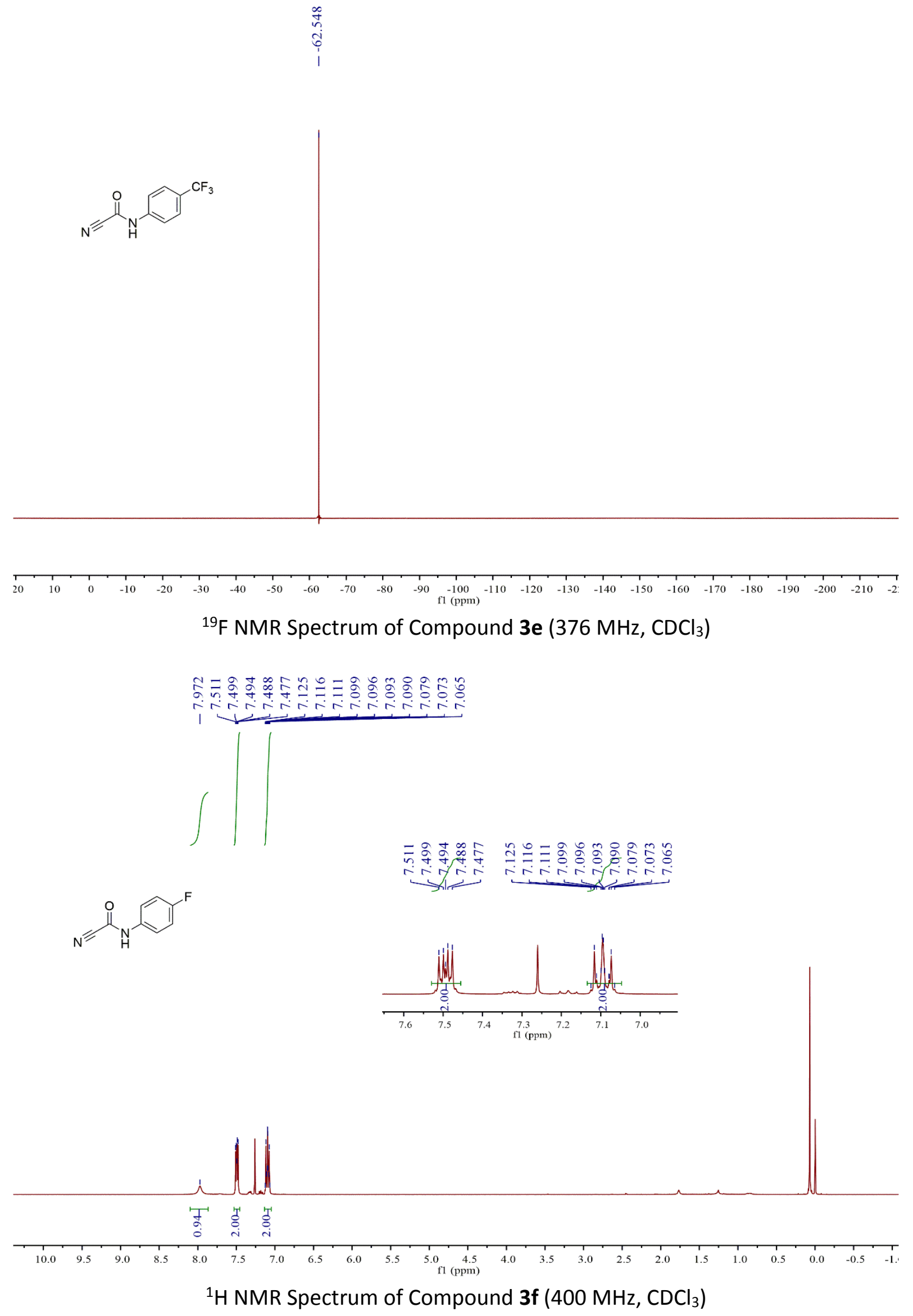



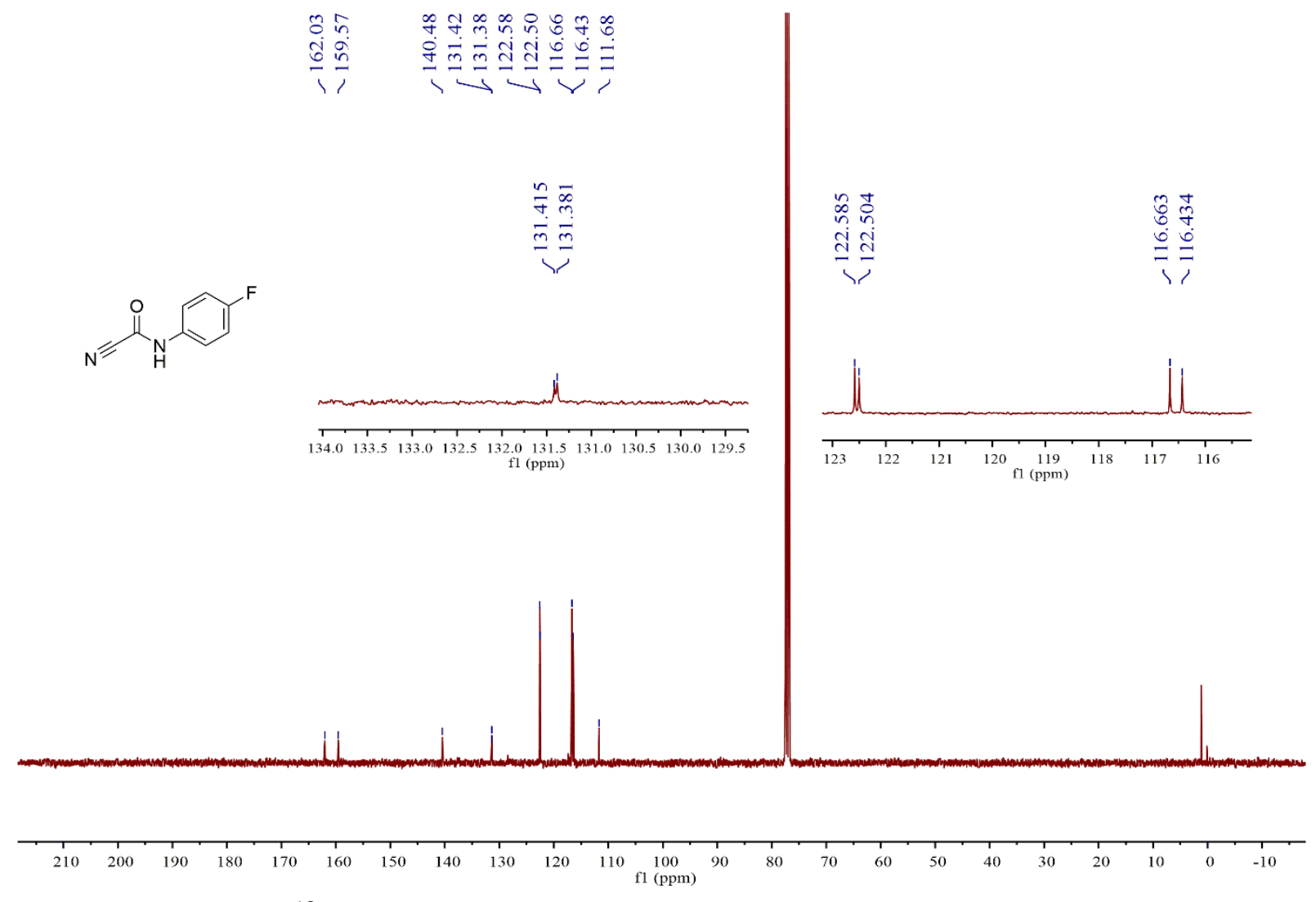

${ }^{13} \mathrm{C}$ NMR Spectrum of Compound $3 f\left(100 \mathrm{MHz}, \mathrm{CDCl}_{3}\right)$

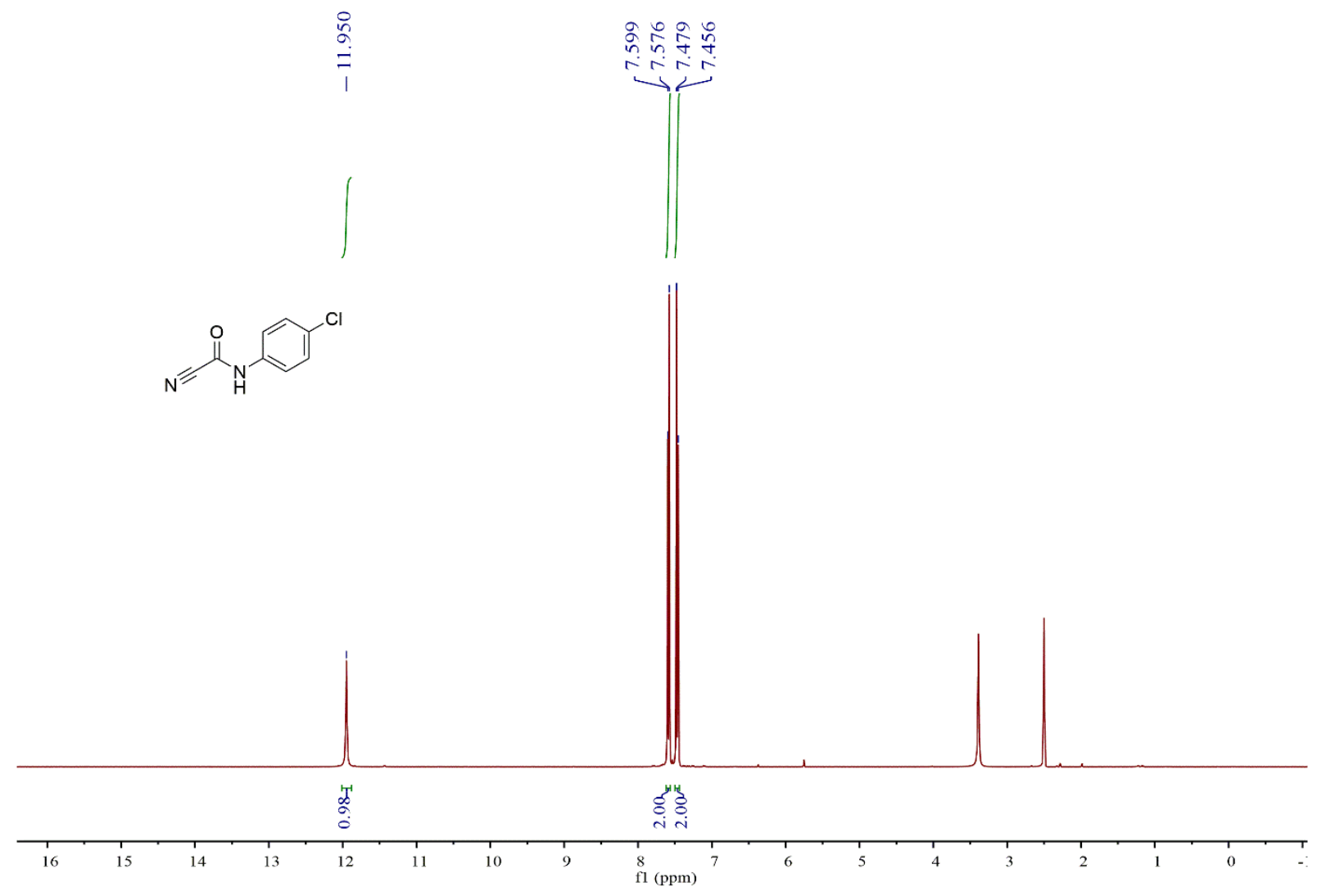

${ }^{1} \mathrm{H}$ NMR Spectrum of Compound $\mathbf{3 g}\left(400 \mathrm{MHz},\left(\mathrm{CD}_{3}\right)_{2} \mathrm{SO}\right)$ 

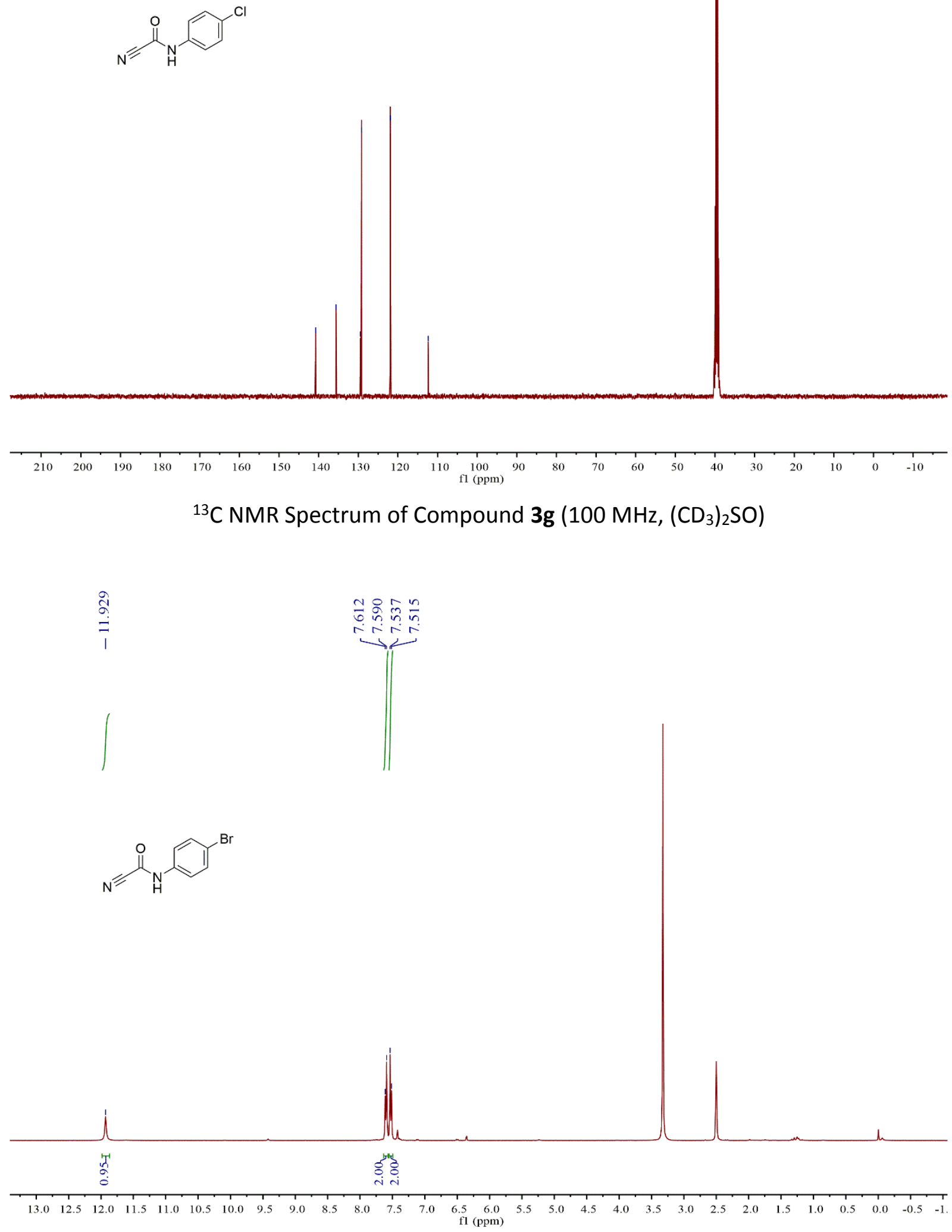

${ }^{1} \mathrm{H}$ NMR Spectrum of Compound $3 \mathrm{~h}\left(400 \mathrm{MHz},\left(\mathrm{CD}_{3}\right)_{2} \mathrm{SO}\right)$ 


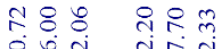

$\underset{1}{1} \frac{1}{1} \frac{1}{1}=\frac{1}{1}$
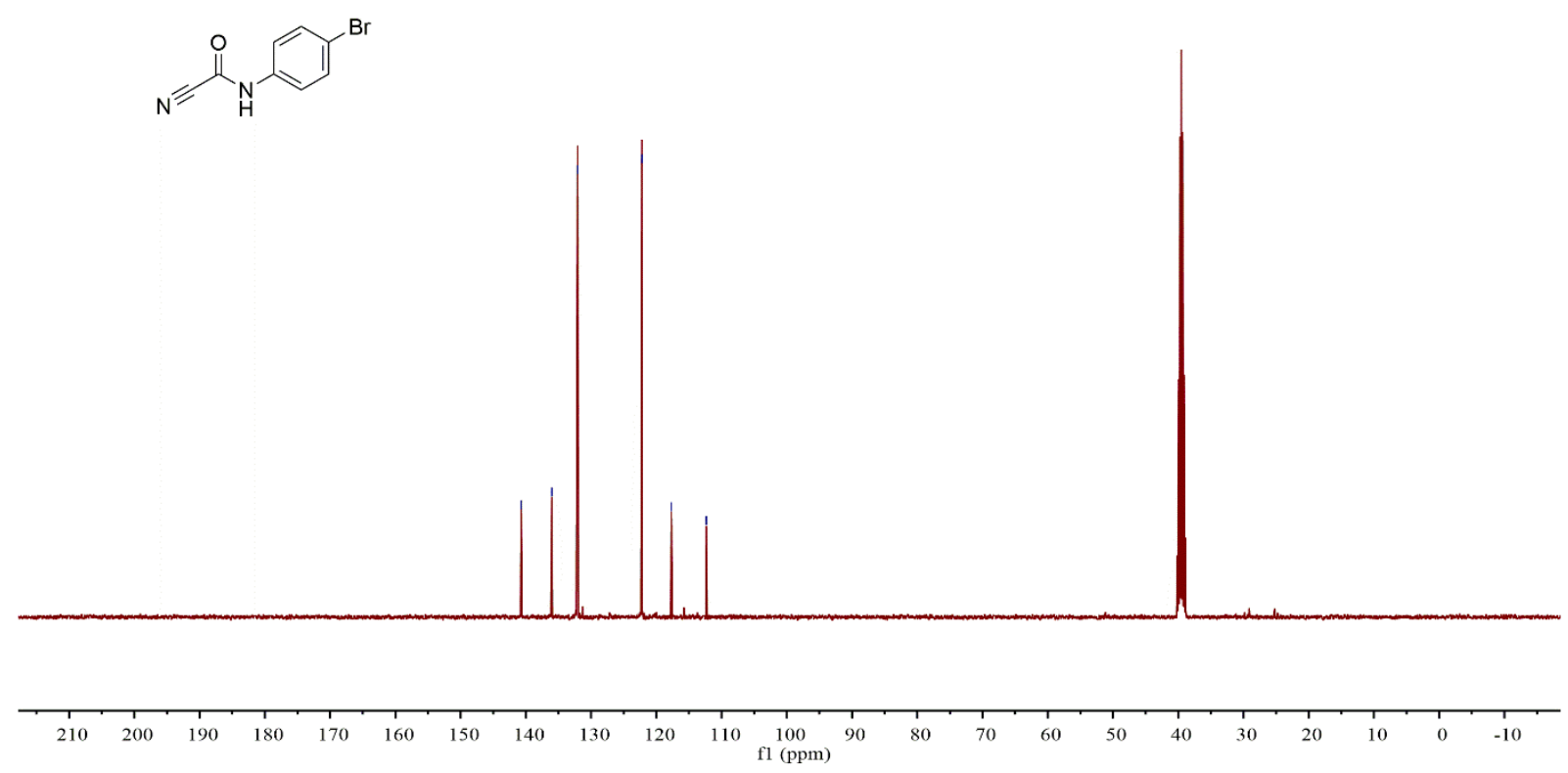

${ }^{13} \mathrm{C}$ NMR Spectrum of Compound $\mathbf{3 h}\left(100 \mathrm{MHz},\left(\mathrm{CD}_{3}\right)_{2} \mathrm{SO}\right)$

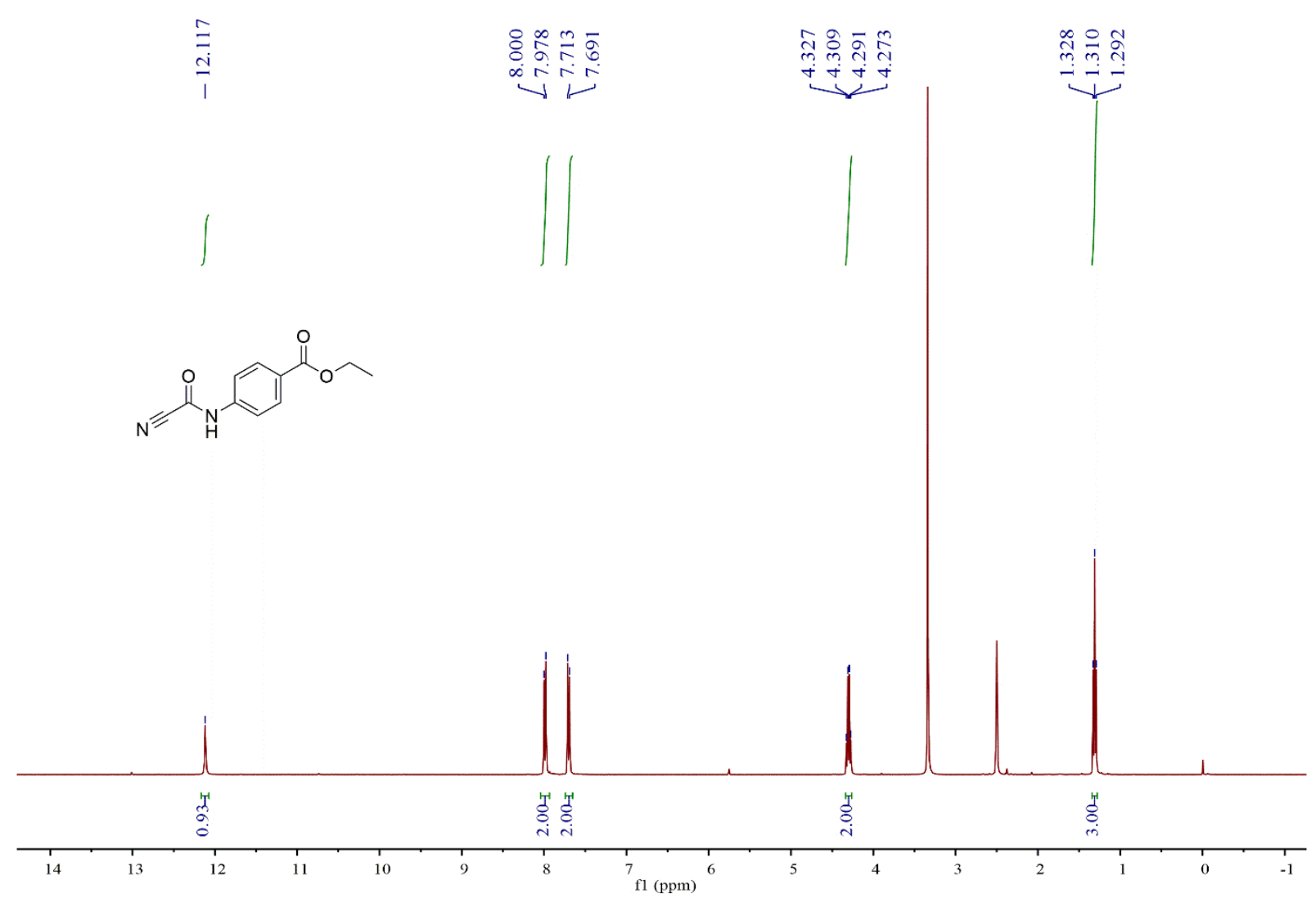

${ }^{1} \mathrm{H}$ NMR Spectrum of Compound $3 \mathbf{i}\left(400 \mathrm{MHz},\left(\mathrm{CD}_{3}\right)_{2} \mathrm{SO}\right)$ 

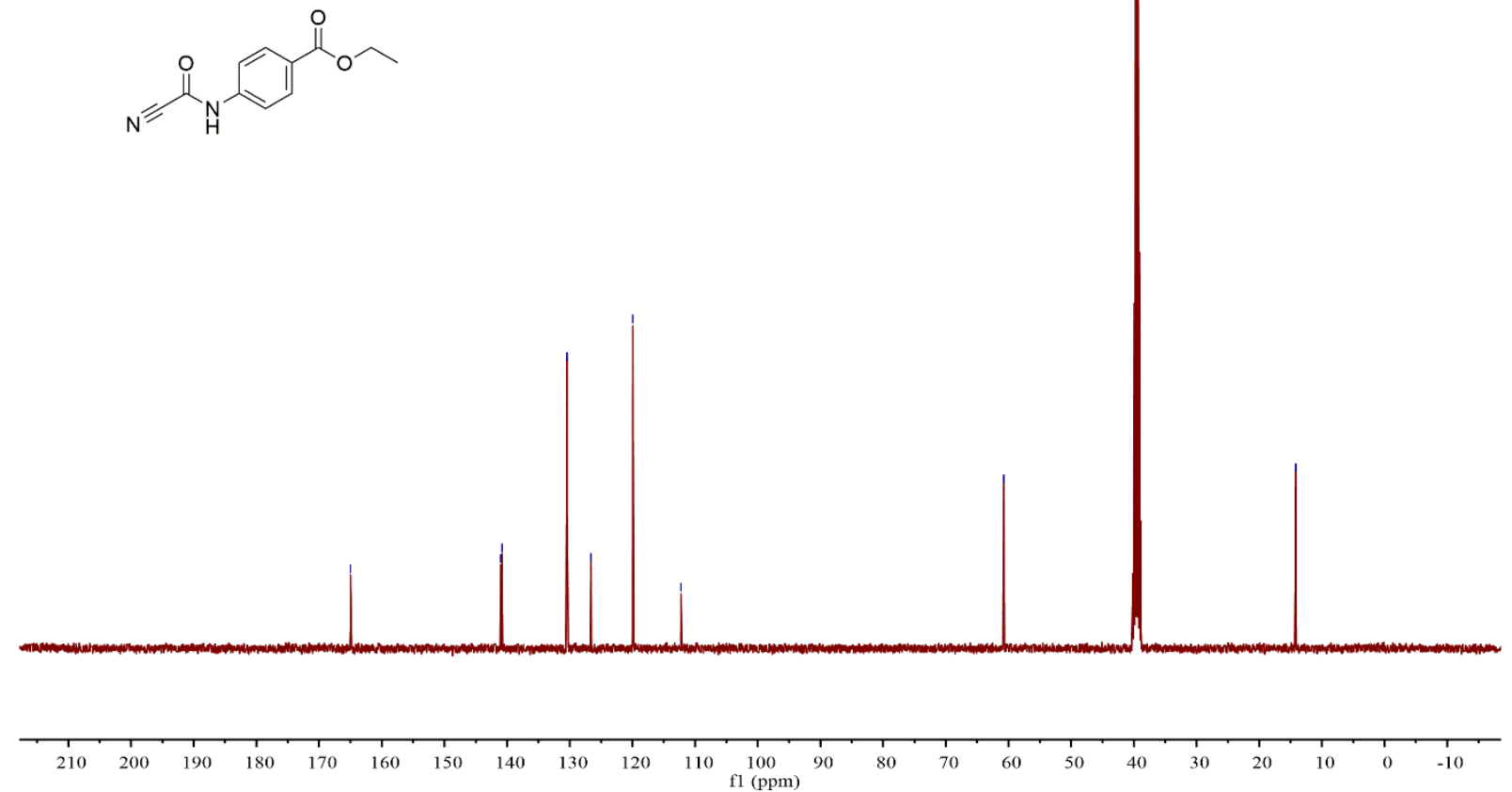

${ }^{13} \mathrm{C}$ NMR Spectrum of Compound $3 \mathbf{i}\left(100 \mathrm{MHz},\left(\mathrm{CD}_{3}\right)_{2} \mathrm{SO}\right)$

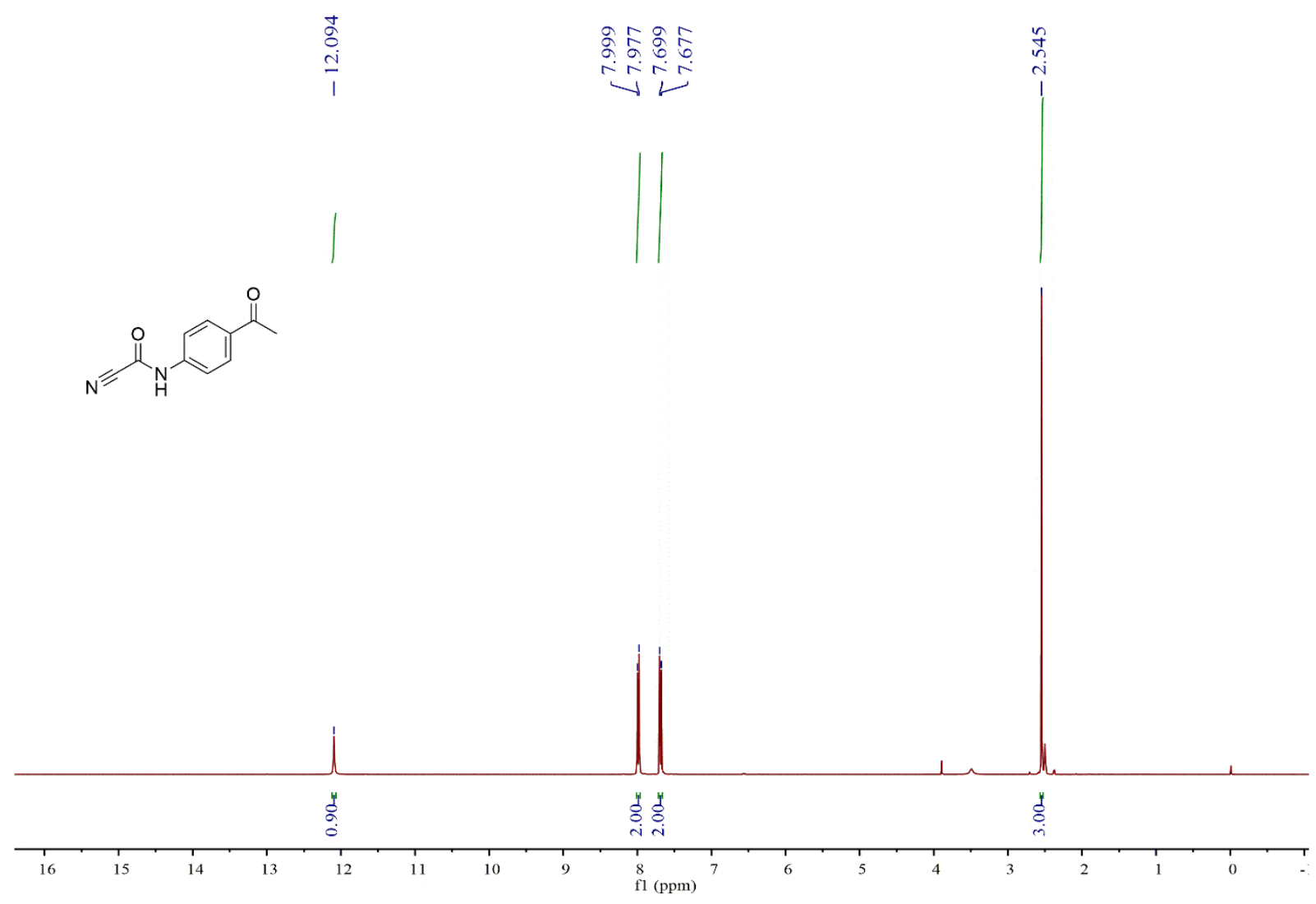

${ }^{1} \mathrm{H}$ NMR Spectrum of Compound $3 \mathbf{j}\left(400 \mathrm{MHz},\left(\mathrm{CD}_{3}\right)_{2} \mathrm{SO}\right)$ 


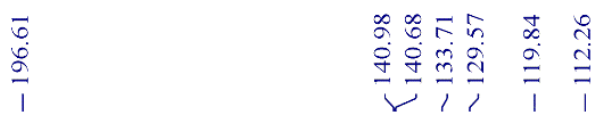

$\int_{n}$
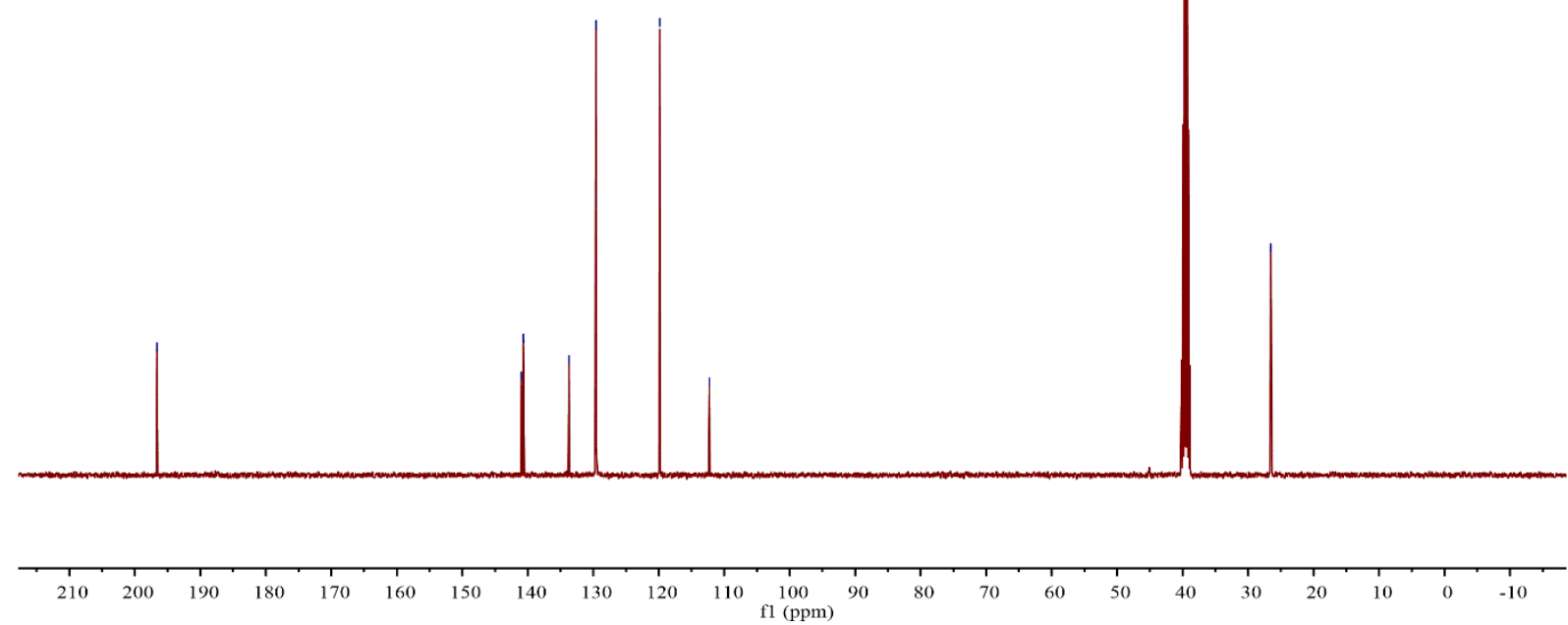

${ }^{13} \mathrm{C}$ NMR Spectrum of Compound $3 \mathbf{j}\left(100 \mathrm{MHz},\left(\mathrm{CD}_{3}\right)_{2} \mathrm{SO}\right)$

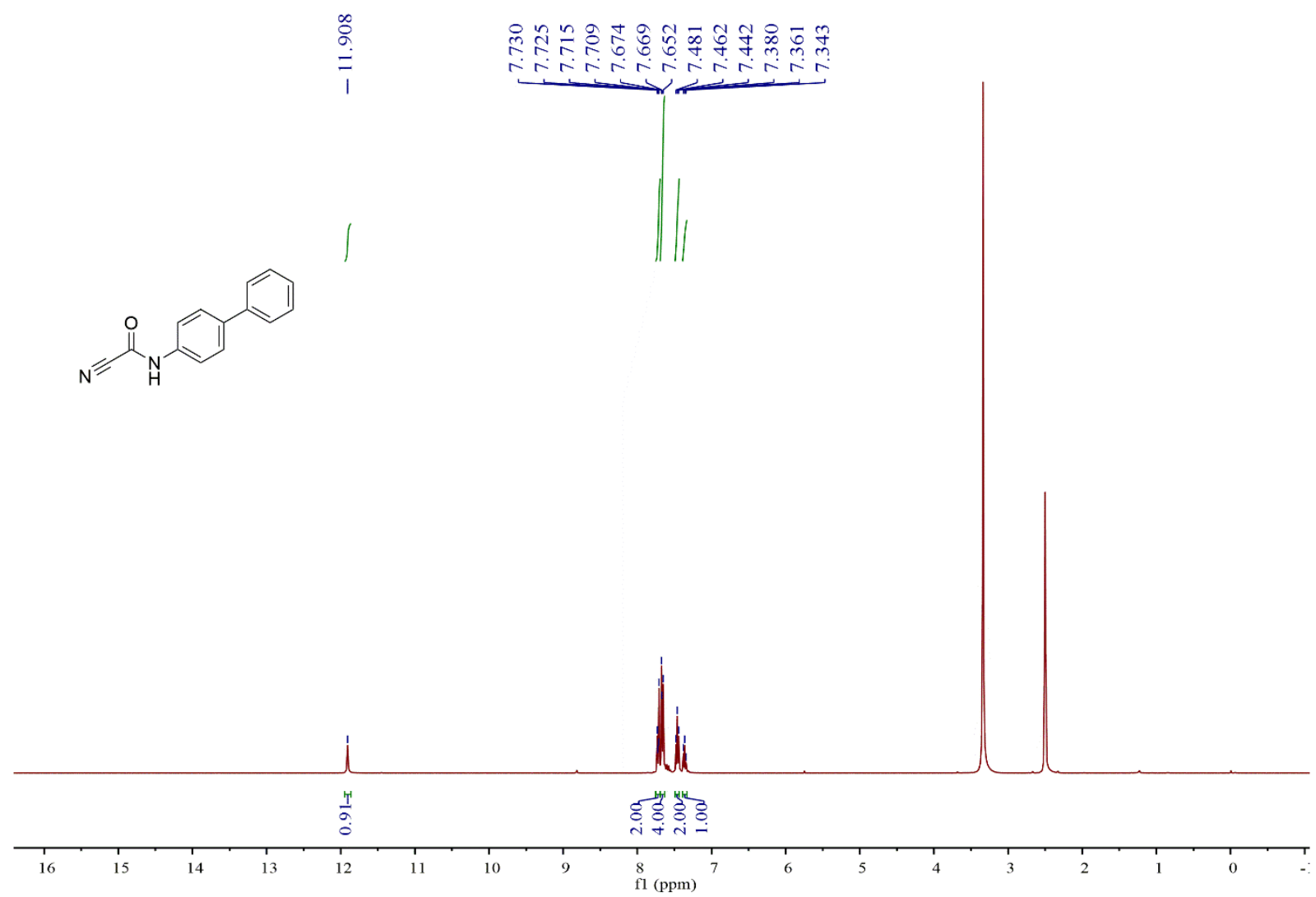

${ }^{1} \mathrm{H}$ NMR Spectrum of Compound $3 \mathbf{k}\left(400 \mathrm{MHz},\left(\mathrm{CD}_{3}\right)_{2} \mathrm{SO}\right)$ 

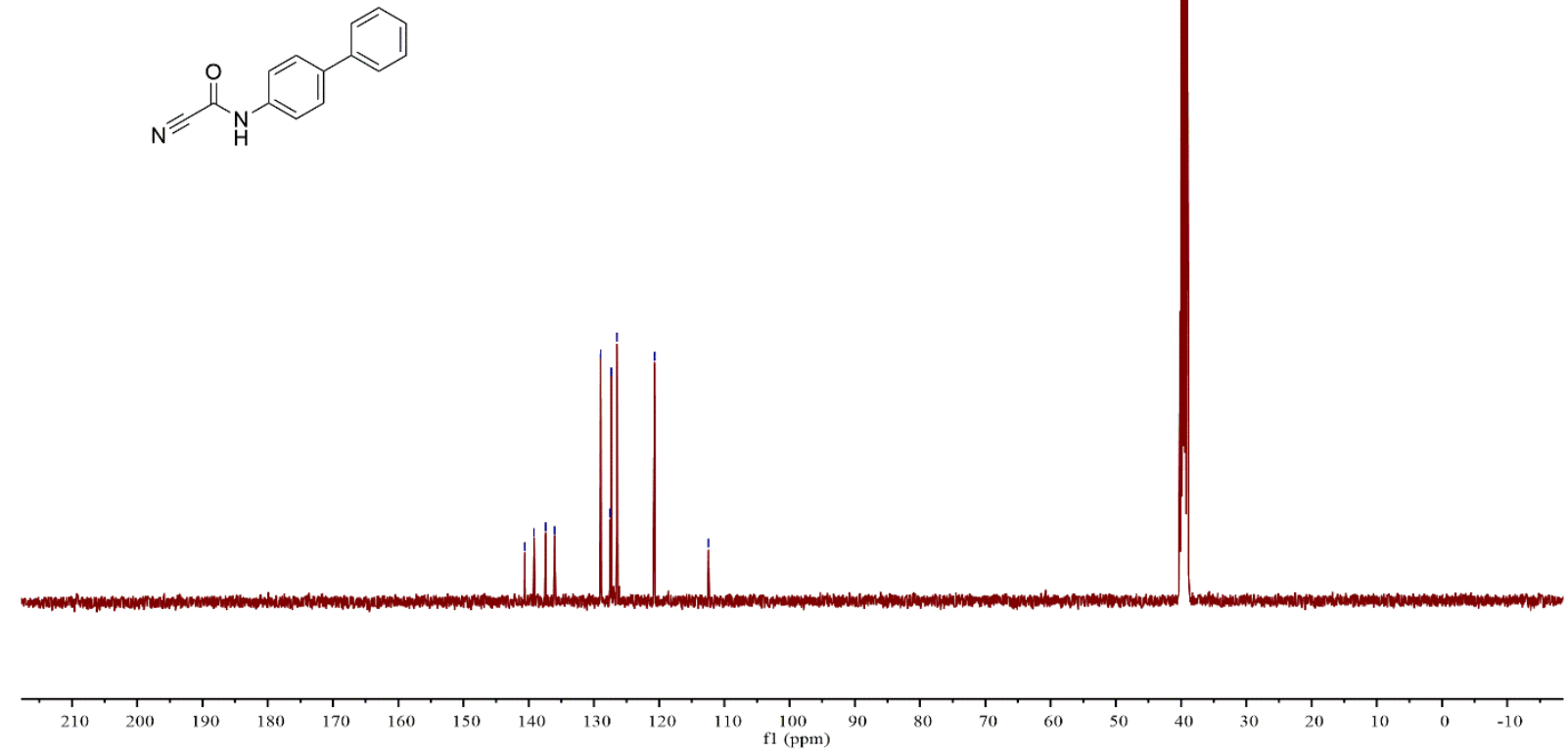

${ }^{13} \mathrm{C}$ NMR Spectrum of Compound $3 \mathbf{k}\left(100 \mathrm{MHz},\left(\mathrm{CD}_{3}\right)_{2} \mathrm{SO}\right)$

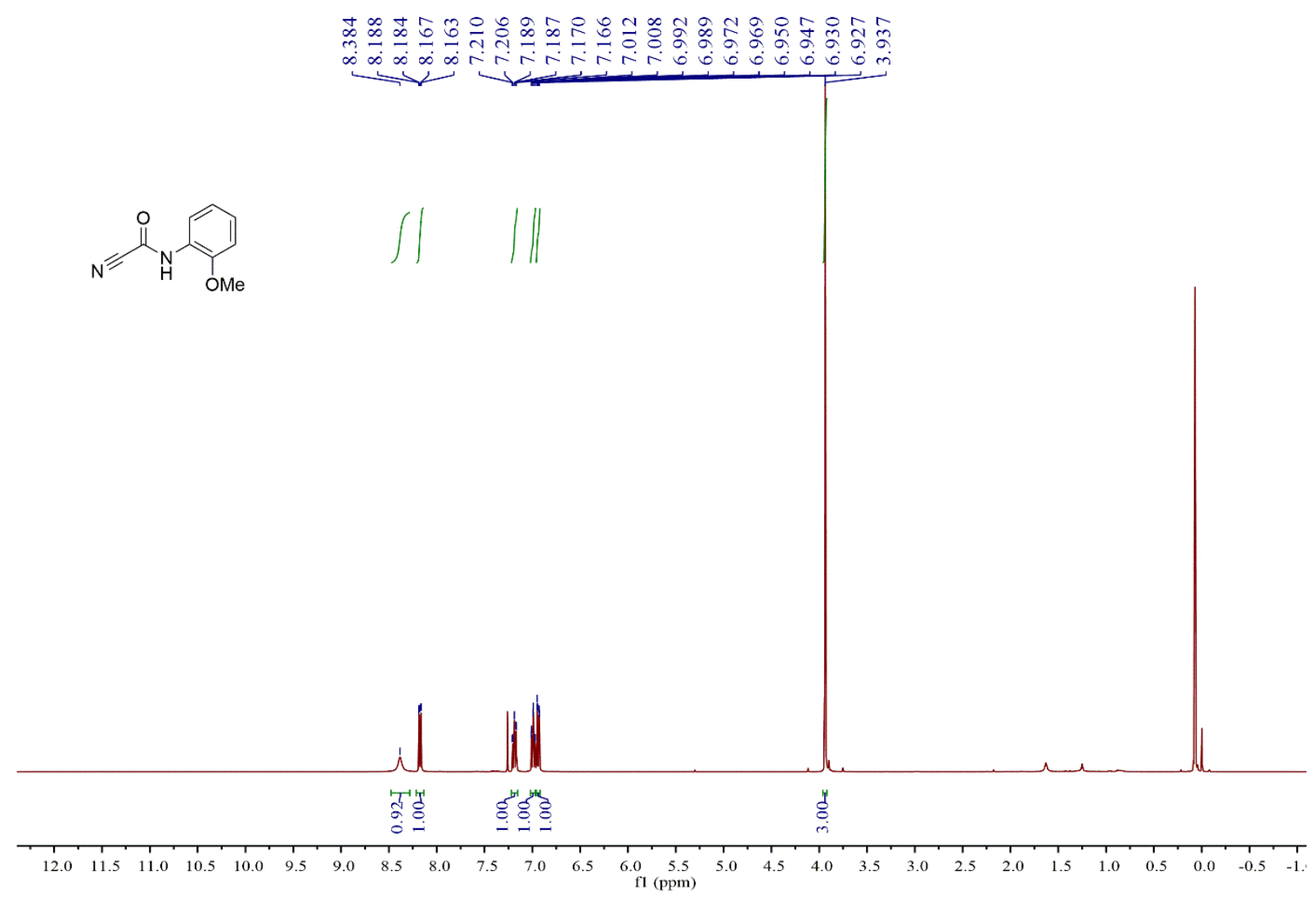

${ }^{1} \mathrm{H}$ NMR Spectrum of Compound $3 \mathrm{I}\left(400 \mathrm{MHz}, \mathrm{CDCl}_{3}\right)$ 

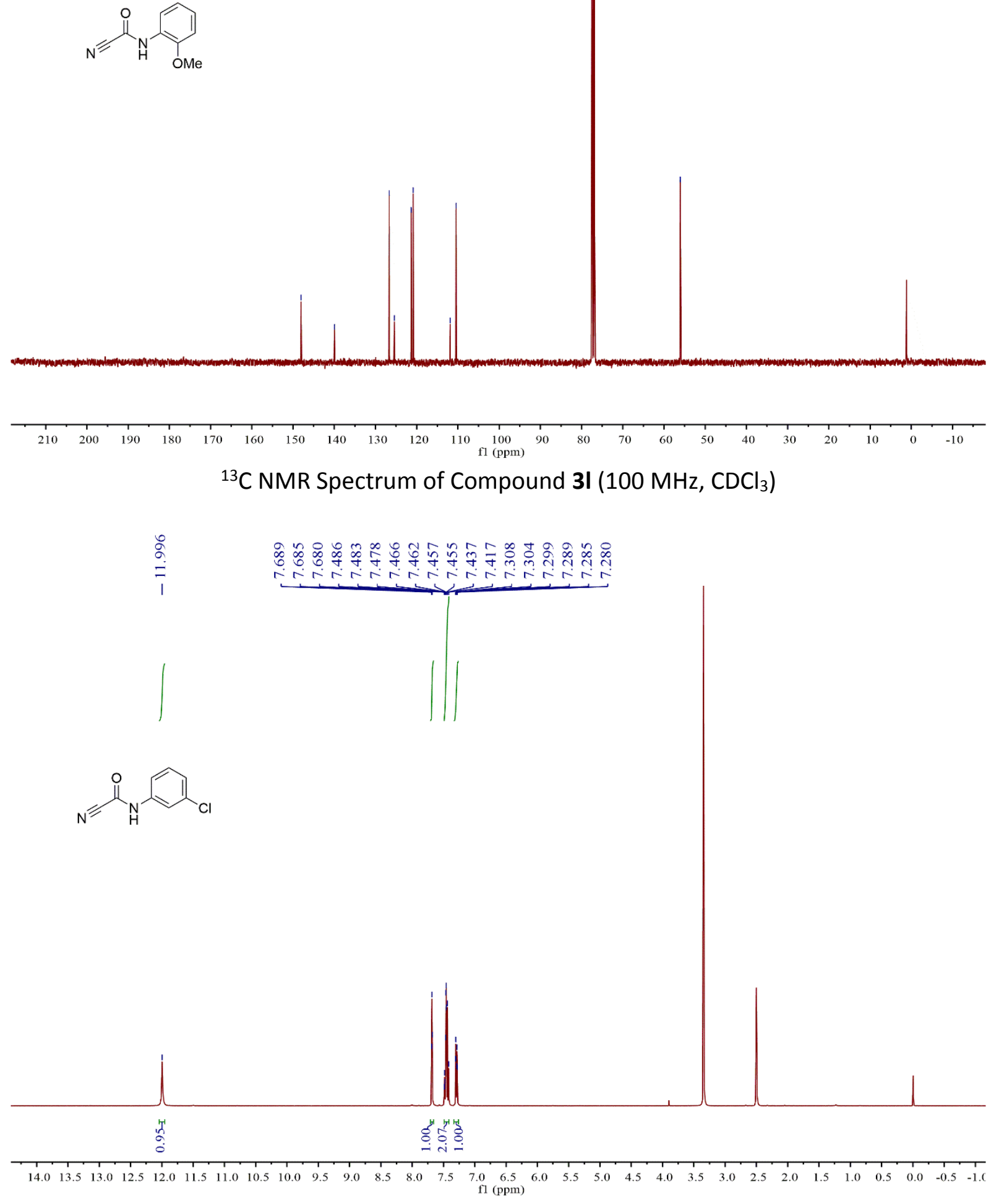

${ }^{1} \mathrm{H}$ NMR Spectrum of Compound $3 \mathrm{~m}\left(400 \mathrm{MHz},\left(\mathrm{CD}_{3}\right)_{2} \mathrm{SO}\right)$ 


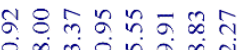

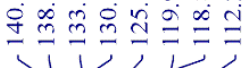

$n=\frac{H}{C}$
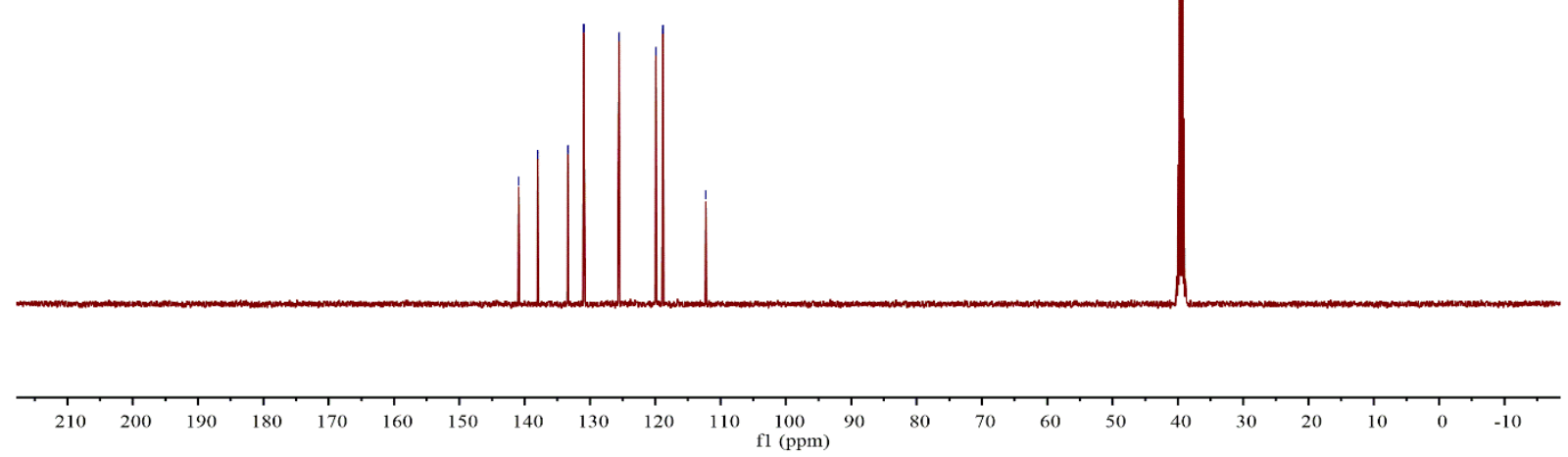

${ }^{13} \mathrm{C}$ NMR Spectrum of Compound $3 \mathrm{~m}\left(100 \mathrm{MHz},\left(\mathrm{CD}_{3}\right)_{2} \mathrm{SO}\right)$

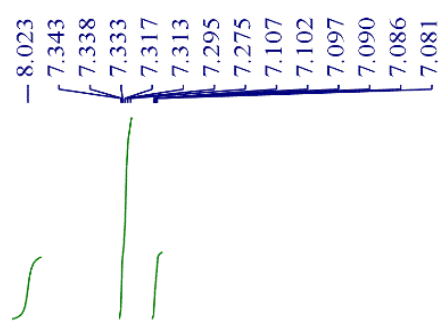

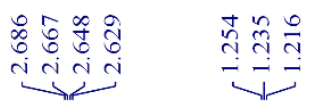

$\mathrm{N}=$

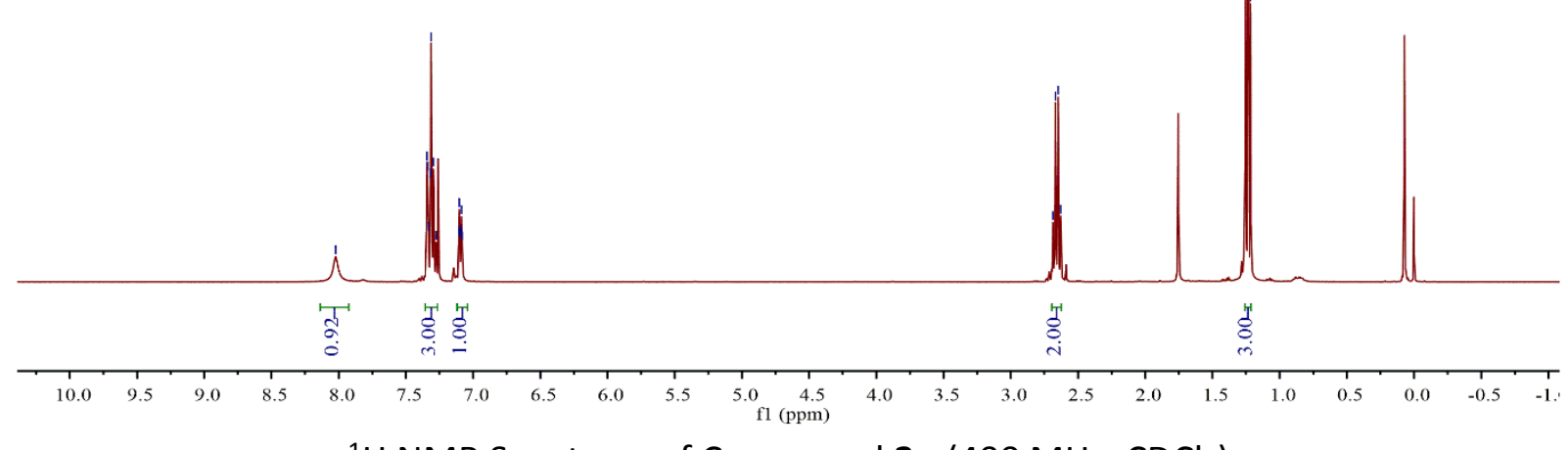

${ }^{1} \mathrm{H}$ NMR Spectrum of Compound $3 \mathbf{n}\left(400 \mathrm{MHz}, \mathrm{CDCl}_{3}\right)$ 


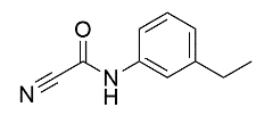
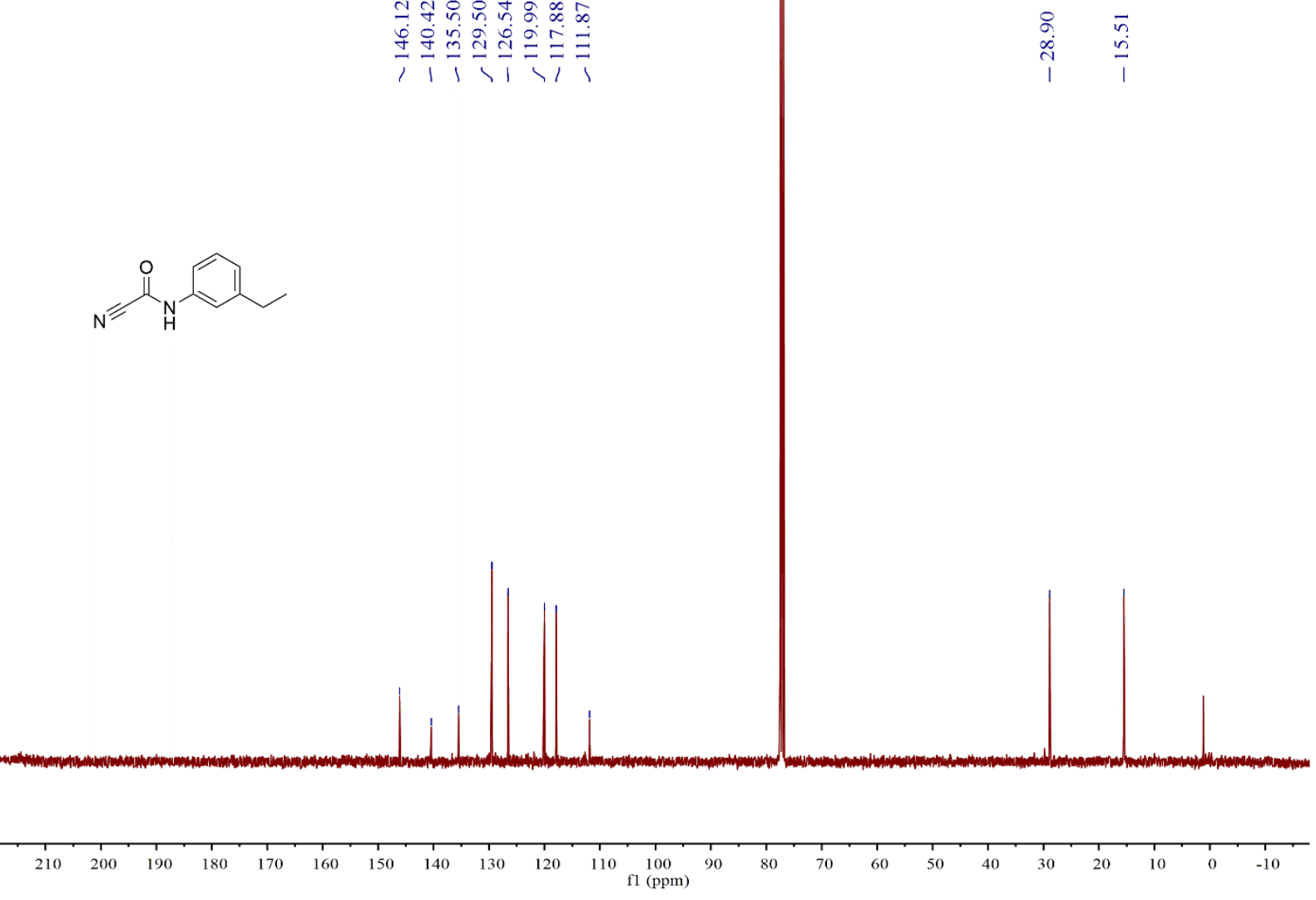

${ }^{13} \mathrm{C}$ NMR Spectrum of Compound $\left.3 \mathrm{n}\left(100 \mathrm{MHz}^{\mathrm{CDCl}}\right)_{3}\right)$

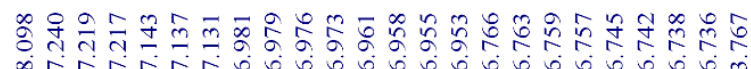

o.

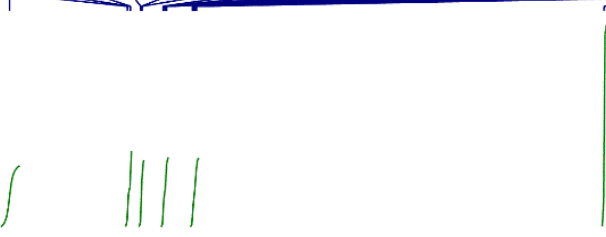

$n=\frac{H}{n}$

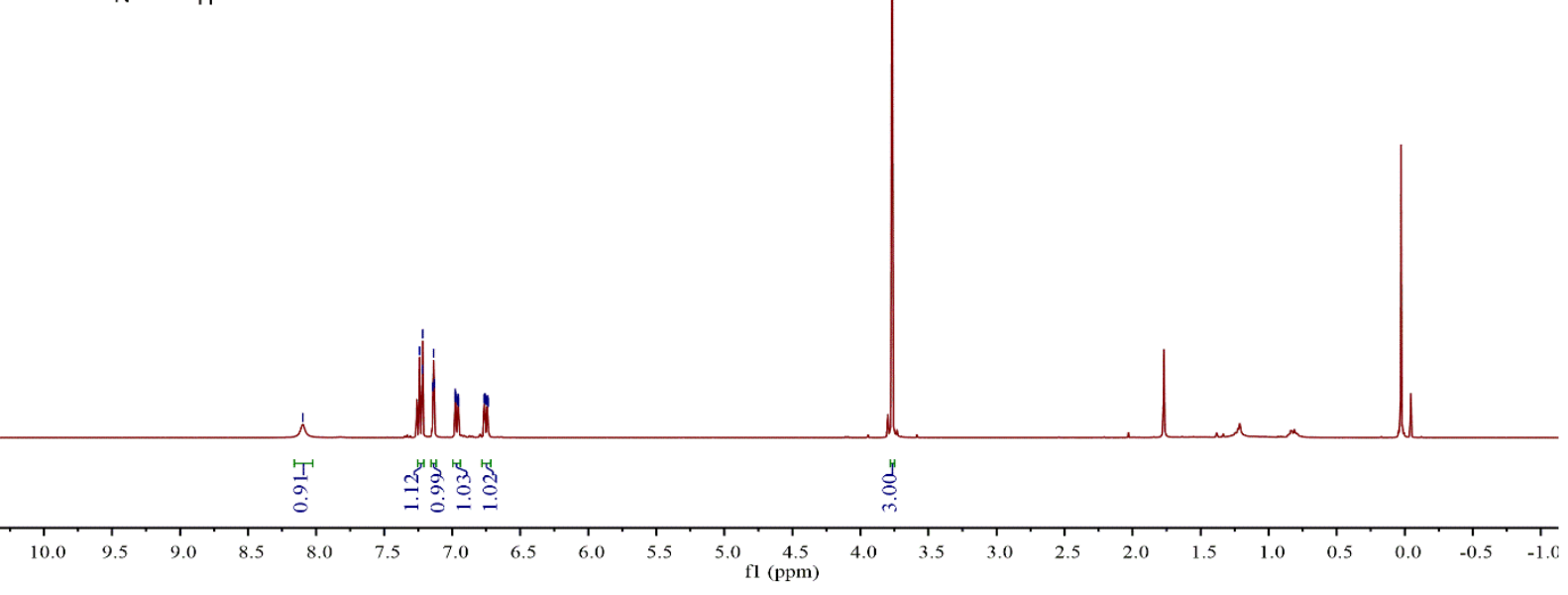

${ }^{1} \mathrm{H}$ NMR Spectrum of Compound $30\left(400 \mathrm{MHz}, \mathrm{CDCl}_{3}\right)$ 

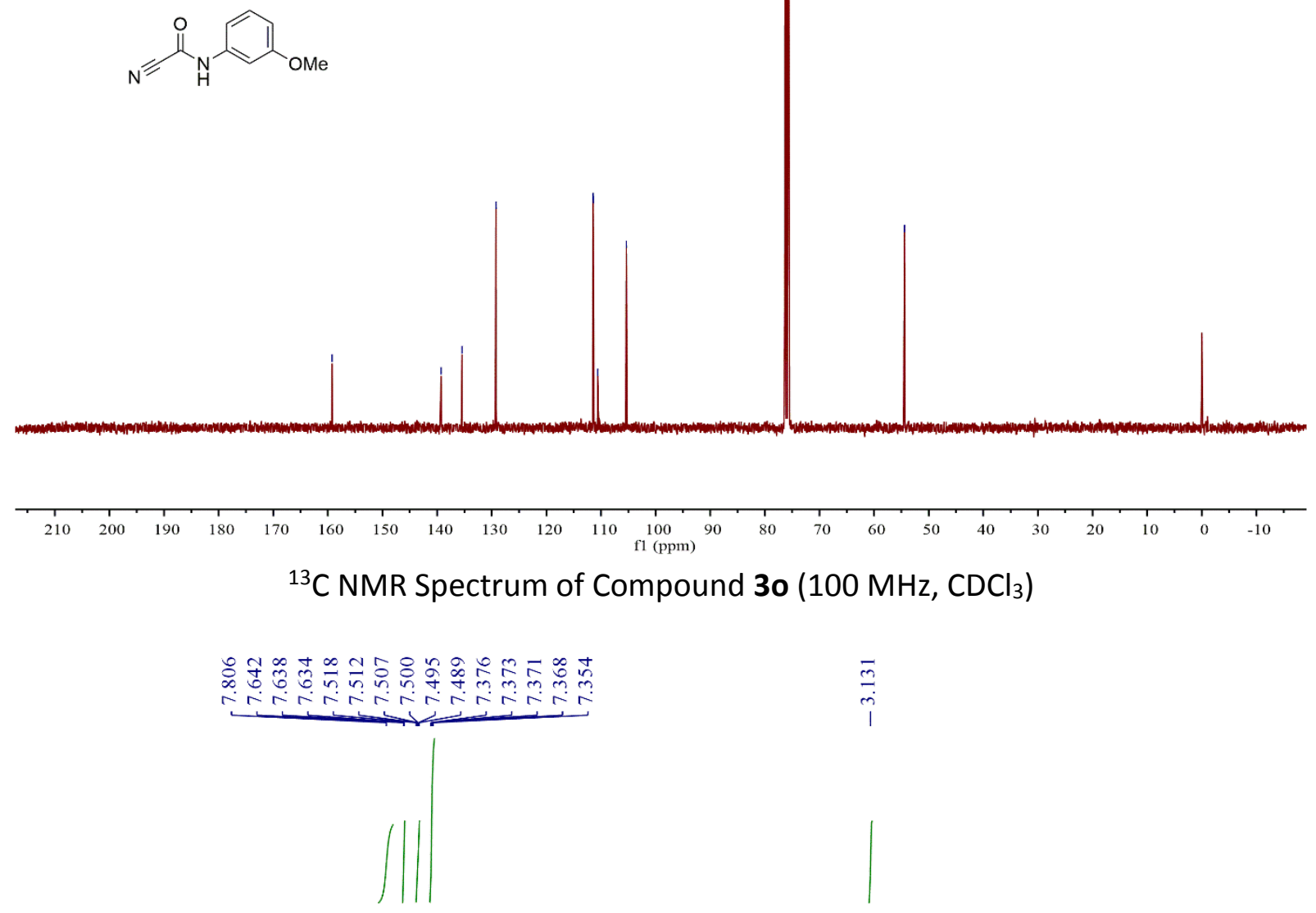<smiles>C=C1C=C2NC(C1)CC2=C</smiles>

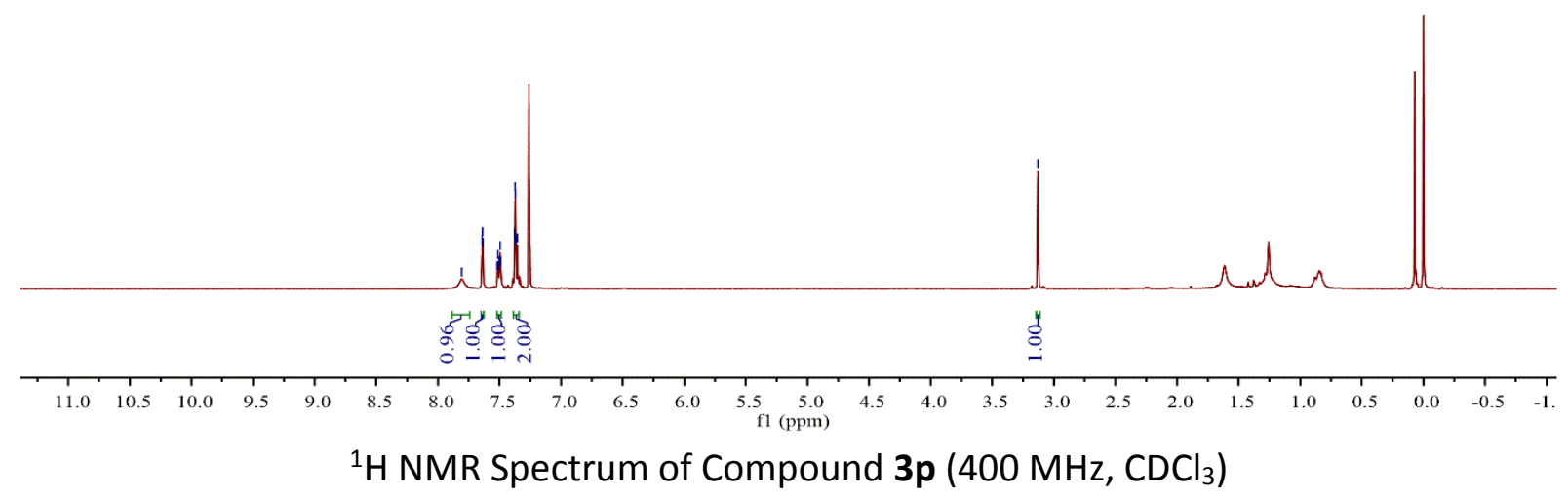



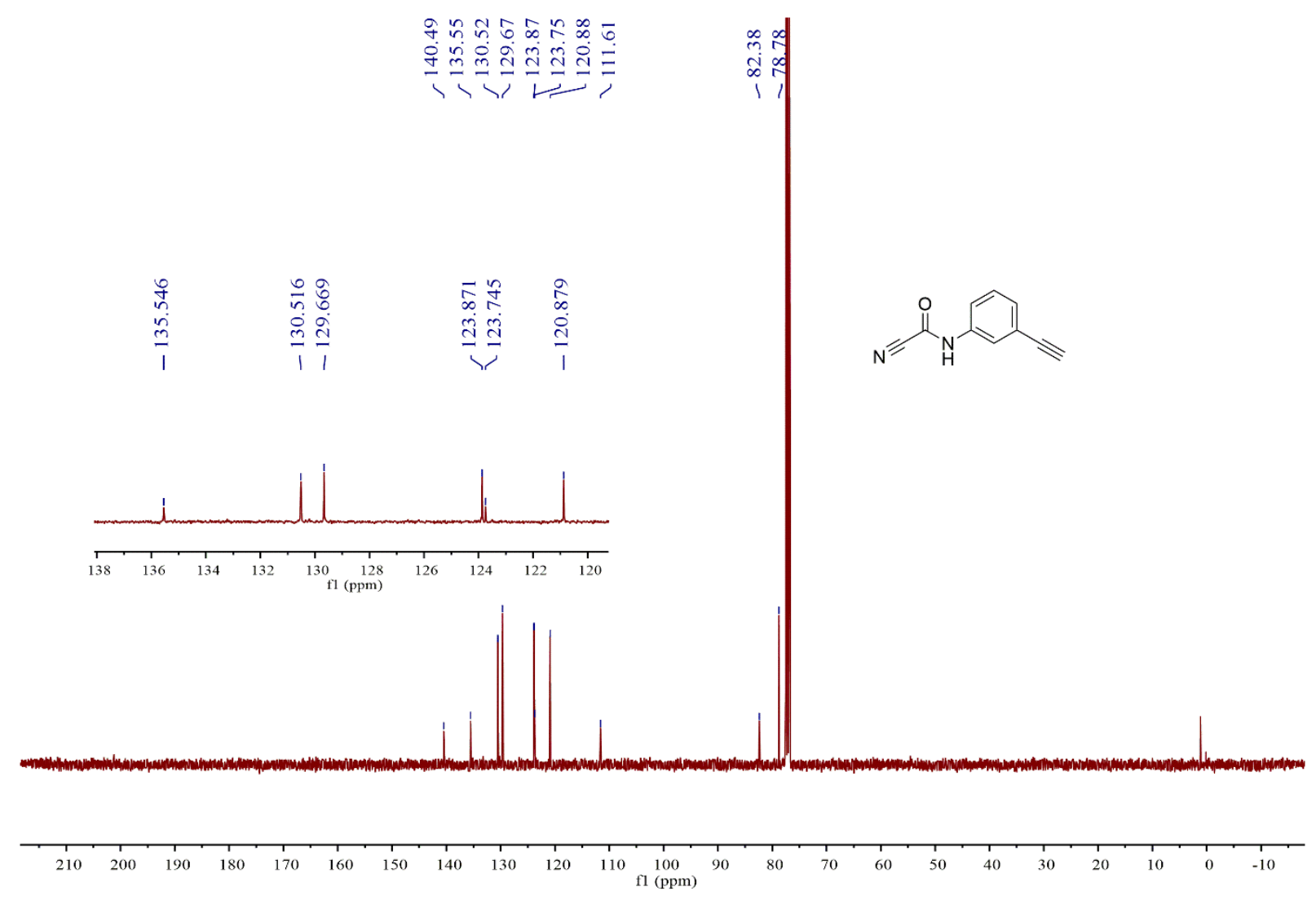

${ }^{13} \mathrm{C}$ NMR Spectrum of Compound $3 p\left(100 \mathrm{MHz} \mathrm{CDCl}_{3}\right.$ )

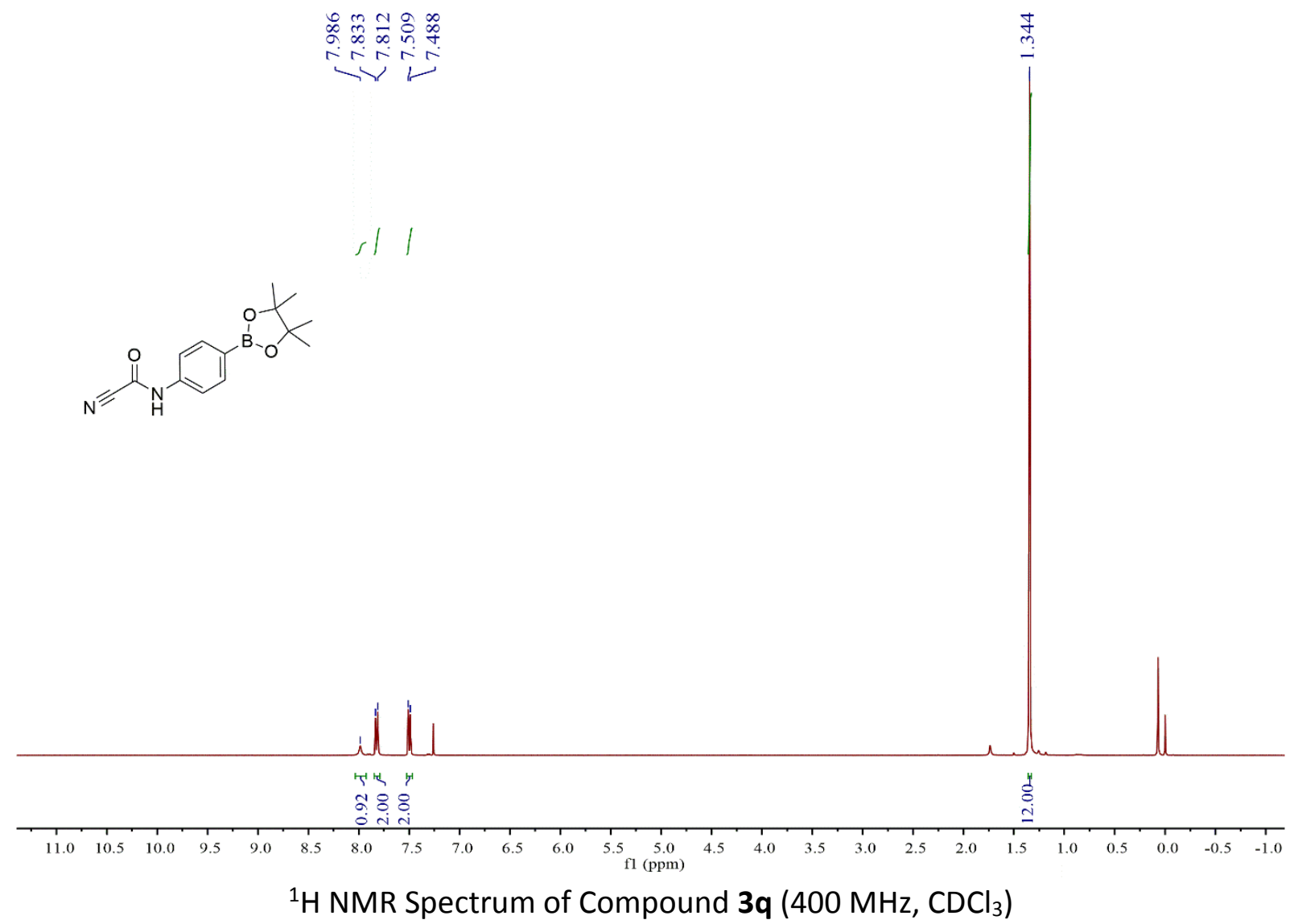



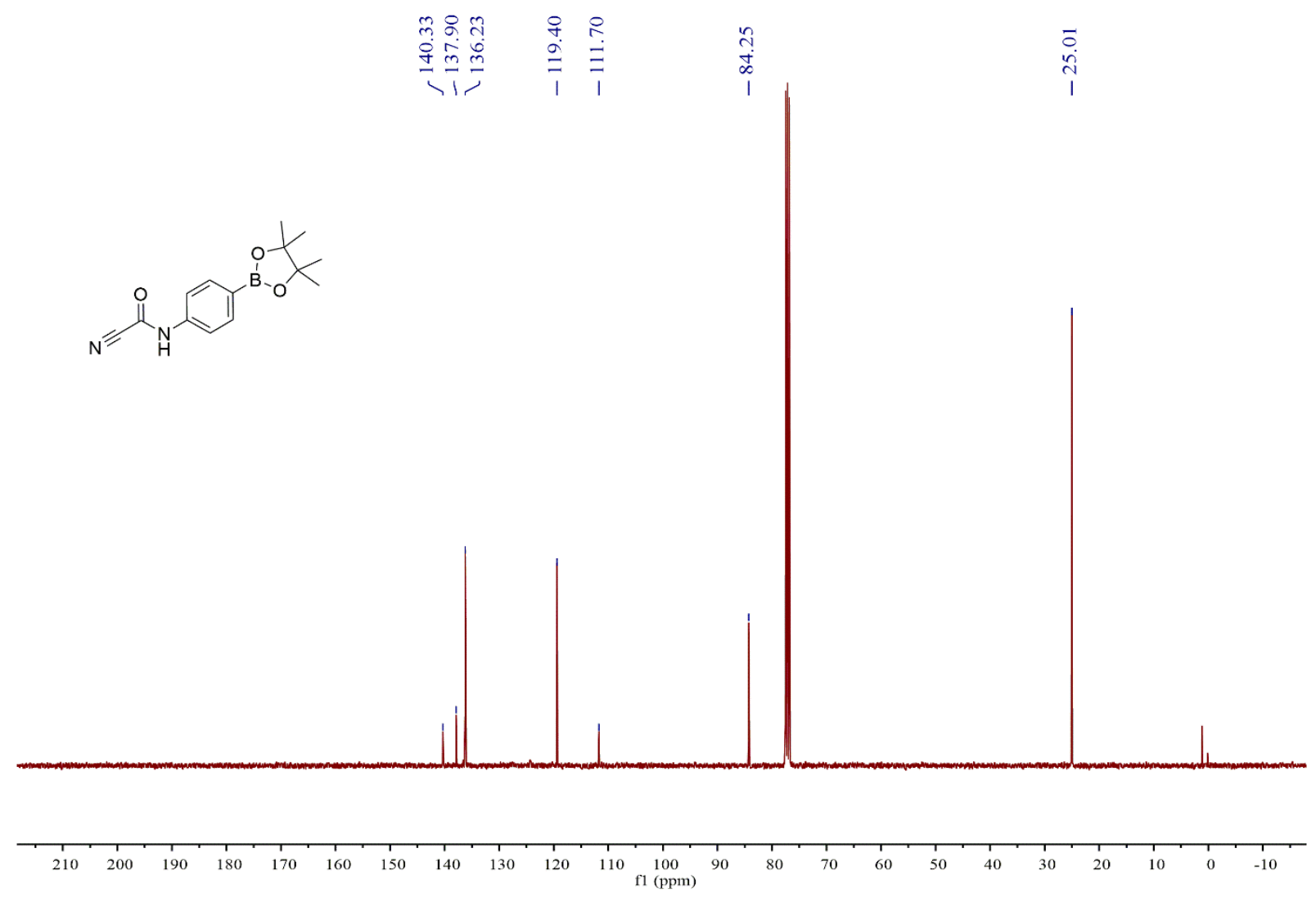

${ }^{13} \mathrm{C}$ NMR Spectrum of Compound $\mathbf{3 q}\left(100 \mathrm{MHz}^{\mathrm{CDCl}} \mathrm{CD}_{3}\right)$

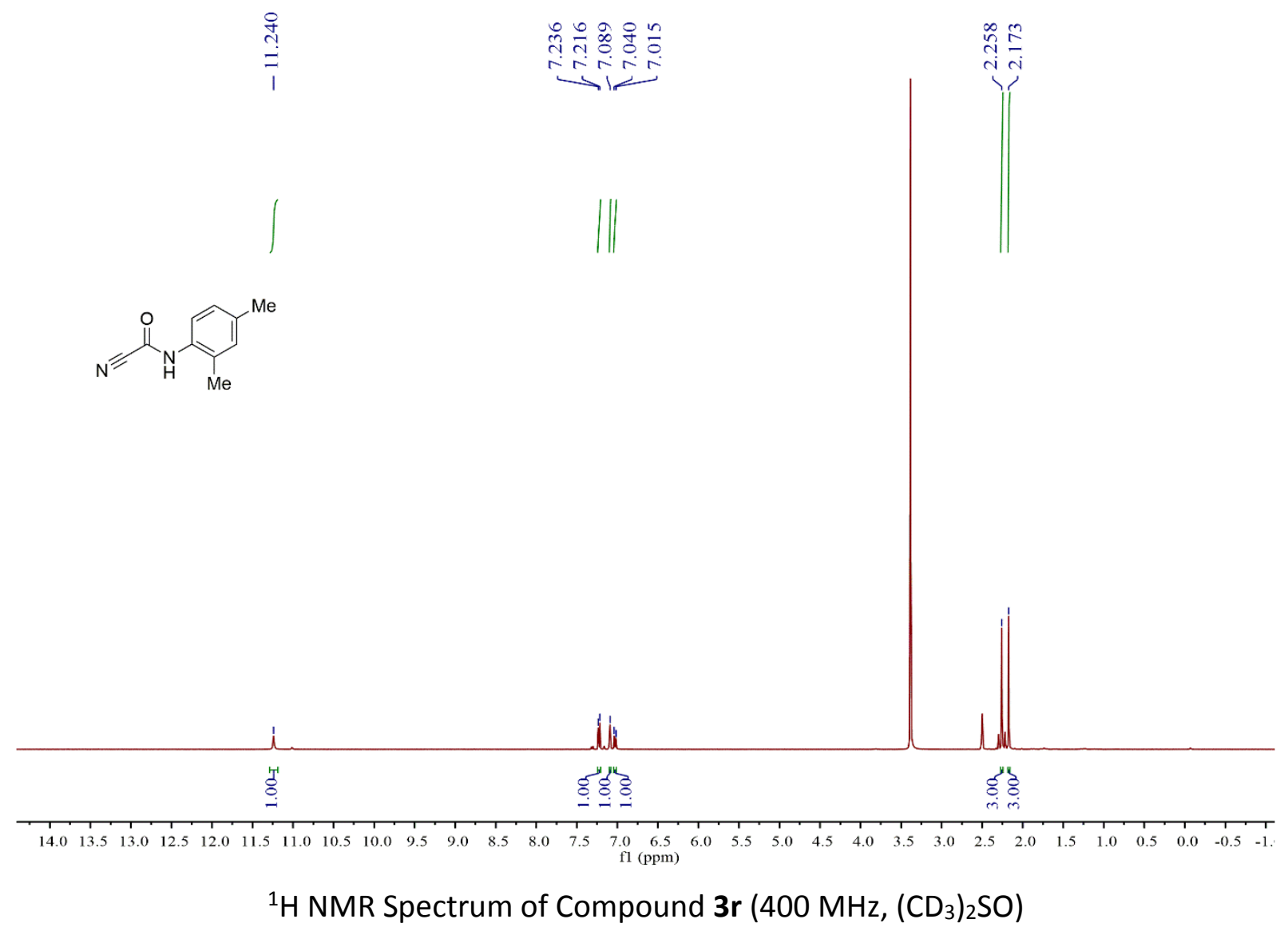


${ }_{N}$
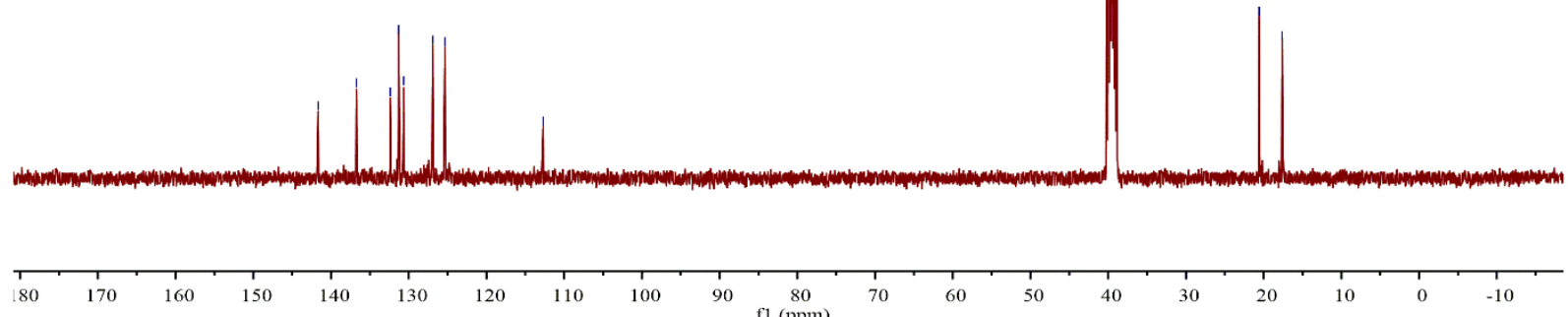

${ }^{13} \mathrm{C}$ NMR Spectrum of Compound $3 r\left(100 \mathrm{MHz},\left(\mathrm{CD}_{3}\right)_{2} \mathrm{SO}\right)$
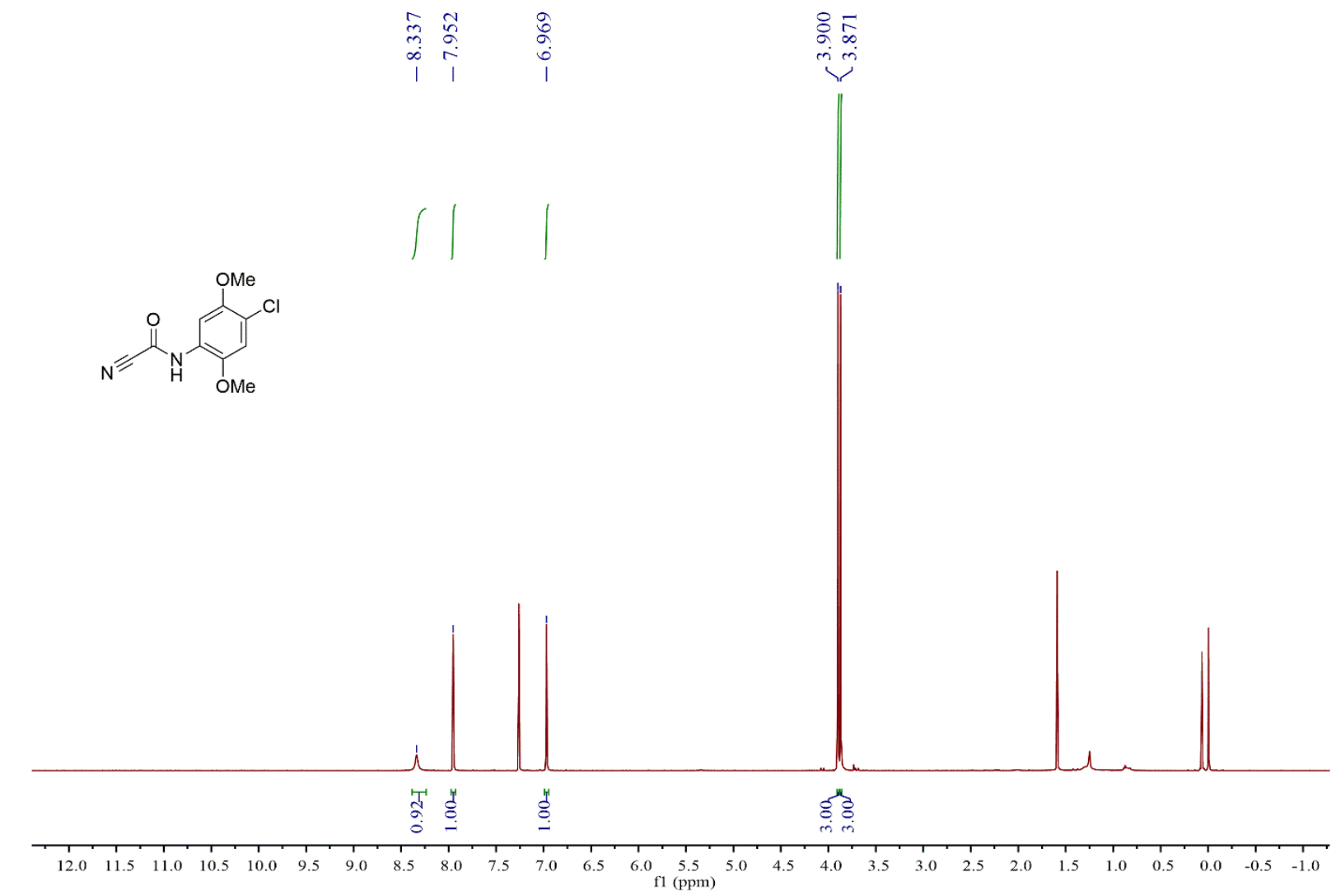

${ }^{1} \mathrm{H}$ NMR Spectrum of Compound $3 \mathbf{s}\left(400 \mathrm{MHz}, \mathrm{CDCl}_{3}\right)$ 

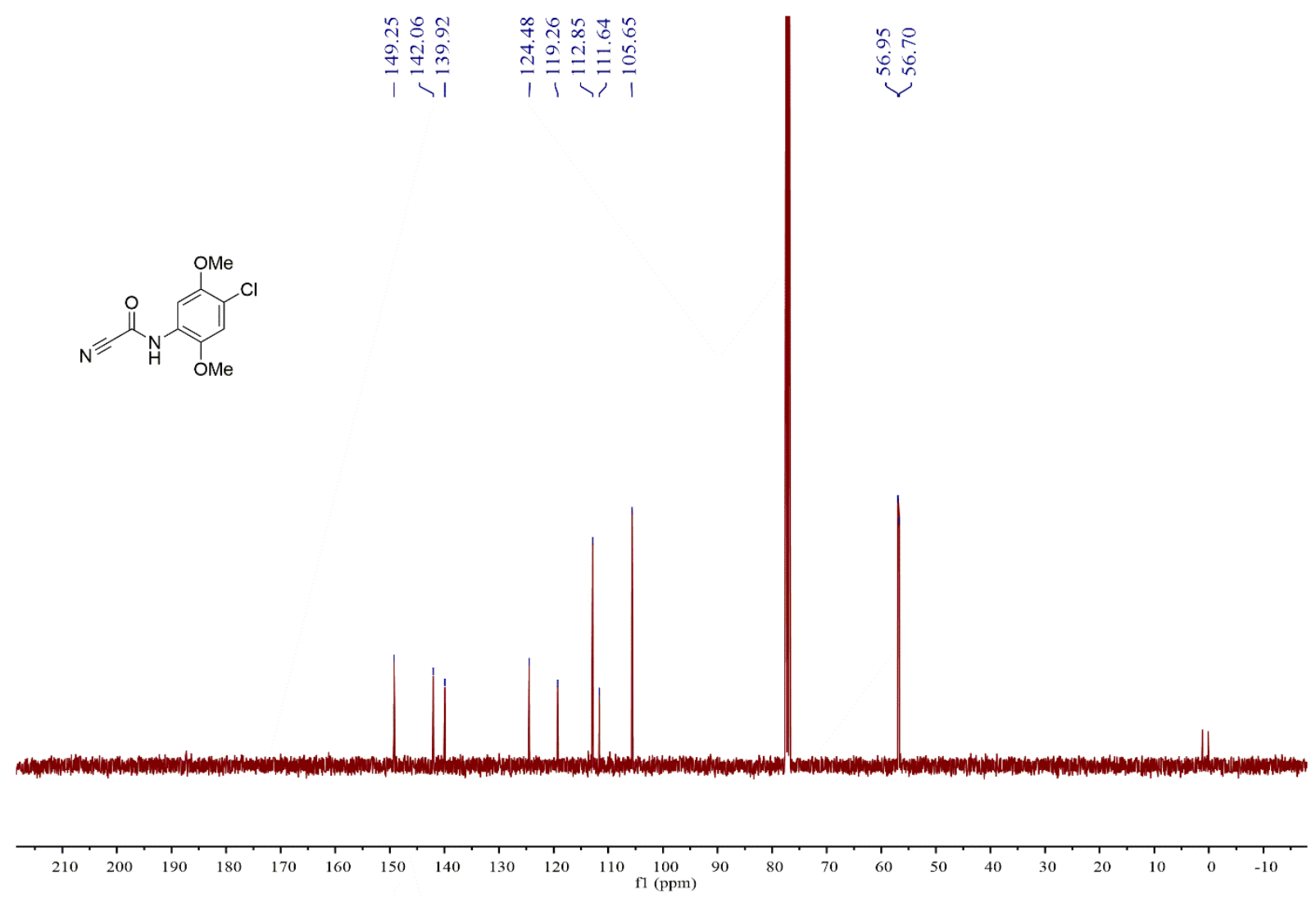

${ }^{13} \mathrm{C}$ NMR Spectrum of Compound 3s $\left(100 \mathrm{MHz}, \mathrm{CDCl}_{3}\right)$
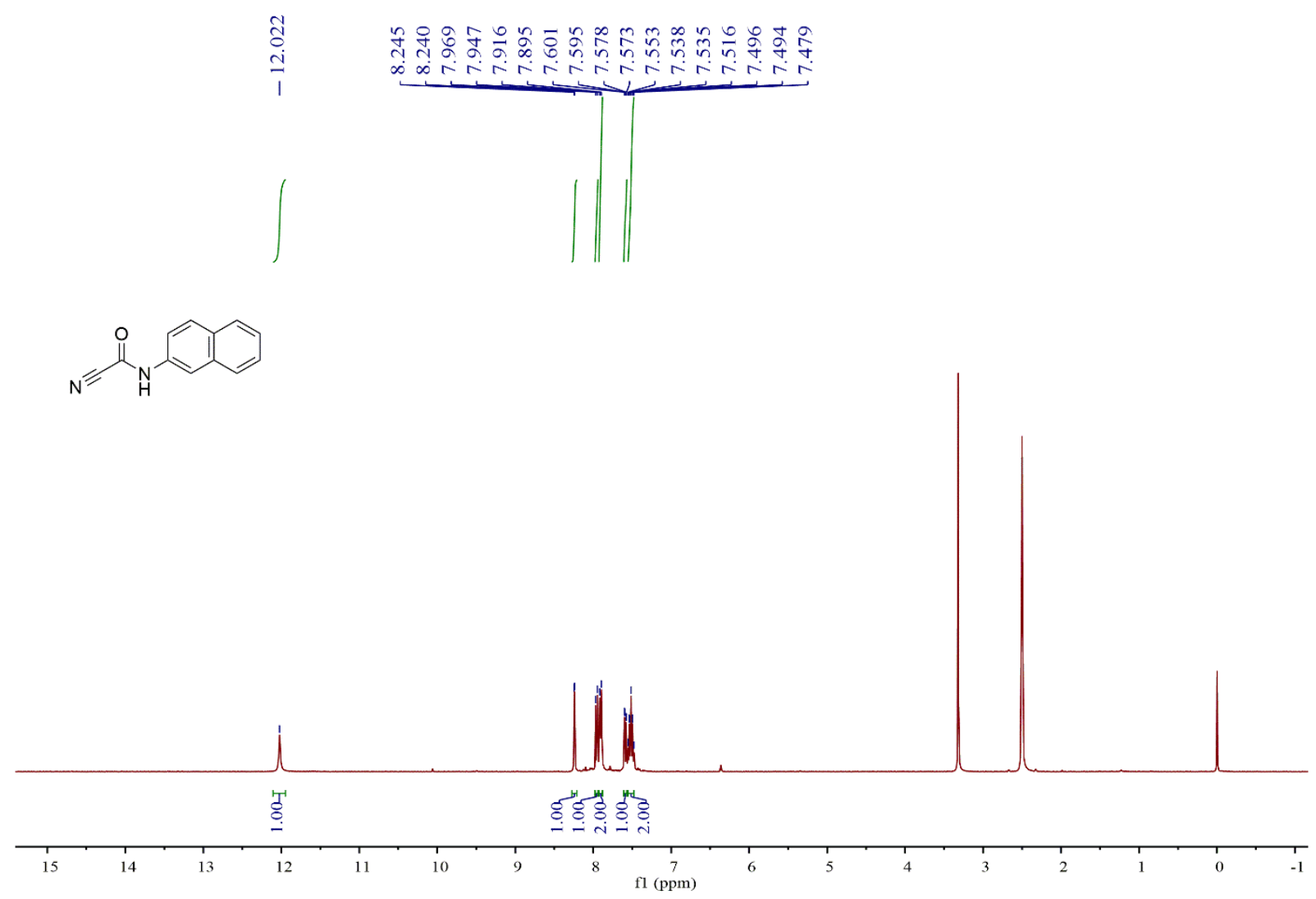

${ }^{1} \mathrm{H}$ NMR Spectrum of Compound $3 t\left(400 \mathrm{MHz},\left(\mathrm{CD}_{3}\right)_{2} \mathrm{SO}\right)$ 

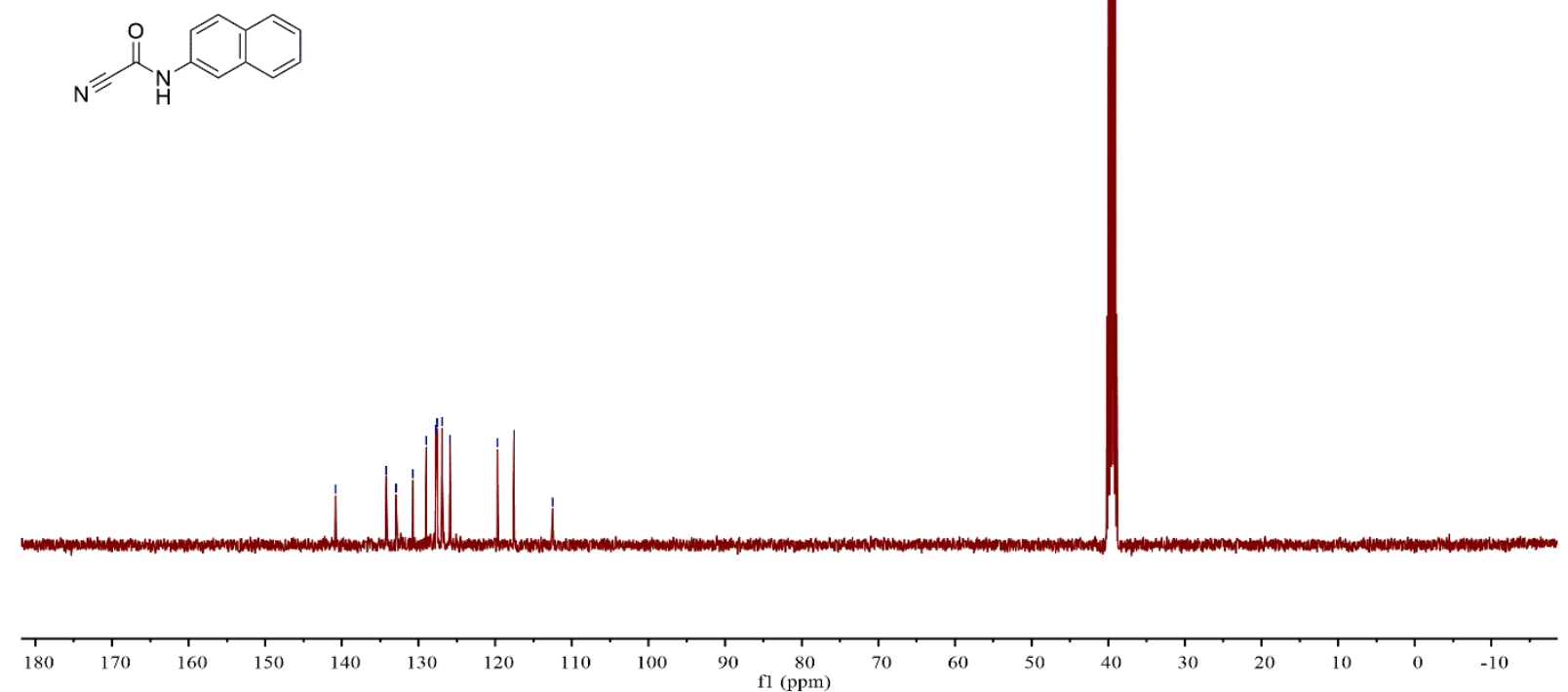

${ }^{13} \mathrm{C}$ NMR Spectrum of Compound 3t $\left(100 \mathrm{MHz},\left(\mathrm{CD}_{3}\right)_{2} \mathrm{SO}\right)$

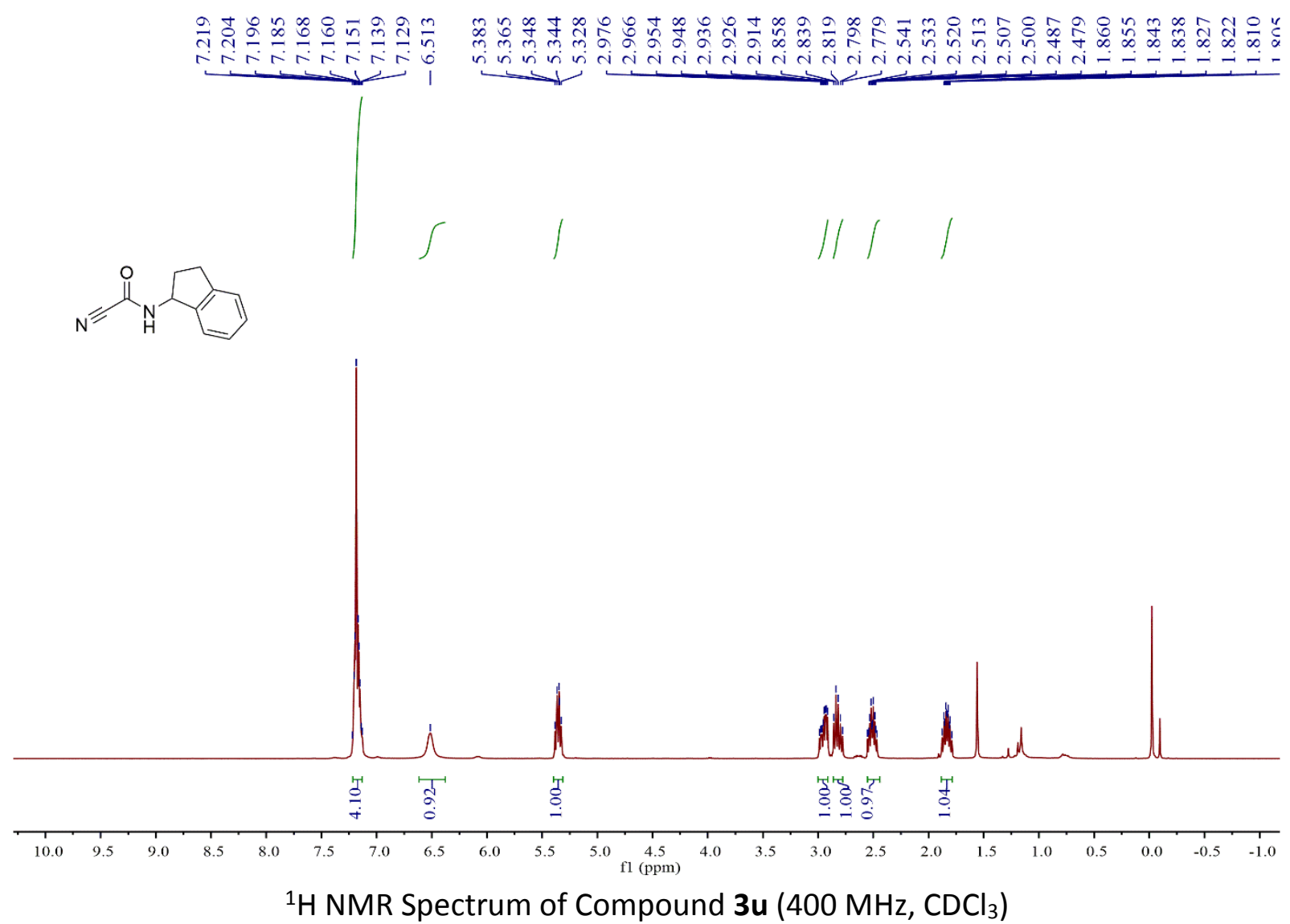


N
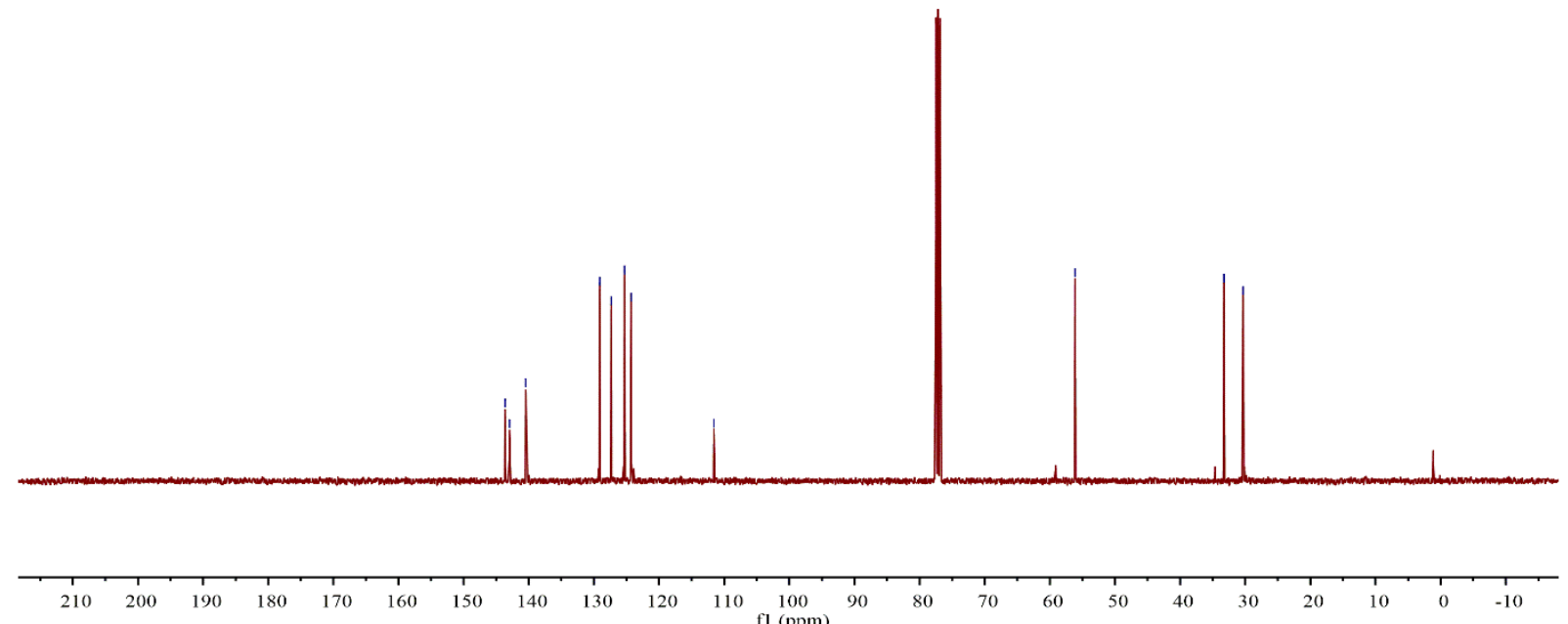

${ }^{13} \mathrm{C}$ NMR Spectrum of Compound $\mathbf{3 u}\left(100 \mathrm{MHz} \mathrm{CDCl}_{3}\right.$ )
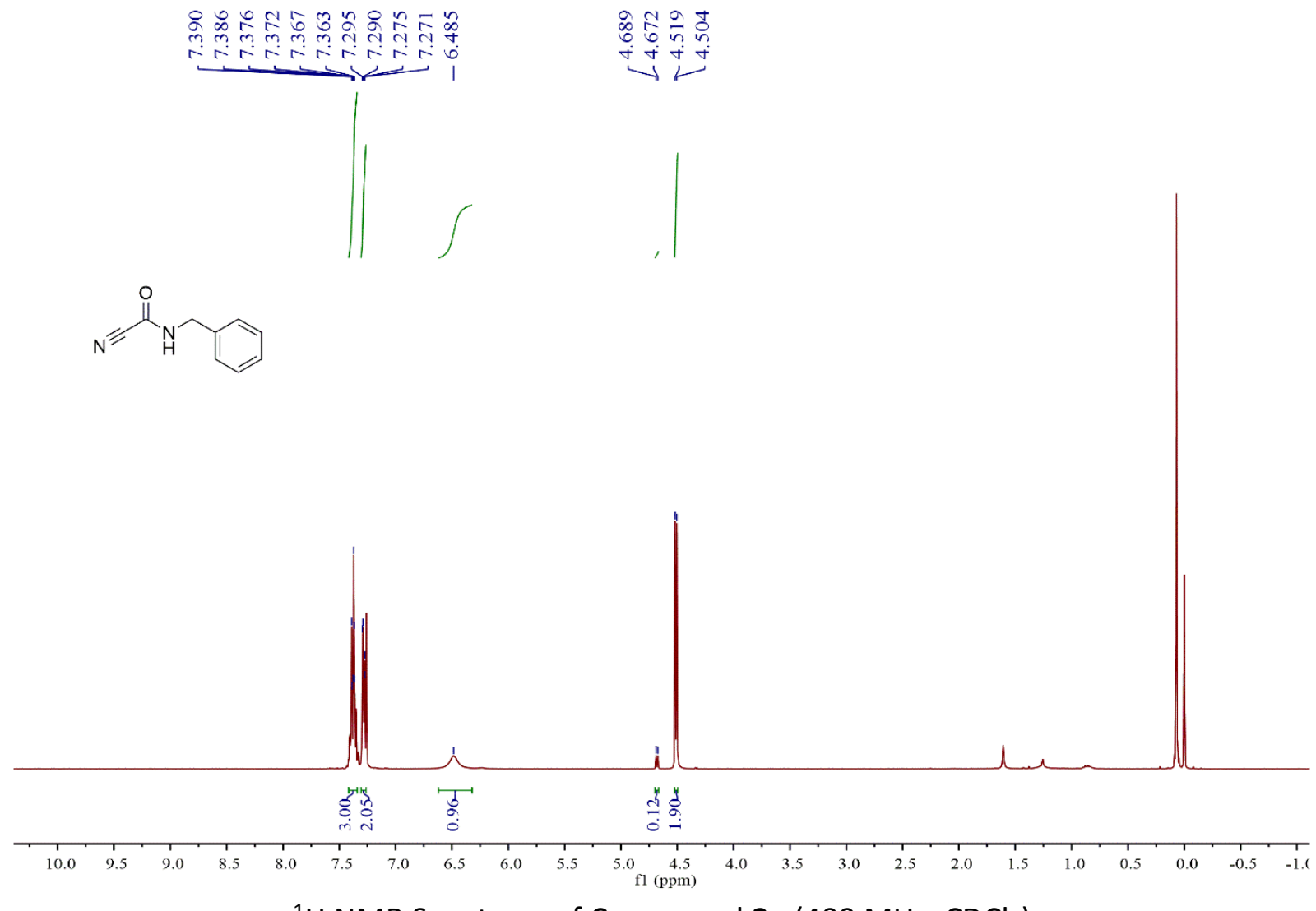

${ }^{1} \mathrm{H}$ NMR Spectrum of Compound $3 \mathbf{v}\left(400 \mathrm{MHz}, \mathrm{CDCl}_{3}\right)$ 

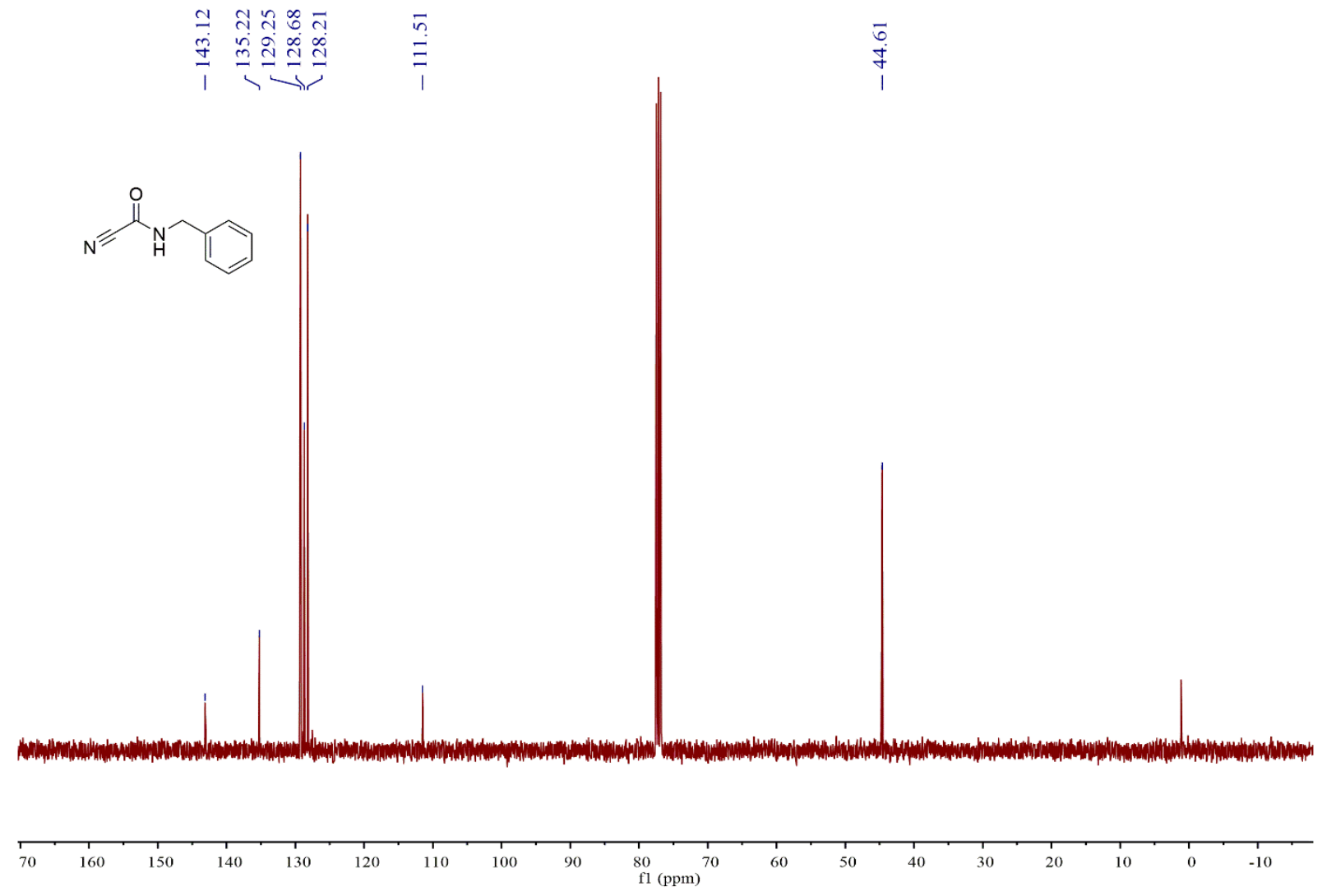

${ }^{13} \mathrm{C}$ NMR Spectrum of Compound $3 \mathbf{v}\left(100 \mathrm{MHz} \mathrm{CDCl}_{3}\right)$

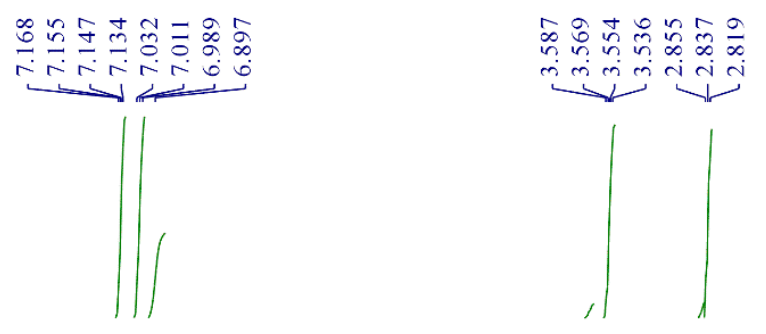

$N=\mathrm{N}^{\mathrm{H}}$

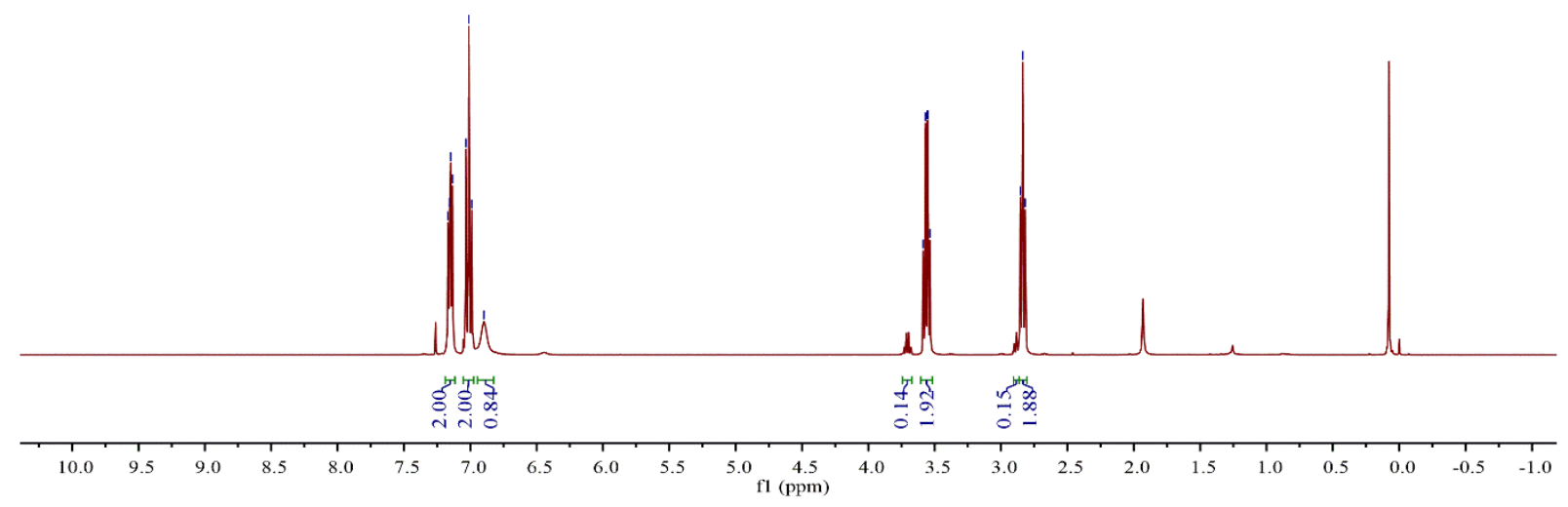

${ }^{1} \mathrm{H}$ NMR Spectrum of Compound $3 \mathbf{w}\left(400 \mathrm{MHz}, \mathrm{CDCl}_{3}\right)$ 

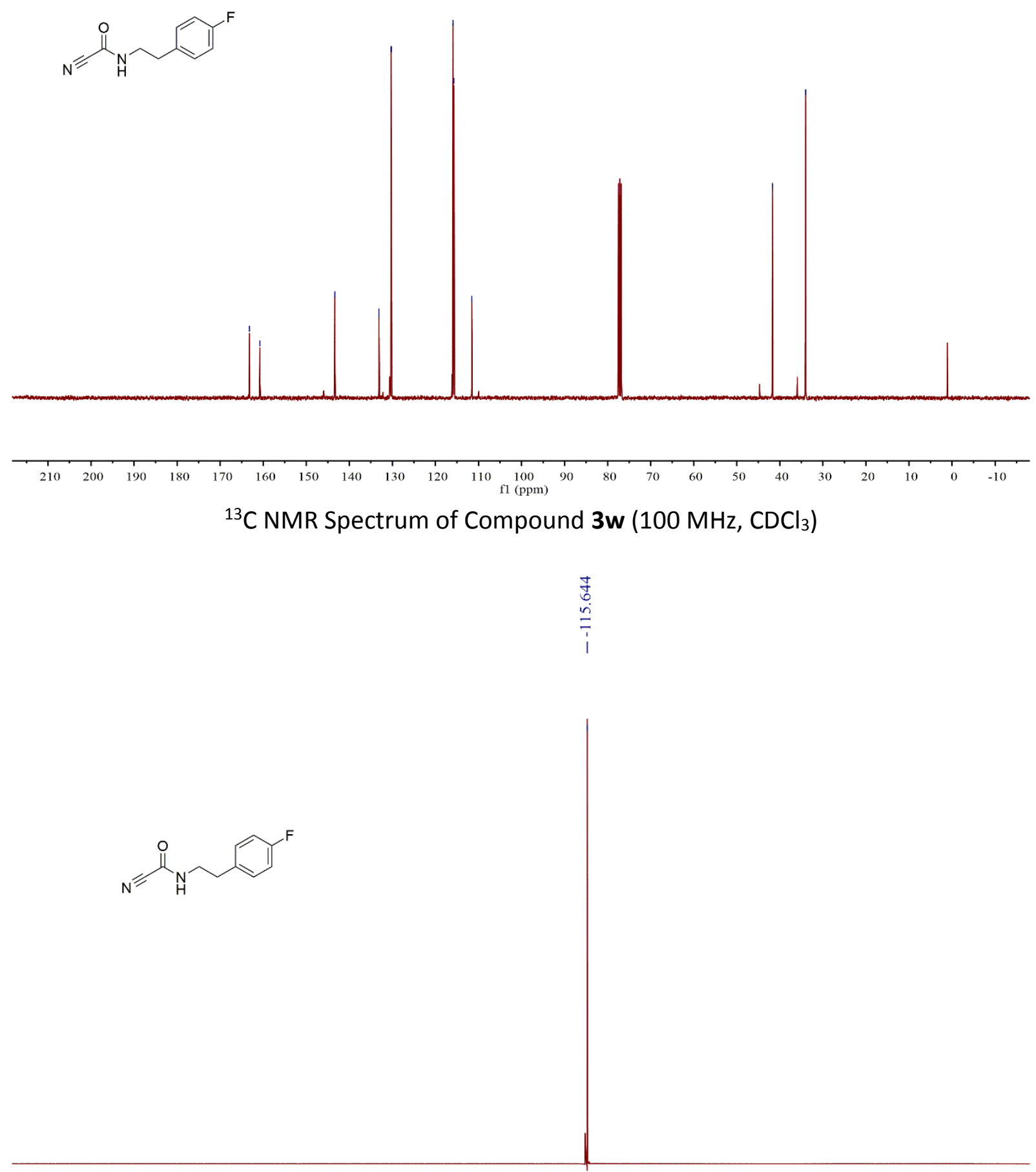

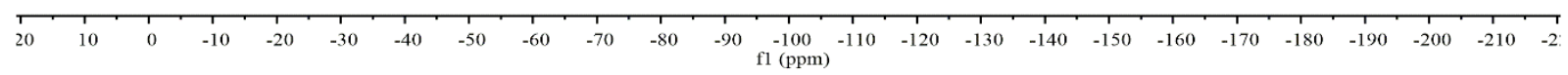

${ }^{19} \mathrm{~F}$ NMR Spectrum of Compound $3 \mathbf{w}\left(376 \mathrm{MHz}^{\mathrm{CDCl}} \mathrm{CD}_{3}\right)$ 

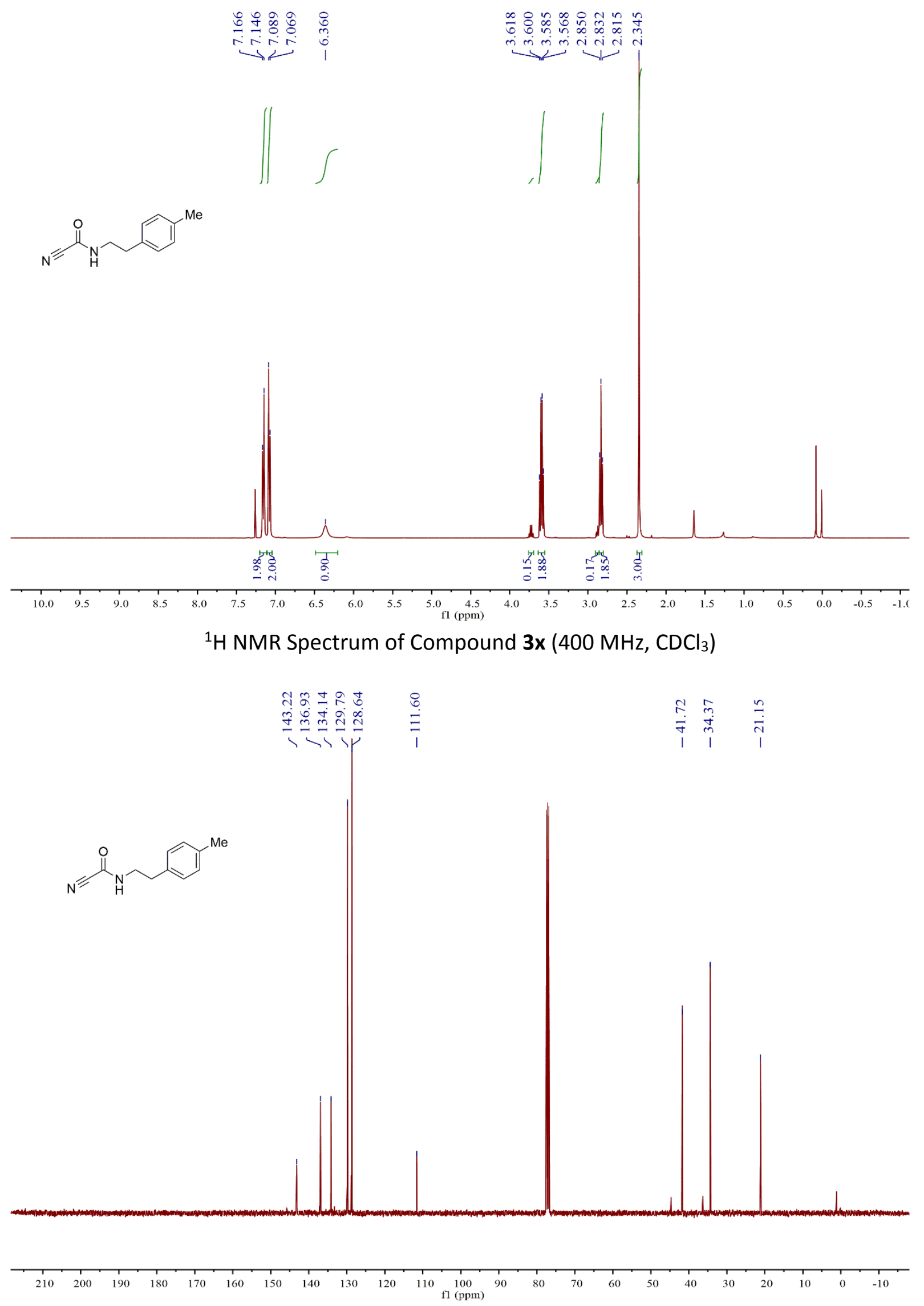

${ }^{13} \mathrm{C}$ NMR Spectrum of Compound $\mathbf{3 x}\left(100 \mathrm{MHz}, \mathrm{CDCl}_{3}\right)$ 

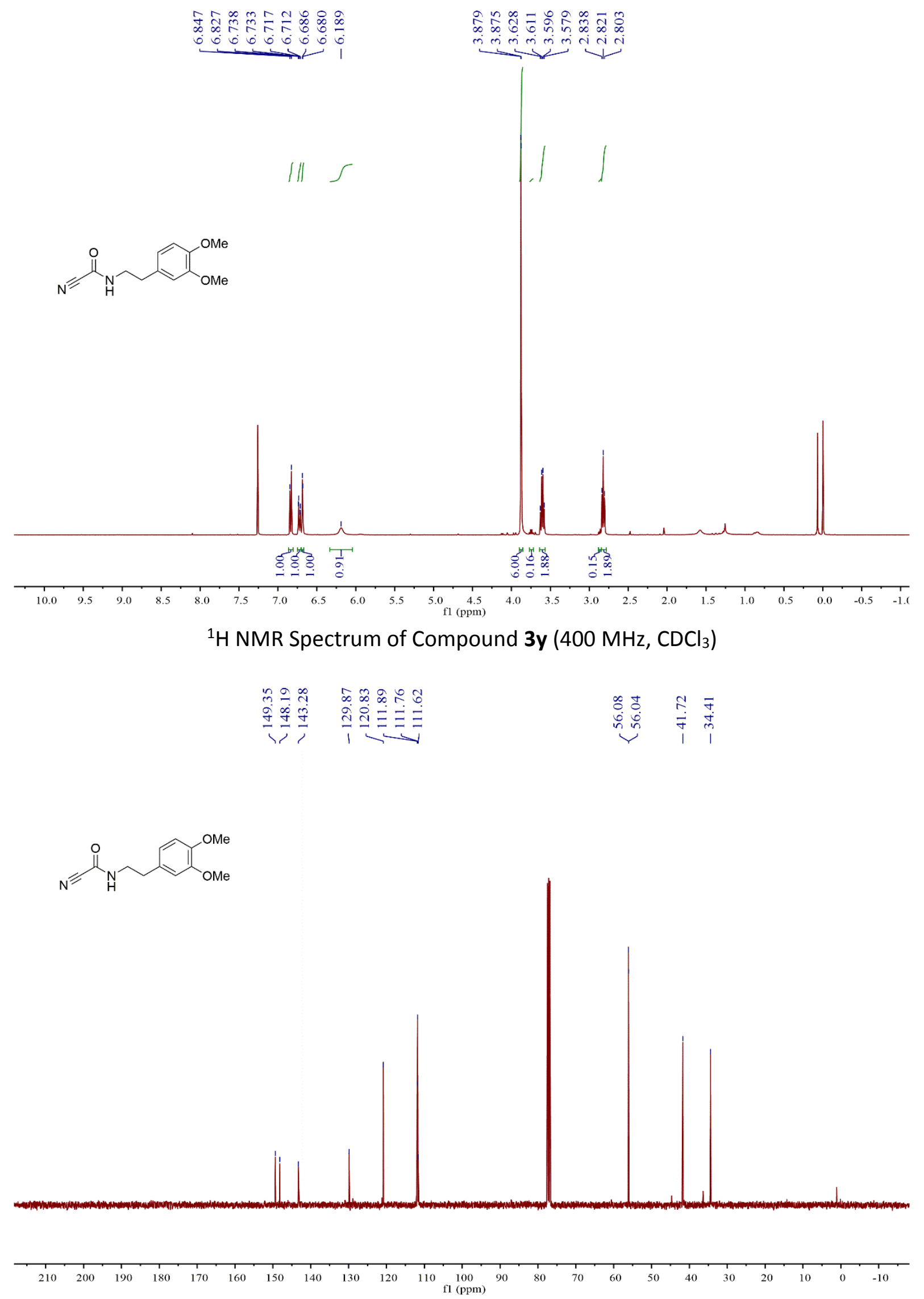

${ }^{13} \mathrm{C}$ NMR Spectrum of Compound $3 y\left(100 \mathrm{MHz}, \mathrm{CDCl}_{3}\right)$ 


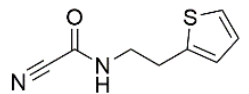
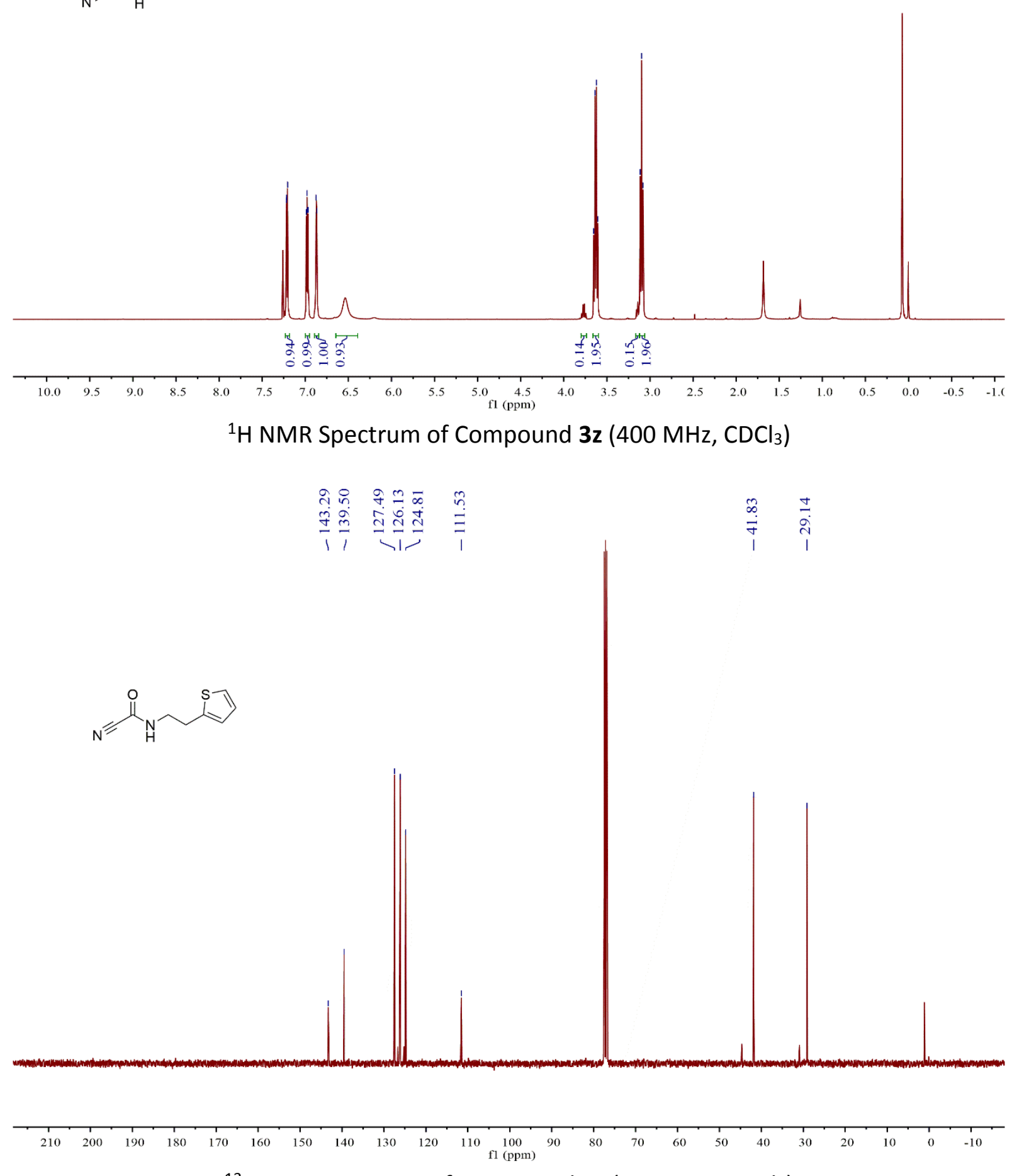

${ }^{13} \mathrm{C}$ NMR Spectrum of Compound $3 z\left(100 \mathrm{MHz}, \mathrm{CDCl}_{3}\right)$ 
<smiles>NC(=O)NCCCc1ccccc1</smiles>

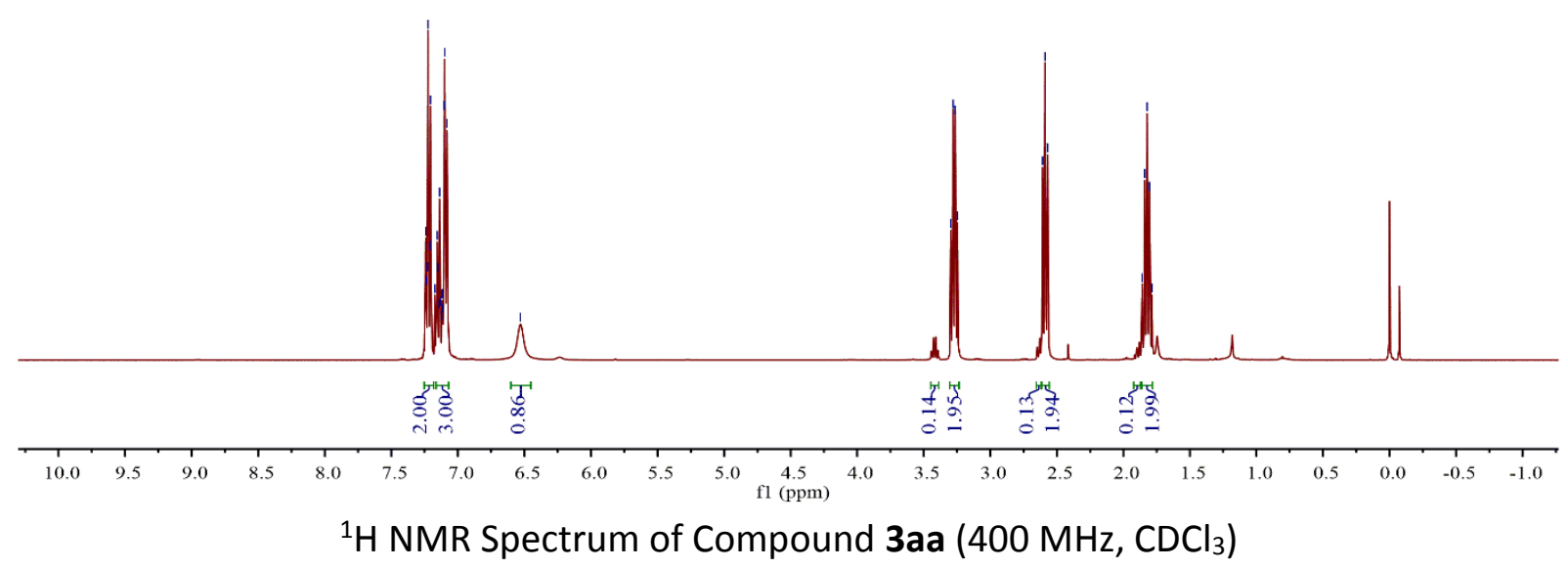<smiles>N#CC(=O)NCCCc1ccccc1</smiles>
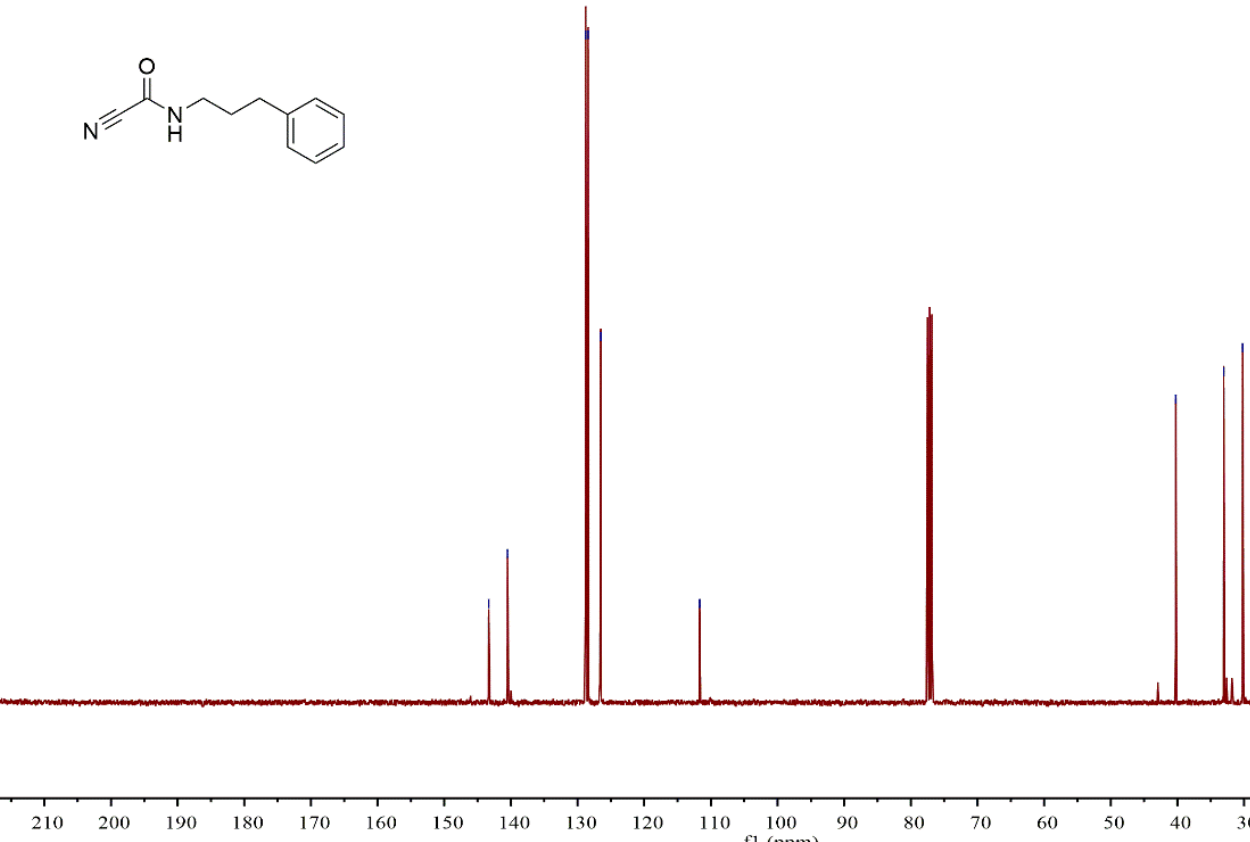

${ }^{13} \mathrm{C}$ NMR Spectrum of Compound 3aa $\left(100 \mathrm{MHz}, \mathrm{CDCl}_{3}\right)$ 


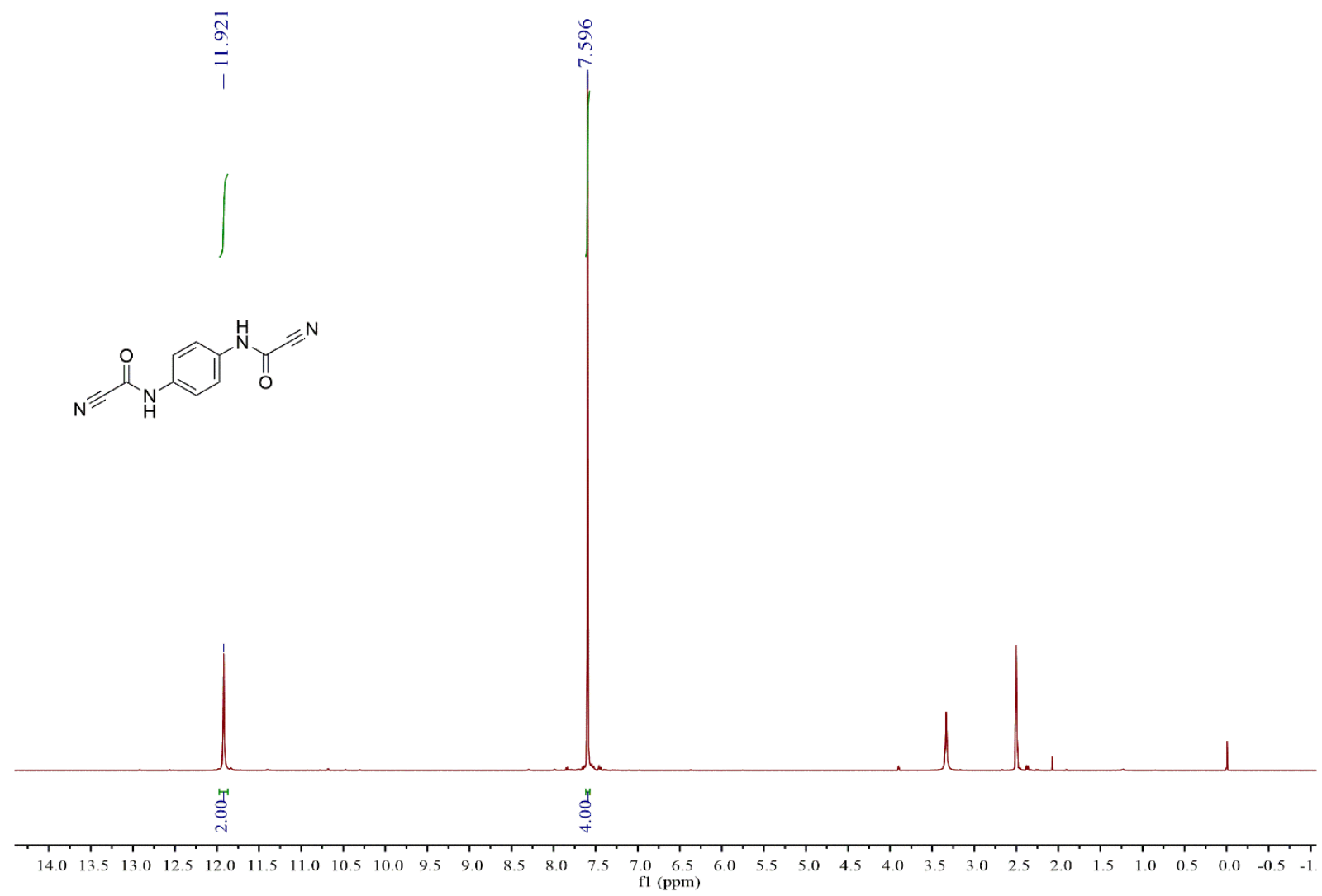

${ }^{1} \mathrm{H}$ NMR Spectrum of Compound $3 \mathrm{ab}\left(400 \mathrm{MHz},\left(\mathrm{CD}_{3}\right)_{2} \mathrm{SO}\right)$

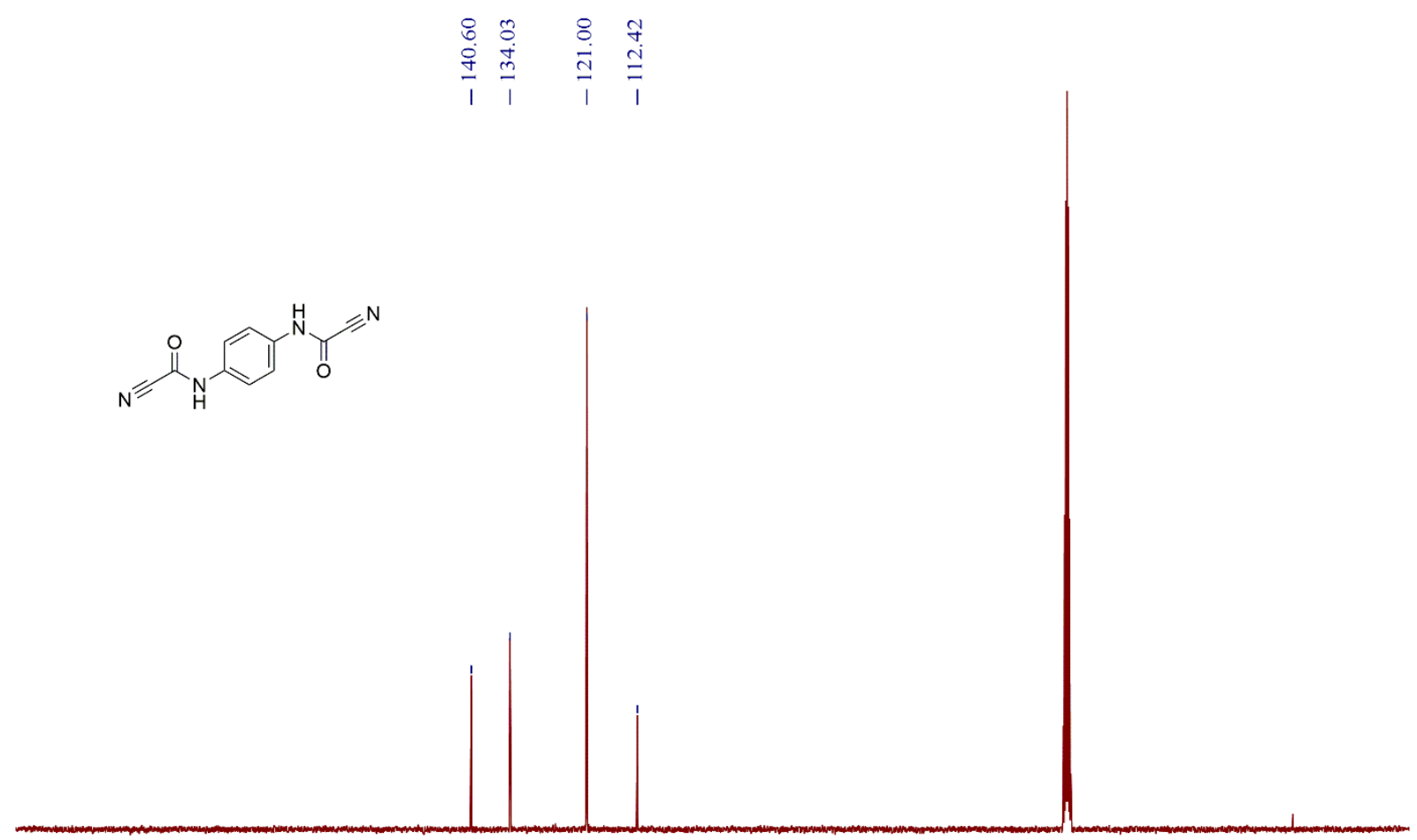

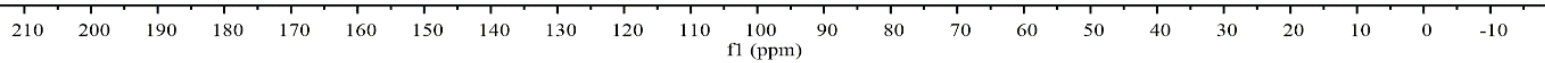

${ }^{13} \mathrm{C}$ NMR Spectrum of Compound $3 \mathrm{ab}\left(100 \mathrm{MHz},\left(\mathrm{CD}_{3}\right)_{2} \mathrm{SO}\right)$ 


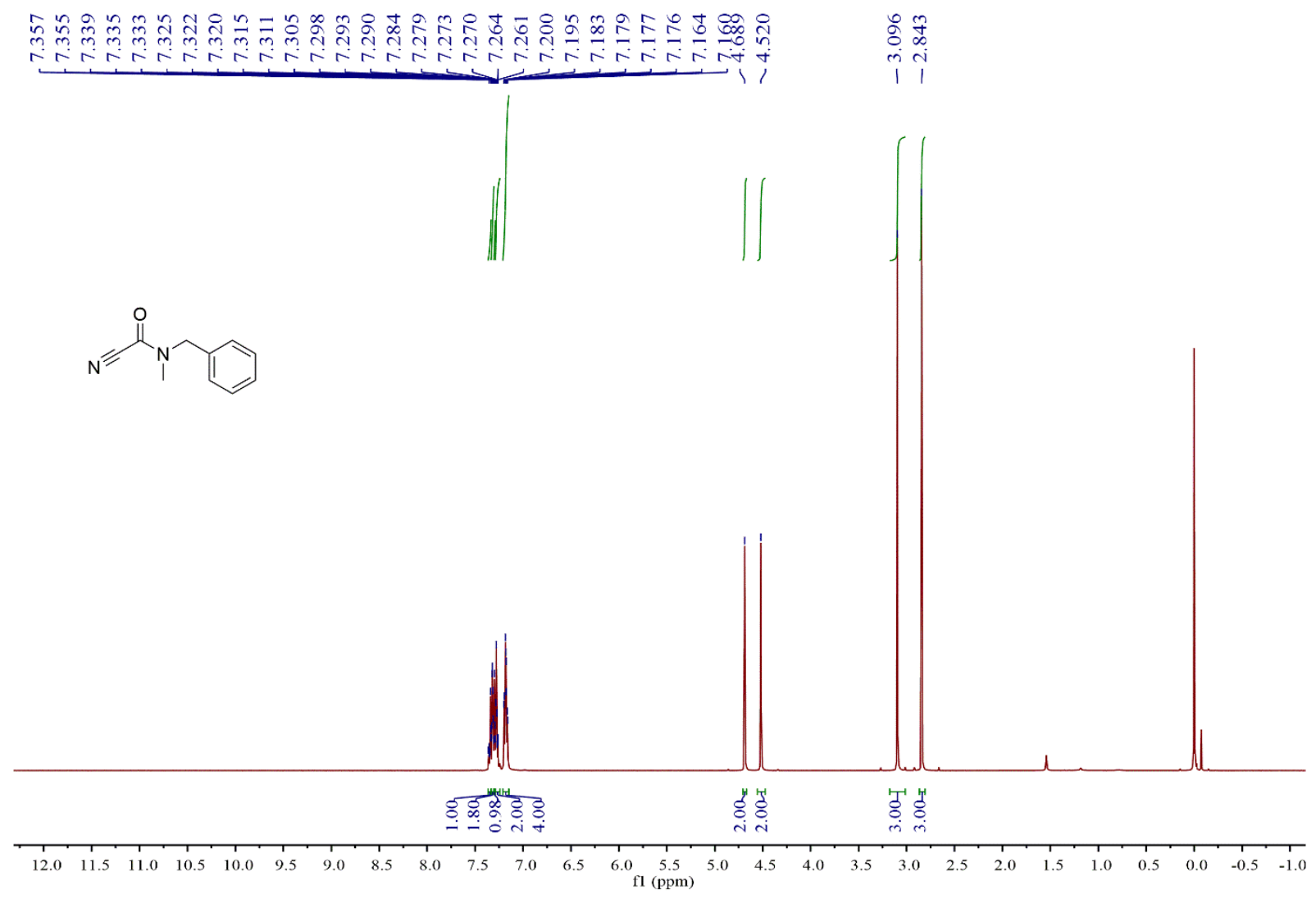

${ }^{1} \mathrm{H}$ NMR Spectrum of Compound $3 \mathrm{ac}\left(400 \mathrm{MHz}, \mathrm{CDCl}_{3}\right)$
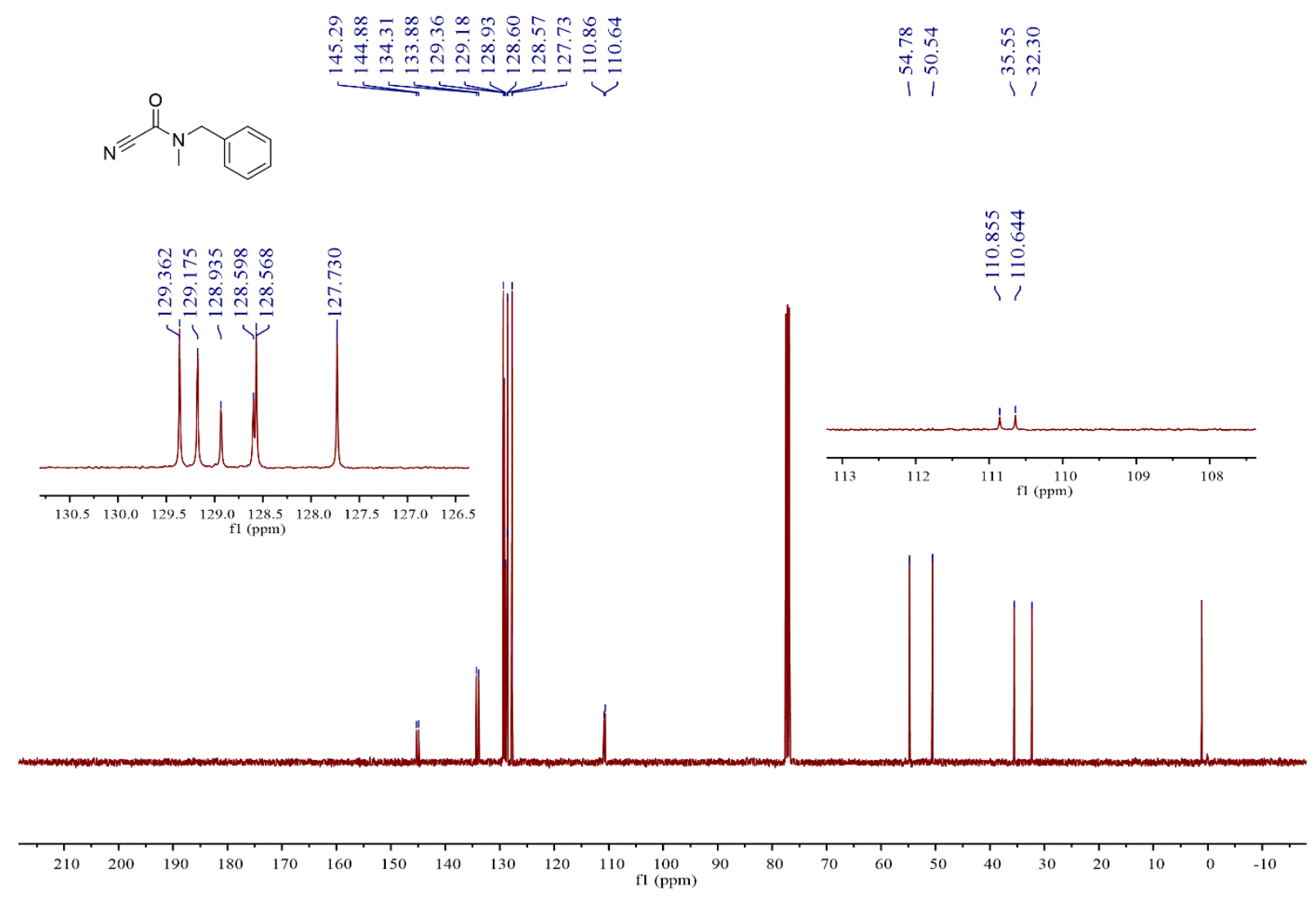

${ }^{13} \mathrm{C}$ NMR Spectrum of Compound $3 \mathrm{ac}\left(100 \mathrm{MHz}, \mathrm{CDCl}_{3}\right)$ 

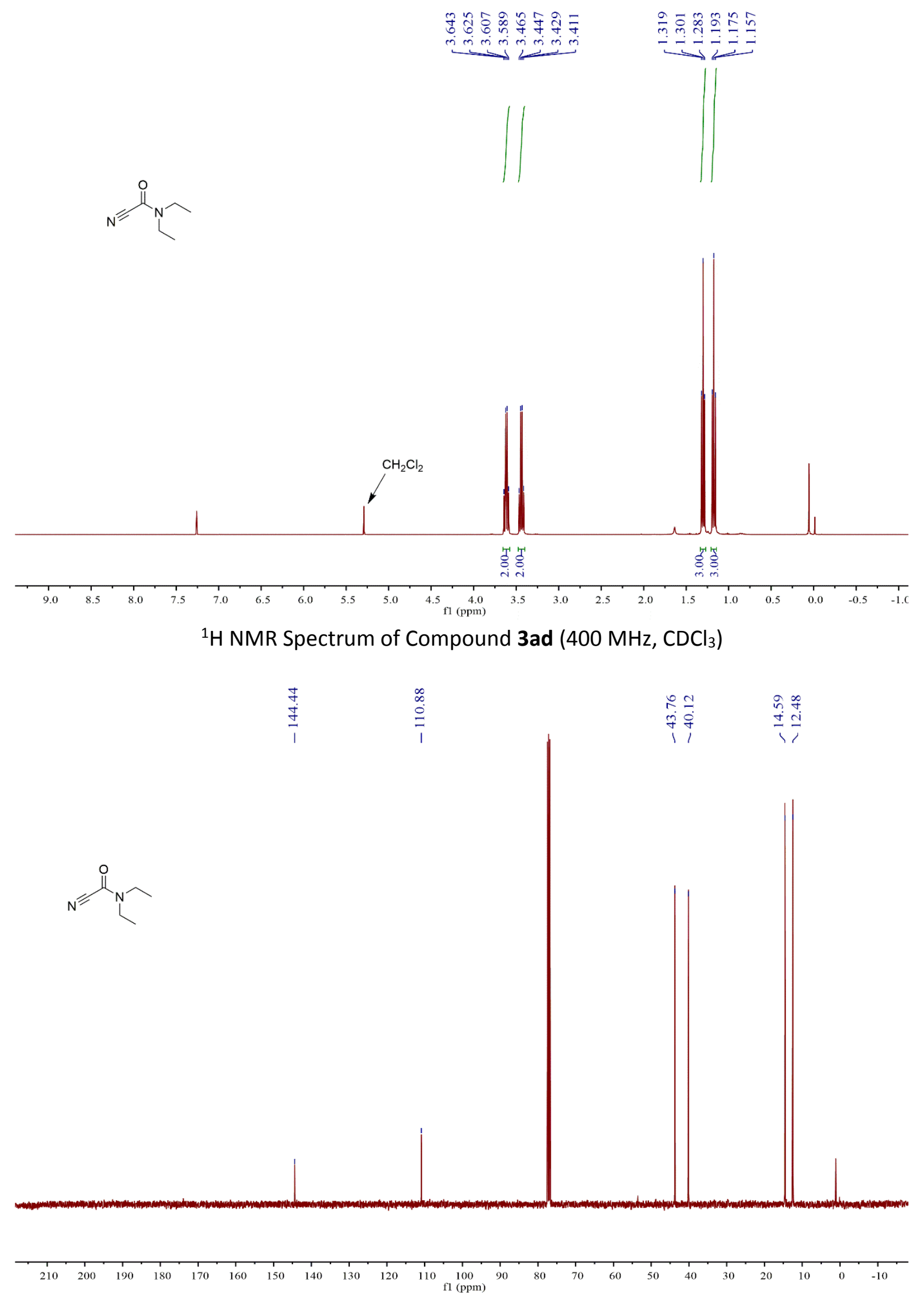

${ }^{13} \mathrm{C}$ NMR Spectrum of Compound $3 a d\left(100 \mathrm{MHz}, \mathrm{CDCl}_{3}\right.$ ) 

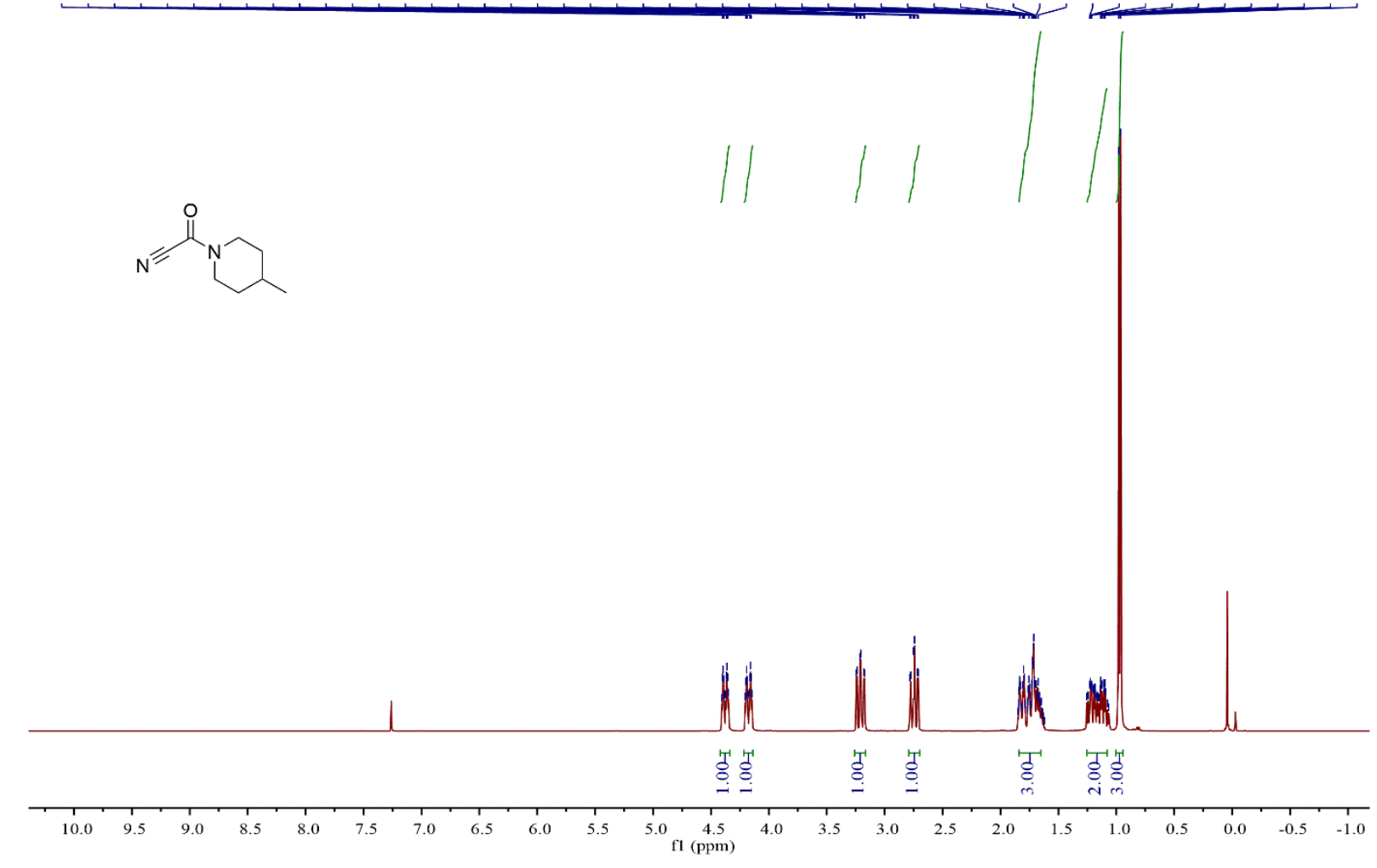

${ }^{1} \mathrm{H}$ NMR Spectrum of Compound 3ae $\left(400 \mathrm{MHz}^{\mathrm{CDCl}}{ }_{3}\right)$
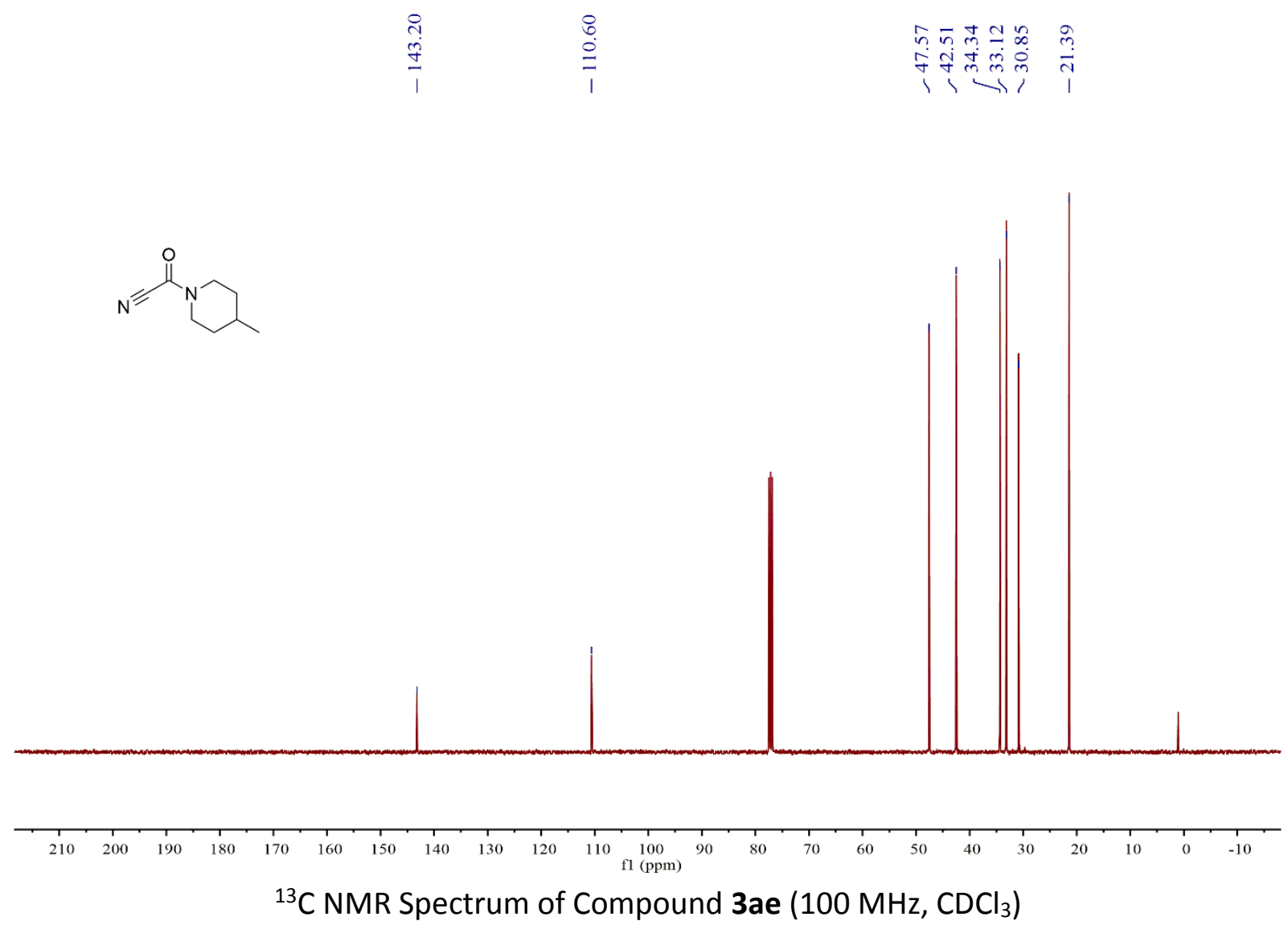


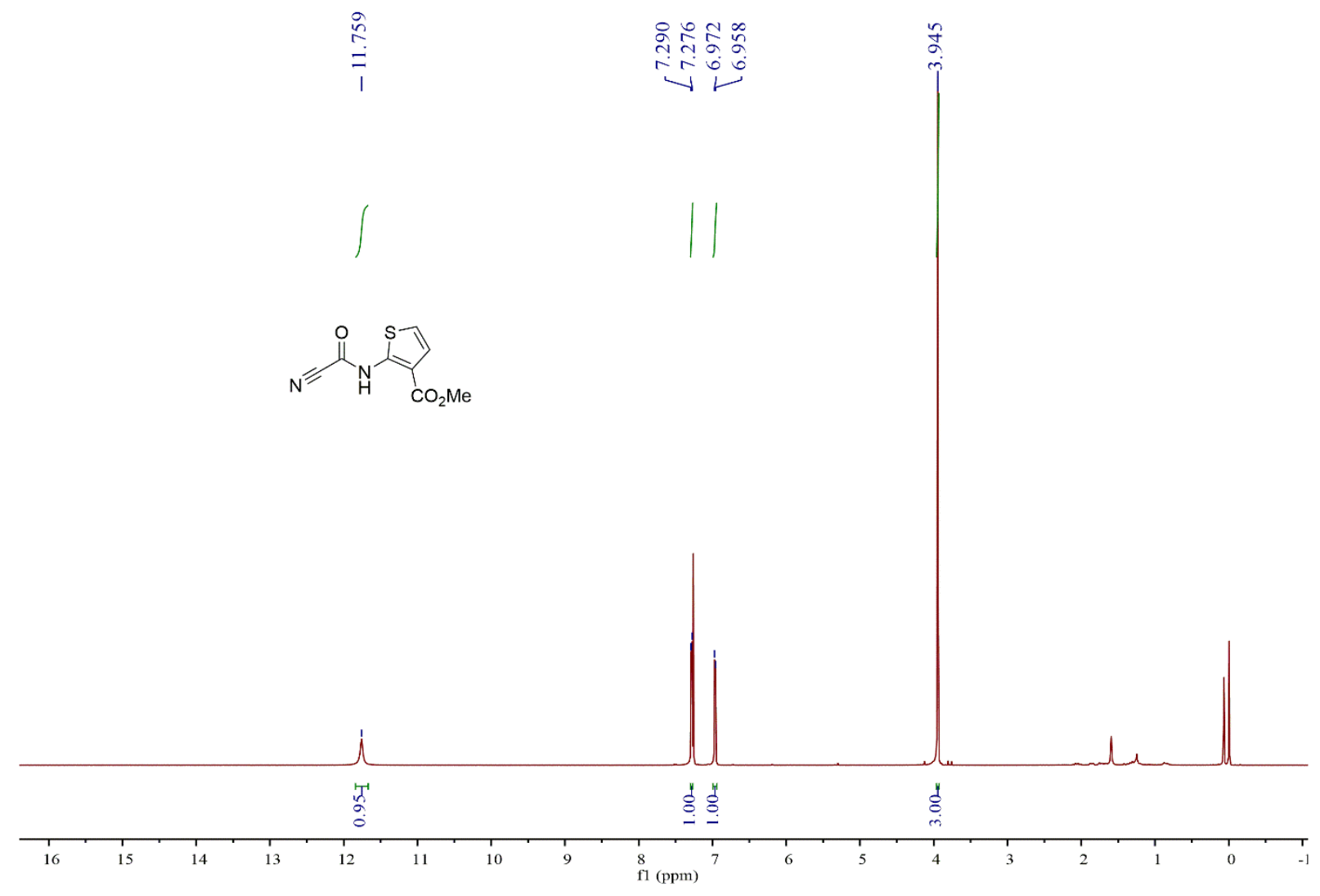

${ }^{1} \mathrm{H}$ NMR Spectrum of Compound 3 af $\left(400 \mathrm{MHz}, \mathrm{CDCl}_{3}\right)$

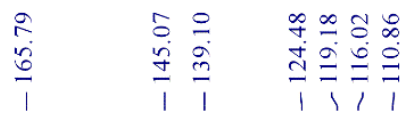
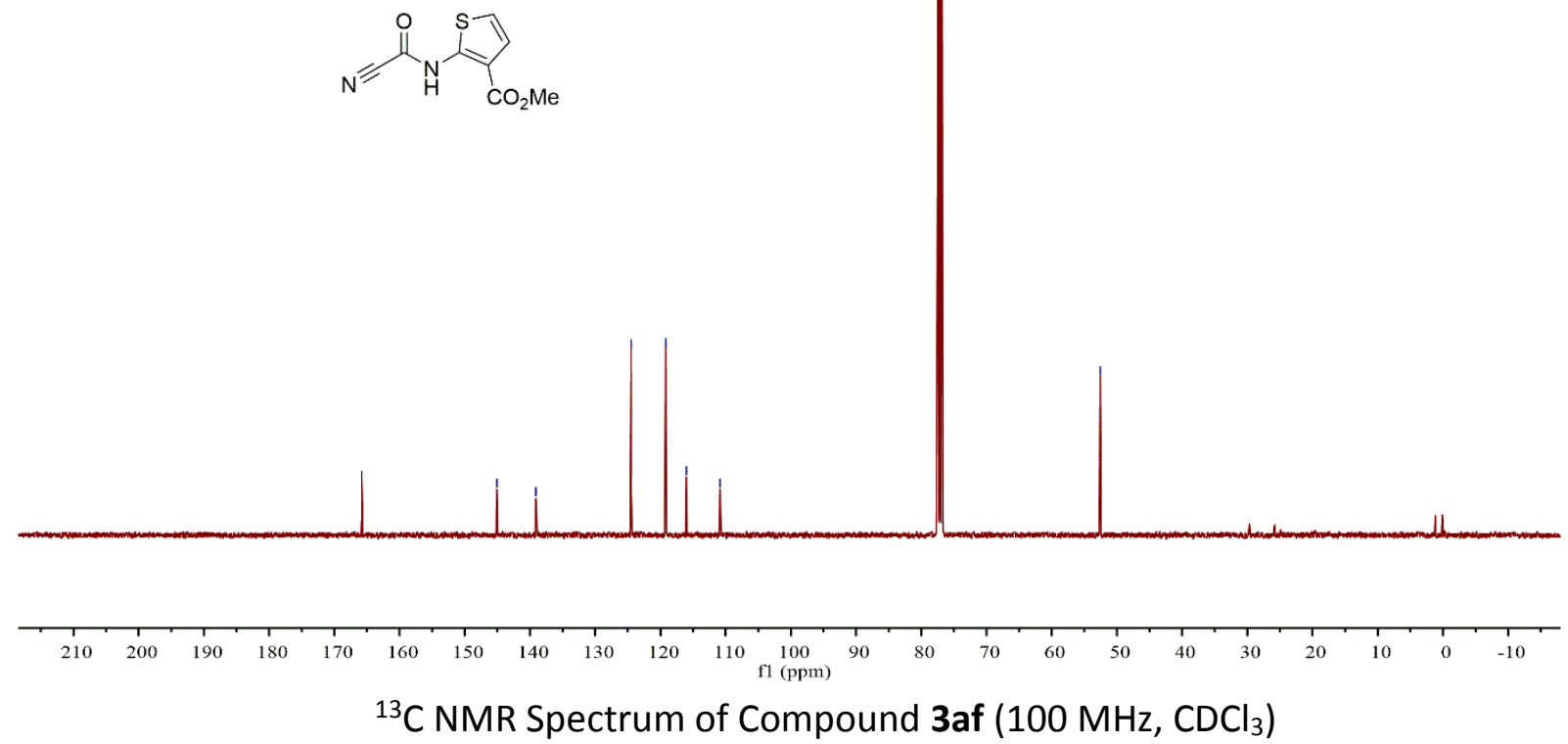

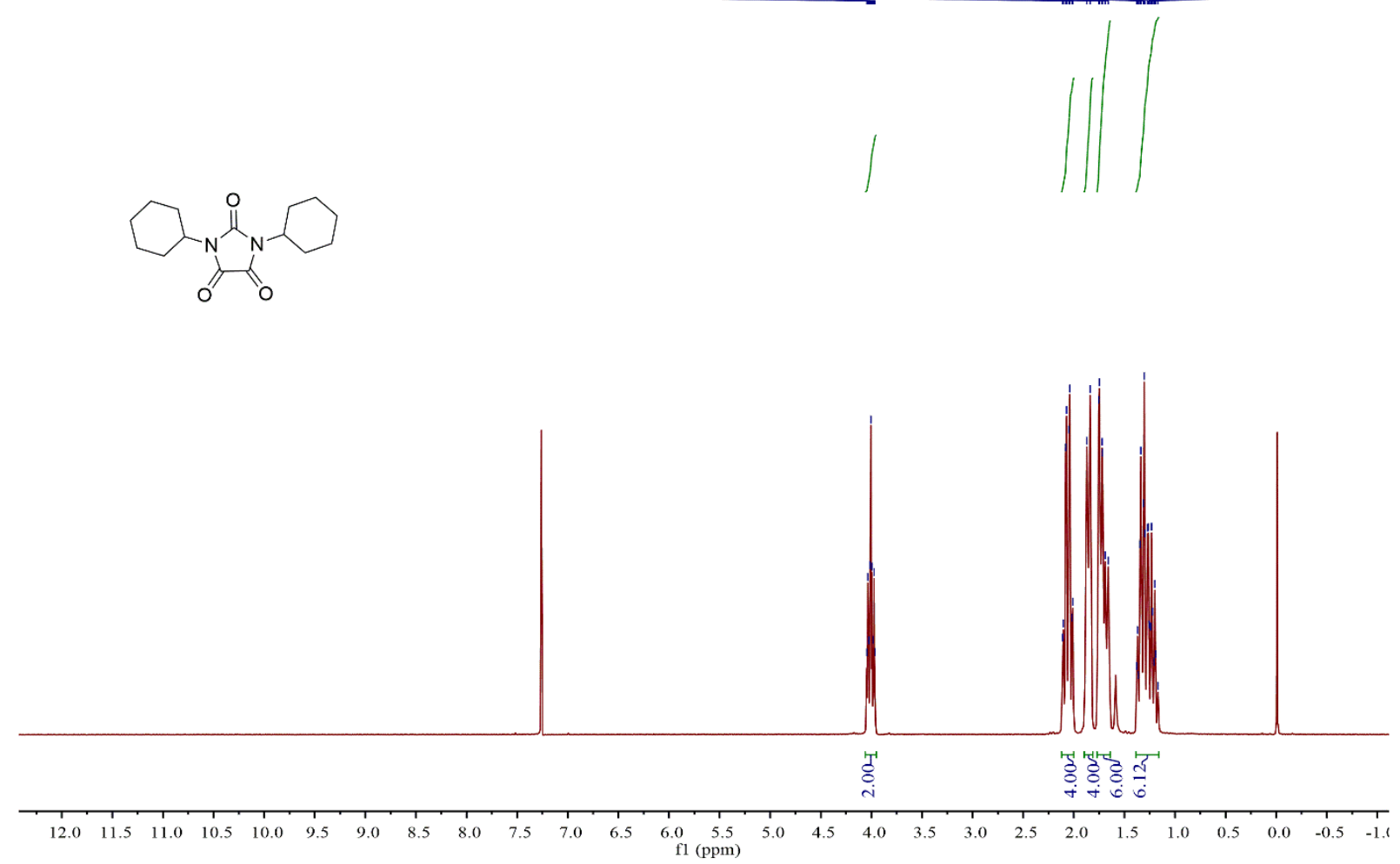

${ }^{1} \mathrm{H}$ NMR Spectrum of Compound $4\left(400 \mathrm{MHz}, \mathrm{CDCl}_{3}\right)$
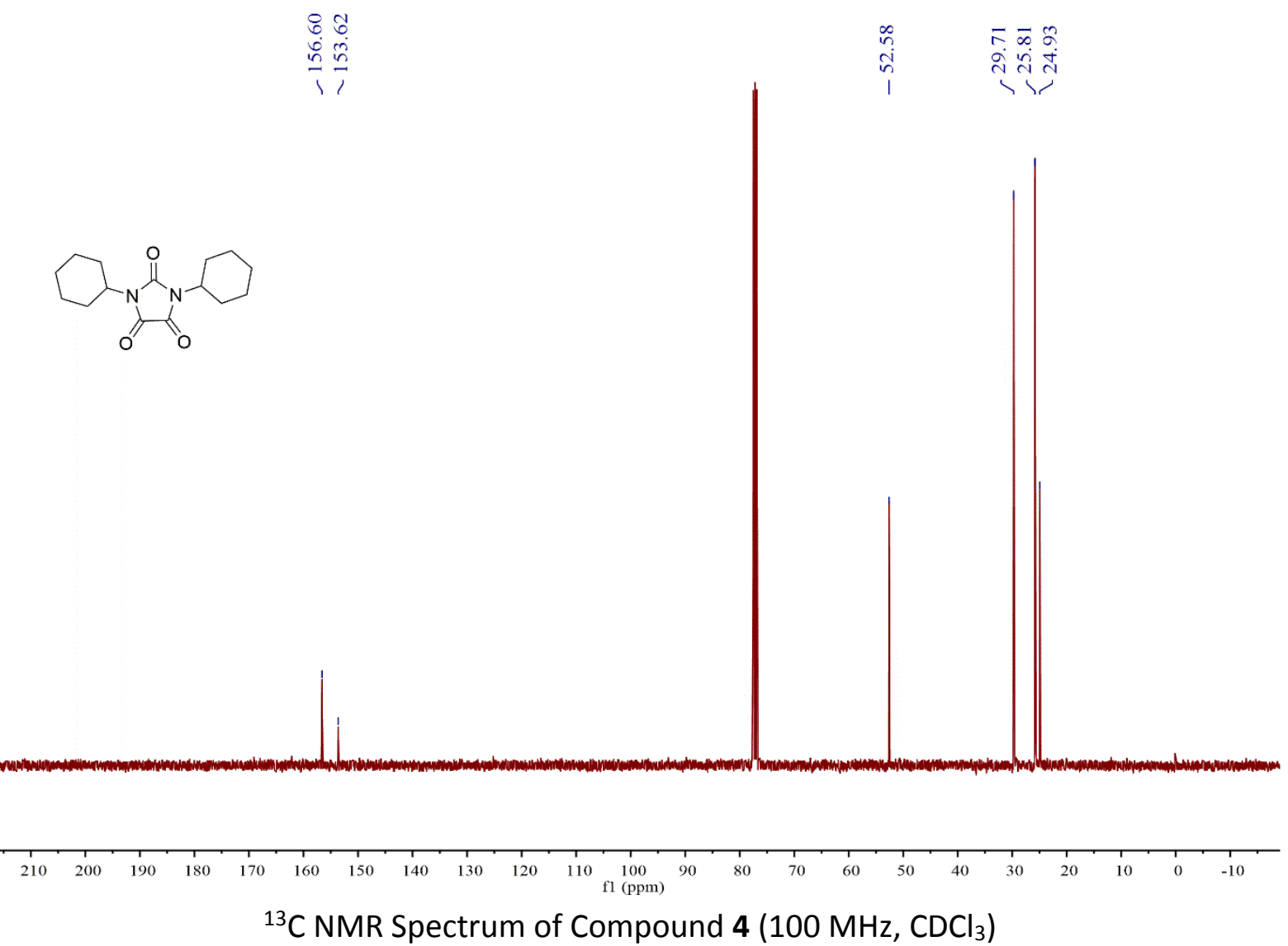
<smiles>CC(C)(C)OC(=O)NCC(=O)Nc1ccccc1</smiles>

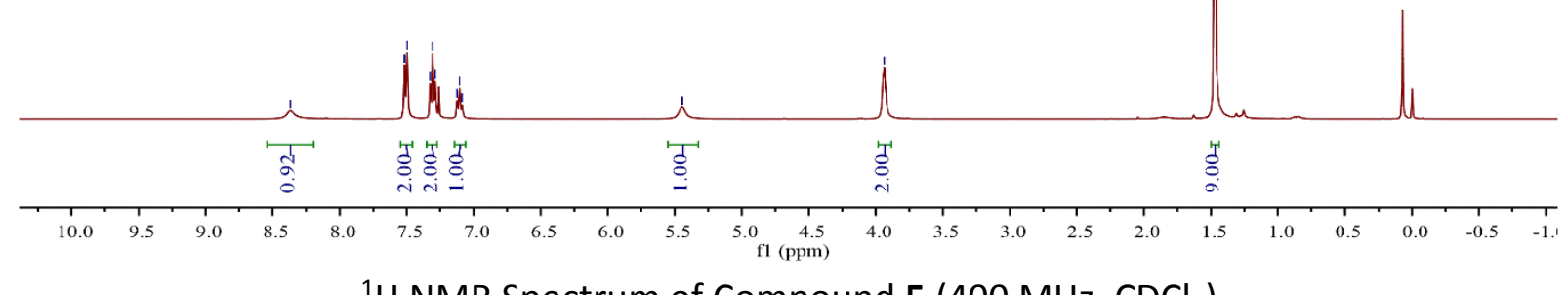

${ }^{1} \mathrm{H}$ NMR Spectrum of Compound $5\left(400 \mathrm{MHz} \mathrm{CDCl}_{3}\right)$

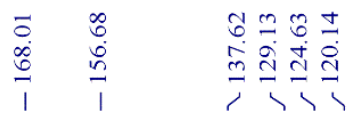

$>\prod^{O}{ }_{H}^{H}$
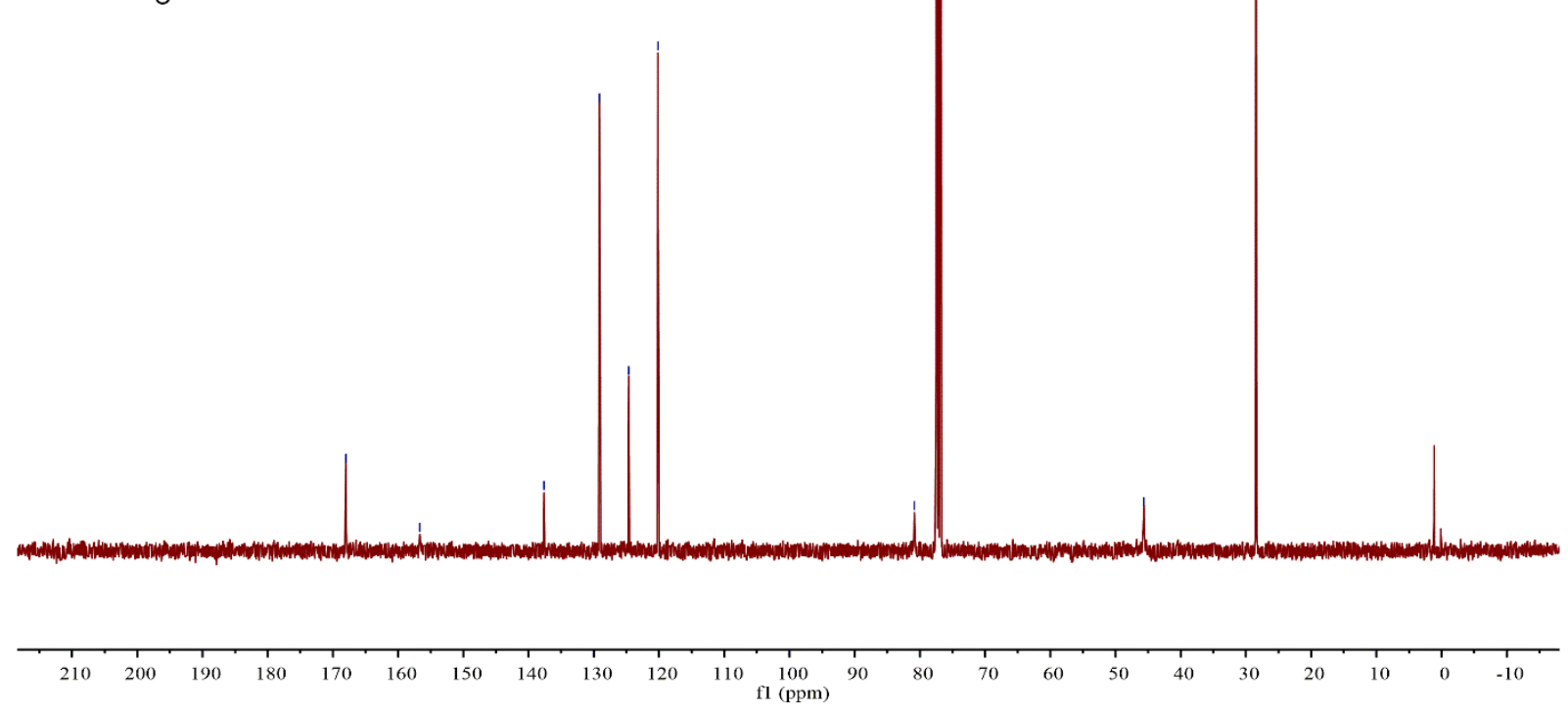

${ }^{13} \mathrm{C}$ NMR Spectrum of Compound $5\left(100 \mathrm{MHz}, \mathrm{CDCl}_{3}\right)$ 

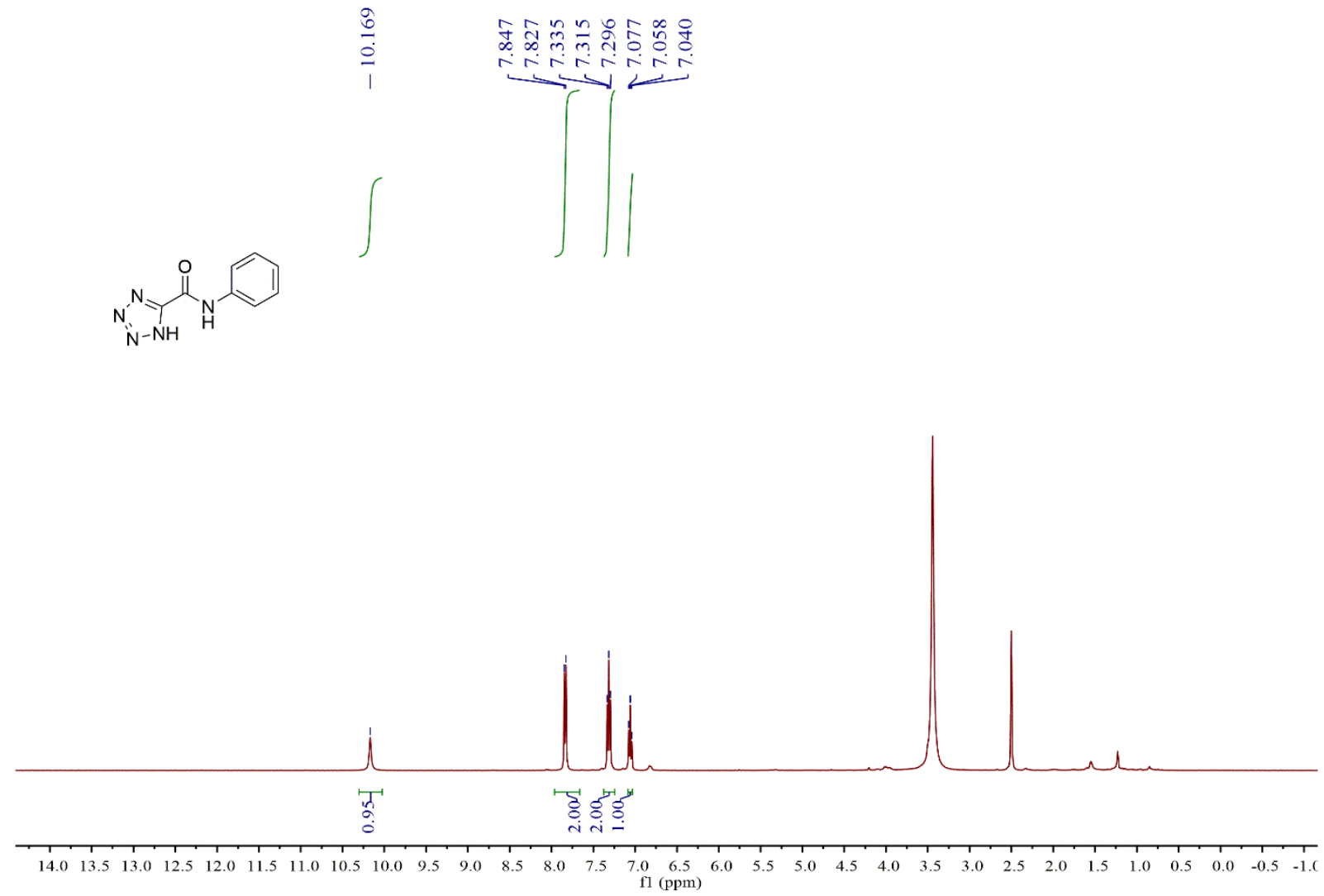

${ }^{1} \mathrm{H}$ NMR Spectrum of Compound $6\left(400 \mathrm{MHz},\left(\mathrm{CD}_{3}\right)_{2} \mathrm{SO}\right)$
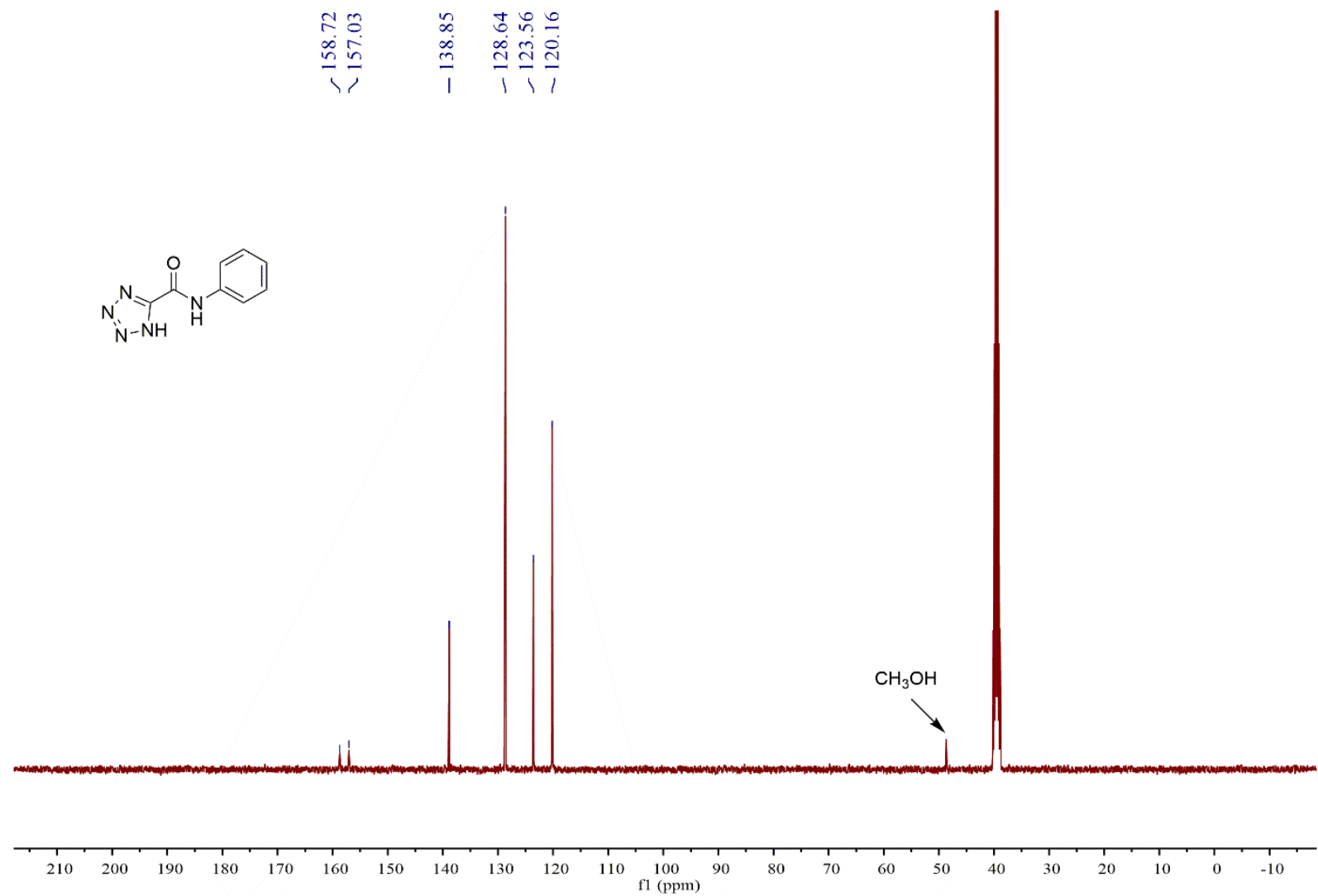

${ }^{13} \mathrm{C}$ NMR Spectrum of Compound $6\left(100 \mathrm{MHz},\left(\mathrm{CD}_{3}\right)_{2} \mathrm{SO}\right)$ 

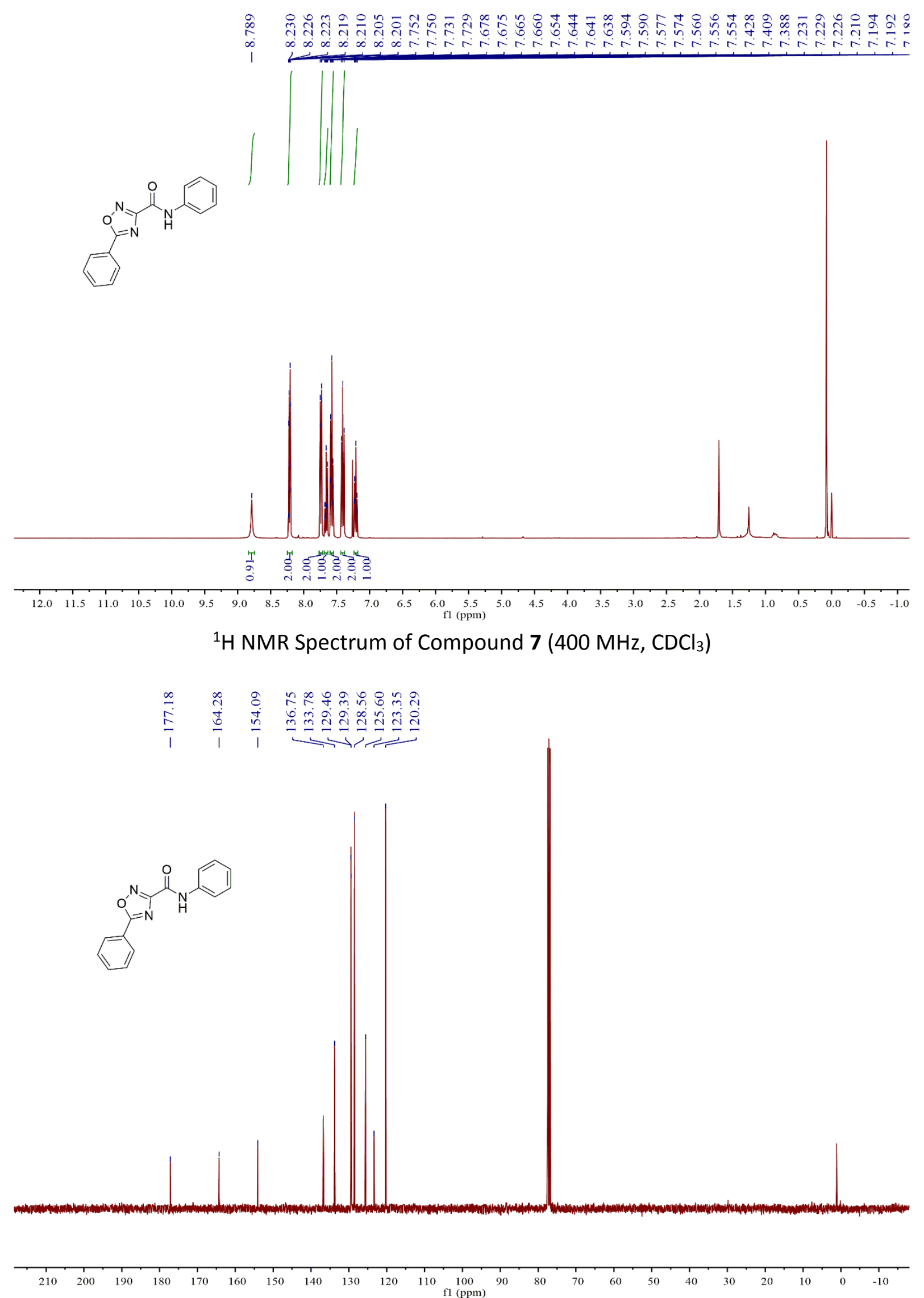

${ }^{13} \mathrm{C}$ NMR Spectrum of Compound $7\left(100 \mathrm{MHz}, \mathrm{CDCl}_{3}\right)$ 


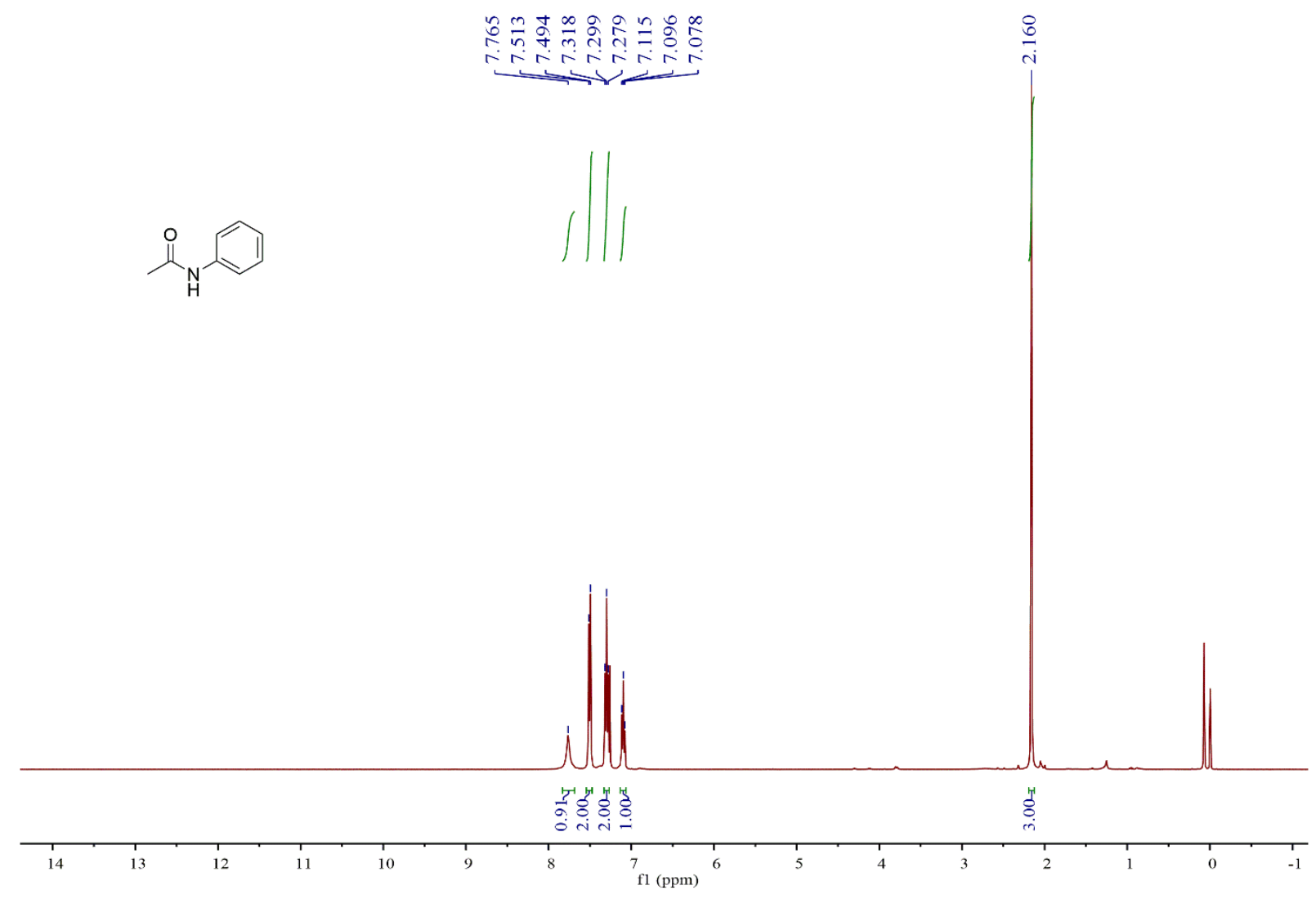

${ }^{1} \mathrm{H}$ NMR Spectrum of $\boldsymbol{N}$-Phenylacetamide $\left(400 \mathrm{MHz}^{-\mathrm{CDCl}_{3}}\right)$
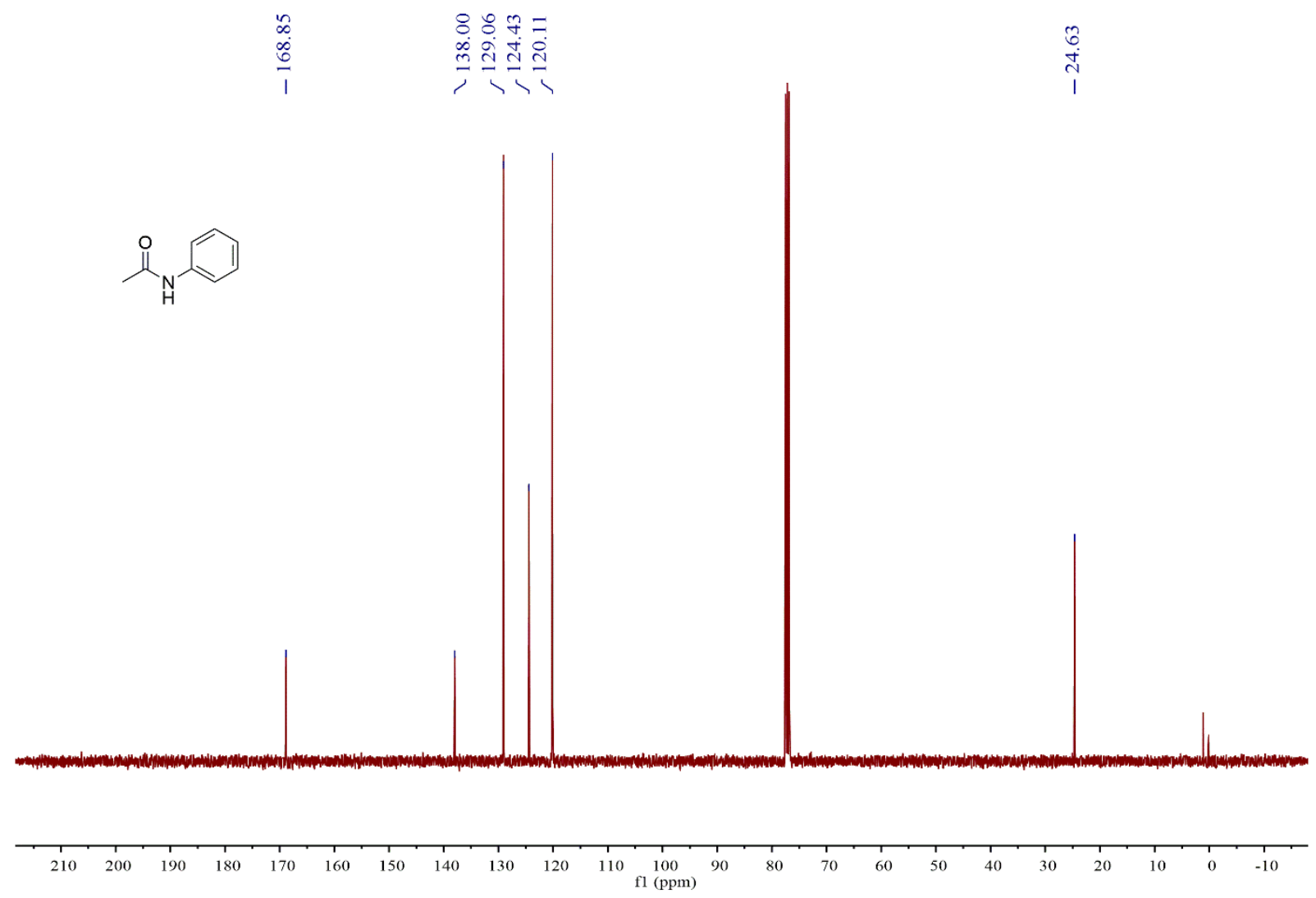

${ }^{13} \mathrm{C}$ NMR Spectrum of $\boldsymbol{N}$-Phenylacetamide $\left(100 \mathrm{MHz}, \mathrm{CDCl}_{3}\right)$ 